\title{
WestVirginiaUniversity
}

THE RESEARCH REPOSITORY @ WVU

Graduate Theses, Dissertations, and Problem Reports

2004

\section{Silicon carbide epitaxial growth using methylsilanes as gas sources}

Charlie Y. Peng

West Virginia University

Follow this and additional works at: https://researchrepository.wvu.edu/etd

\section{Recommended Citation}

Peng, Charlie Y., "Silicon carbide epitaxial growth using methylsilanes as gas sources" (2004). Graduate Theses, Dissertations, and Problem Reports. 2609.

https://researchrepository.wvu.edu/etd/2609

This Dissertation is protected by copyright and/or related rights. It has been brought to you by the The Research Repository @ WVU with permission from the rights-holder(s). You are free to use this Dissertation in any way that is permitted by the copyright and related rights legislation that applies to your use. For other uses you must obtain permission from the rights-holder(s) directly, unless additional rights are indicated by a Creative Commons license in the record and/ or on the work itself. This Dissertation has been accepted for inclusion in WVU Graduate Theses, Dissertations, and Problem Reports collection by an authorized administrator of The Research Repository @ WVU.

For more information, please contact researchrepository@mail.wvu.edu. 


\title{
Silicon Carbide Epitaxial Growth Using Methylsilanes as Gas Sources
}

\author{
Charlie Y. Peng
}

\author{
Dissertation Submitted to the \\ College of Engineering and Mineral Resources \\ at West Virginia University \\ in partial fulfillment of the requirements \\ for the degree of
}

Doctor of Philosophy

in

Chemical Engineering

\author{
Charter Stinespring, Ph.D., Chair \\ Mark Jerabek, Ph.D. \\ Edwin Kugler, Ph. D. \\ Thomas Myers, Ph.D. \\ John Zondlo, Ph.D. \\ Department of Chemical Engineering
}

Morgantown, West Virginia

2004

Keywords: $6 \mathrm{H}-\mathrm{SiC}$, thin film, homoepitaxy, heteroepitaxy, high temperature hydrogen etch, MS, DMS

Copyright 2004 Charlie Y. Peng 


\section{ABSTRACT \\ Silicon Carbide Epitaxial Growth Using Methylsilanes as Gas Sources}

Charlie Y. Peng

Large area and high quality $\mathrm{SiC}$ substrates are required for many applications. The goal of this research is to develop novel methods of growing epitaxial silicon carbide ( $\mathrm{SiC}$ ) on $6 \mathrm{H}-$ $\mathrm{SiC}$ and silicon ( $\mathrm{Si}$ ) substrates while extending our understanding of the growth mechanisms and the effects of key growth parameters. High temperature hydrogen-etching procedures for preparing atomically-stepped $6 \mathrm{H}-\mathrm{SiC}$ substrates suitable for epitaxial growth were also developed.

This dissertation presents results of both homoepitaxial $\mathrm{SiC}$ growth on $6 \mathrm{H}-\mathrm{SiC}$ substrates and heteroepitaxial growth on $\mathrm{Si}$ substrates by gas source molecular beam epitaxy. The experimental variables included gas species, molecular flux, growth time, and substrates growth temperature. In particular, the growth species considered here were methylsilane and dimethylsilane, and the substrate temperatures were $700^{\circ} \mathrm{C}$ and $800^{\circ} \mathrm{C}$. The thin films grown in these studies were characterized by Auger electron spectroscopy, reflection high Energy electron diffraction, field emission scanning electron microscopy, and atomic force microscopy.

Homoepitaxial films grown on $6 \mathrm{H}-\mathrm{SiC}$ substrates showed little to no change in surface chemical composition, surface crystal structure, and RMS roughness. Compared to the substrates, reductions in step height were detected consistent with previous observations for chemical vapor desposition of $\mathrm{SiC}$. Thick heteroepitaxial films could be grown on $\mathrm{Si}$ using methylsilane as the gas source at $800^{\circ} \mathrm{C}$, but voids caused by $\mathrm{Si}$ out-diffusion from the substrate were a problem still. Dimethylsilane produced thick epitaxial films at $800^{\circ} \mathrm{C}$ without substrate voids. In this case, however, the surface was terminated by $\mathrm{C}-\mathrm{C}$ bonds. 


\section{DEDICATION}

To my family, most of whom, residing on the other side of the world, have been separated from me for too long. I hope I will live up to your expectations and unconditional love to me. 


\section{ACKNOWLEDGEMENT}

First, I want to thank my research advisor, Dr. Stinespring. I could not have asked for a better mentor than him for the most important years of my life.

I want to thank DARPA, ONR, and NASA collectively for funding of this research project.

I want to thank the Department of Chemical Engineering collectively for affording me, through the ups and the downs, this wonderful learning experience.

I want to thank the members of my research committee, Dr. Jerabek, Dr. Kugler, Dr. Myers, and Dr. Zondlo, all of whom went beyond the call of duty to help me through this.

I want to thank my lab mates, Katie Ziemer for showing me the ropes when I first came to WVU, Andy Woodworth for his AFM work and all those entertaining conversations, and everyone else who came through while I was here: Jeff, Prasanah, Mohammad, Joe, and Srik.

I want to thank LDCSEE and Kolin Brown for allowing me to use their lab facilities.

I want to thank Kyoungnae Lee for her AFM work.

I want to thank Dr. Zondlo, Dr. Stansberry, and Liviu Magean for usages of the microbalance, FTIR, and FESEM over the years.

I want to thank Linda Cox of CEMR, Linda Rogers and Bonnie Helmick for all the administrative work they put forth for me.

I want to thank Department of Chemical Engineering shop supervisor Jim Hall, for all his work with us and for machining those bizarre parts we always needed.

I want to thank all my friends here at WVU. I hope you have enjoyed the friendships as much as I have.

I want to thank Wendy, who is always there to encourage and comfort me when things appear bleak.

Last but certainly the most, I want to thank my mom, who worked so hard all of her life and gave me this great opportunity. Without her, none of this would be possible.

At this crossroads of my life, I want to quote Percy Bysshe Shelley, for what he said was always how I envision one's life should be lived:

"Fear not for the future, weep not for the past." 


\title{
Table of Contents
}

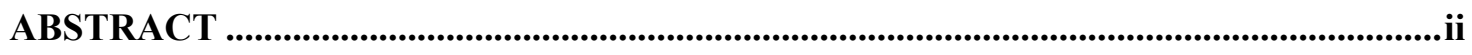

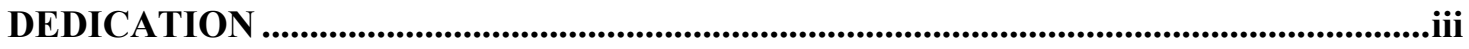

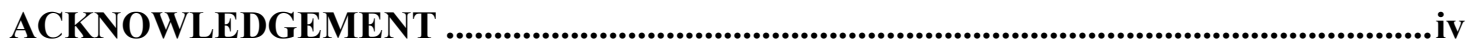

Table of Contents.........................................................................................................................................v

List of Figures ..................................................................................................................................vii

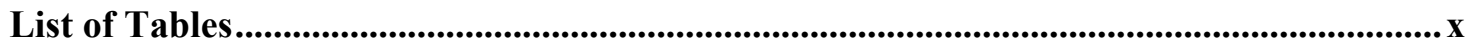

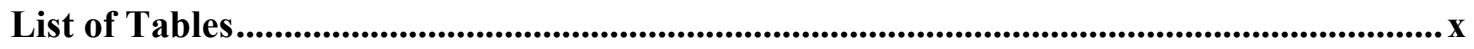

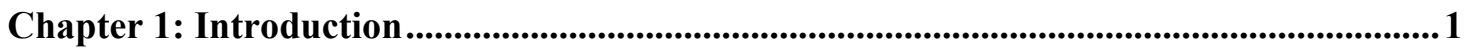

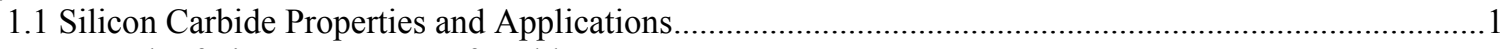

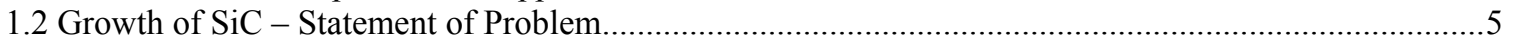

Chapter 2: Review of SiC Growth and Hydrogen-Etch Studies ............................................. 10

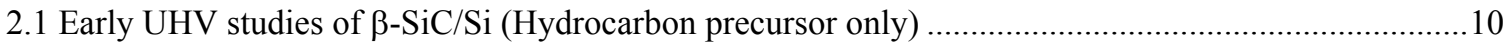

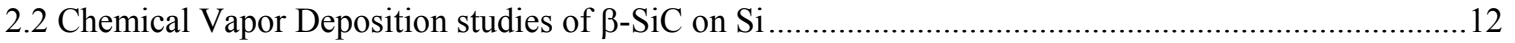

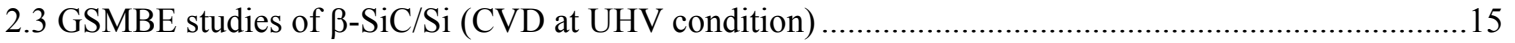

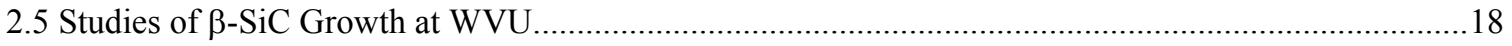

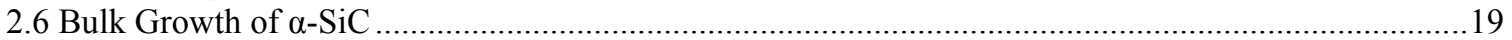

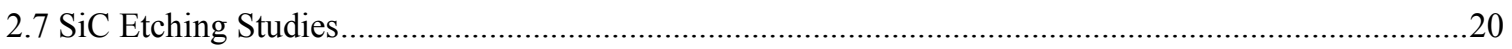

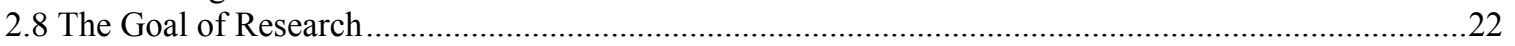

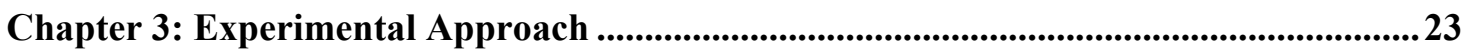

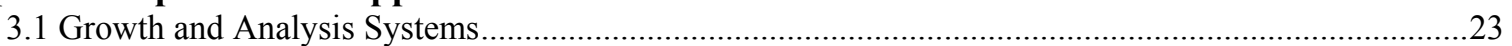

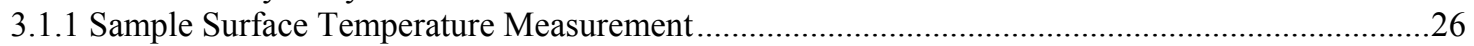

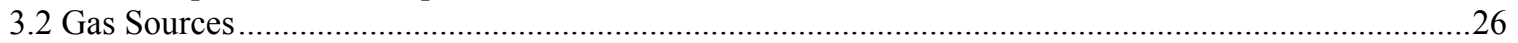

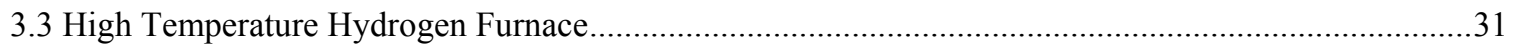

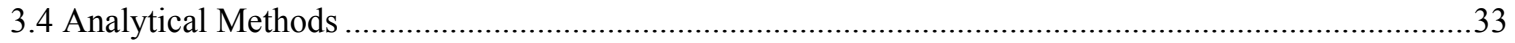

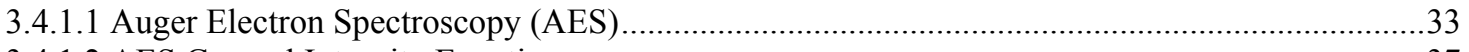

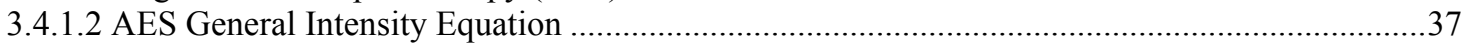

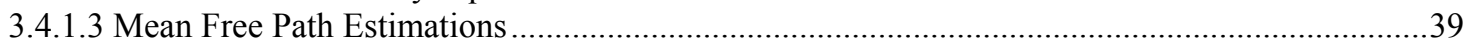

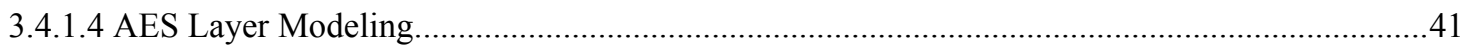

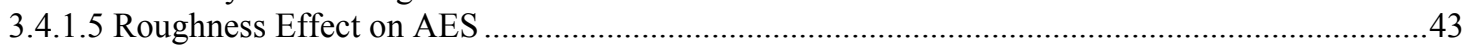

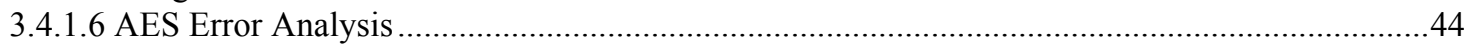

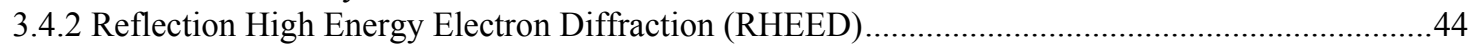

3.4.3 Field Emission Scanning Electron Microscope (FESEM) ............................................................49

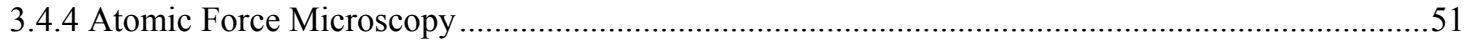

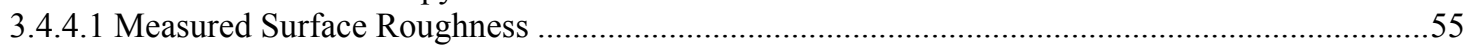

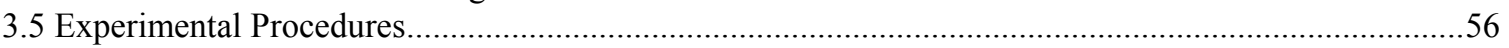

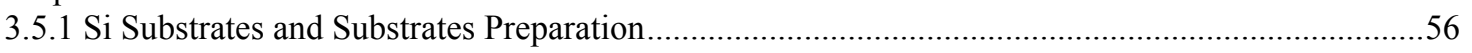

3.5.2 UHV Chamber Heteroepitaxial Growth Studies on Si Substrate ..................................................58

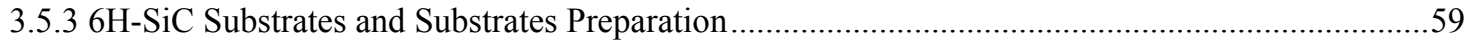

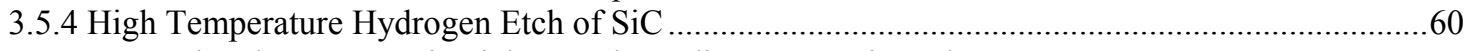

3.5.5 UHV Chamber Homoepitaxial Growth Studies on 6H-SiC Substrate ….....................................61

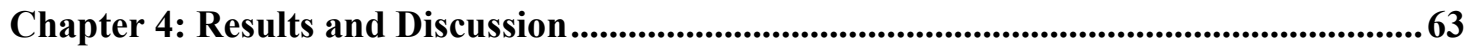

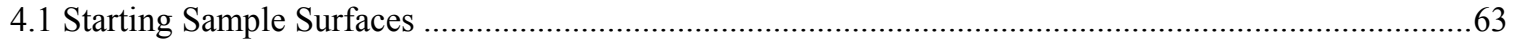

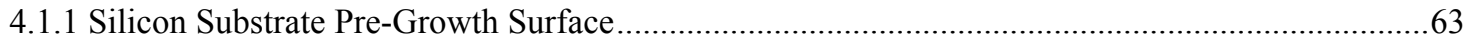

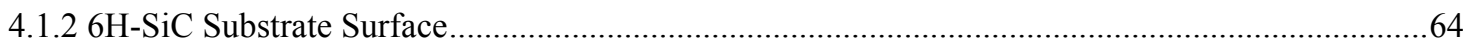

4.2 Homoepitaxial Growth on 6H-SiC Substrates …………............................................................... 
4.2.1 High Temperature Homoepitaxial Growth Using MS ..................................................................

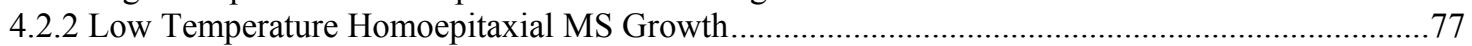

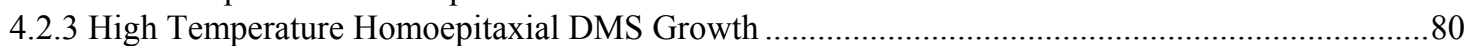

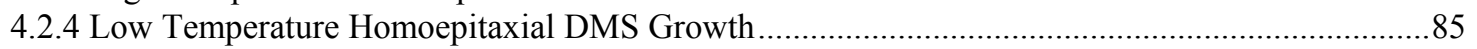

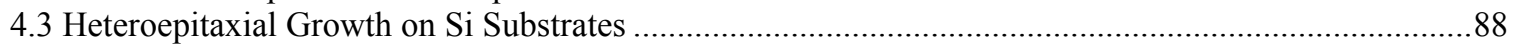

4.3.1 High Flux High Temperature Heteroepitaxial MS Growth .........................................................8

4.3.2 Low Flux High Temperature Heteroepitaxial MS Growth .........................................................92

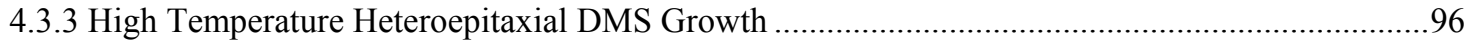

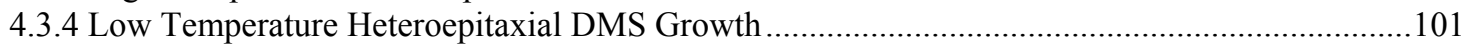

Chapter 5: CONCLUSIONS AND RECOMMENDATIONS ........................................104

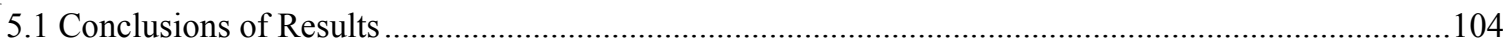

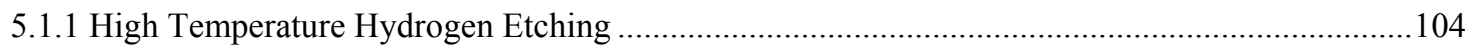

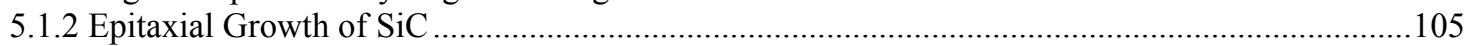

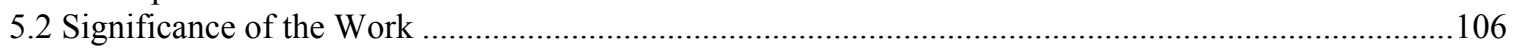

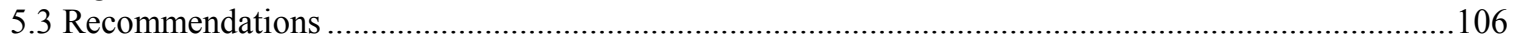

References ....................................................................................................................................................108

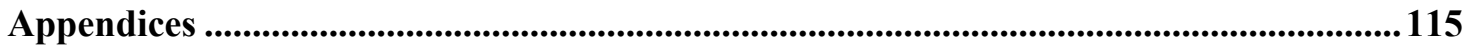

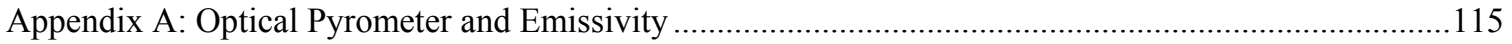

Appendix B: Relative sensitivity factor of Si-LMM and C-KLL ............................................................118 


\section{List of Figures}

Figure 3-1. Gas Source MBE system at Material and Surface Studies Laboratory at WVU....... 24 Figure 3-2. Sample holder with sample and notched end of receiver. (Courtesy: K. Ziemer) .... 25

Figure 3-3. Growth chamber gas manifold diagram............................................................. 27

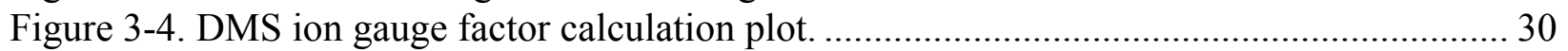

Figure 3-5. Schematics of High Temperature Hydrogen Furnace.................................................. 31

Figure 3-6. AES incident electron beam creates a hole at the core level. ...................................... 34

Figure 3-7. Auger emission occurs and the Auger electron is ejected. ....................................... 34

Figure 3-8. A differentiated AES spectrum of the substrate before and after film growth. ......... 35

Figure 3-9. Differentiated AES spectra of the $\mathrm{SiC}$, diamond, and graphite standards................. 36

Figure 3-10. Probability of emitted electrons reaching the surface............................................. 40

Figure 3-11. Auger intensity and surface roughness study. (Plot based on data extracted from

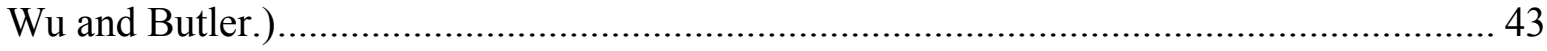

Figure 3-13. The Braggs Diffraction condition in RHEED. Concept of drawing taken from http://www.stoner.leeds.ac.uk/techniques/rheed.htm .................................................... 46

Figure 3-14. Bragg Diffraction Condition Geometry.............................................................. 48

Figure 3-15. Basic components of AFM. (Courtesy Veeco Instrument, Inc.)............................. 51

Figure 3-16. Three Common Types of AFM Tips. (a) Normal Tip ; (b) Supertip; (c) Ultralever.

Electron Micrographs by Jean-Paul Revel, Park Scientific Instruments............................. 52

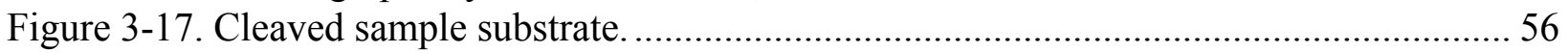

Figure 4-1. AFM images of 6H-SiC substrate as received (a), and after high temperature

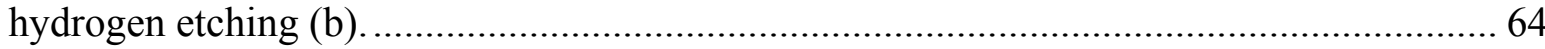

Figure 4-2. Plot of ETL versus $\mathrm{H}_{2}$ concentration for $1600^{\circ} \mathrm{C}$ and a flow rate of $8 \mathrm{SCHF}$. The

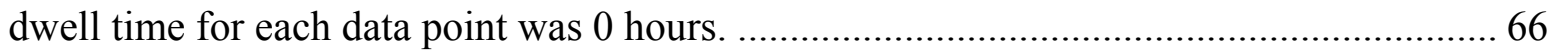

Figure 4-3. SiC etch rate versus gas composition for $1600^{\circ} \mathrm{C}$ and $8 \mathrm{SCFH}$................................ 67

Figure 4-4. 50× optical micrograph of post-etch surfaces produced by (a) $100 \% \mathrm{H}_{2}$, and (b) $5 \%$

$\mathrm{H}_{2}$ with no dwell at $1600^{\circ} \mathrm{C}$.

Figure 4-5. FESEM image of substrate etched at $1600^{\circ} \mathrm{C}$ with $5 \%$ hydrogen in $8 \mathrm{SCFH}$ argon

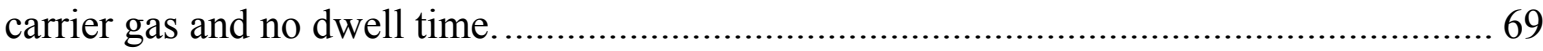

Figure 4-6. AES differentiated spectra of SiC substrate after different steps of the etching process.

Figure 4-7. Si-LMM (a) and C-KLL (b) spectra for homoepitaxial growth under HFHT conditions using MS as the gas source.

Figure 4-8. RHEED patterns for $6 \mathrm{H}-\mathrm{SiC}$ substrate before (a) and after (b) 60 minutes of growth using MS under HFHT conditions................................................................................... 73

Figure 4-9. AFM images of the $6 \mathrm{H}-\mathrm{SiC}$ substrate before growth (a) and after 60 minutes of growth using MS under HFHT conditions (b); FESEM image of the film after 15 minutes of growth using MS under HFHT conditions (c); Surface roughness and step height data before and after film growth using MS under HFHT conditions (d) .................................. 75

Figure 4-10. AFM images for 6H-SiC substrate before (a) and after (b) 15 minutes of growth using MS under HFHT conditions. 
Figure 4-11. Si-LMM (a) and C-KLL (b) spectra for homoepitaxial growth under HFLT conditions using MS as the gas source.

Figure 4-12. AFM images of the $6 \mathrm{H}-\mathrm{SiC}$ substrate before growth (a) and after 15 minutes of growth using MS under LFLT conditions (b); FESEM image of the film after 60 minutes of growth using MS under HFLT conditions (c); Surface roughness and step height data before and after film growth using MS under HFLT conditions $(\mathrm{d})$. 80

Figure 4-13. Si-LMM (a) and C-KLL (b) spectra for homoepitaxial growth under LFHT conditions using DMS as the gas source.

Figure 4-14. RHEED patterns for 6H-SiC substrate before (a) and after (b) 15 minutes of growth using DMS under HFHT conditions. 82

Figure 4-15. AFM images of the 6H-SiC substrate before growth (a) and after 15 minutes of growth using DMS under HFHT conditions (b); FESEM image of the film after 15 minutes of growth using DMS under LFHT conditions (c); Surface roughness and step height data before and after film growth using DMS under HFHT conditions (d). 83

Figure 4-16. AFM images for 6H-SiC substrate before (a) and after (b) 15 minutes of growth using DMS under HFHT conditions.

Figure 4-17. Si-LMM (a) and C-KLL (b) spectra for LFHT growth conditions using DMS as gas source. 86

Figure 4-18. AFM images of the $6 \mathrm{H}-\mathrm{SiC}$ substrate before growth (a) and after 60 minutes of growth using DMS under HFLT conditions (b); FESEM image of the film after 15 minutes of growth using DMS under HFLT conditions (c); Surface roughness and step height data before and after film growth using DMS under HFLT conditions (d).

Figure 4-19. Si-LMM (a) and C-KLL (b) spectra for heteroepitaxial growth under HFHT conditions using MS as the gas source.

Figure 4-20. RHEED patterns for Si substrate before (a) and after (b) 15 minutes of growth using MS under HFHT conditions.

Figure 4-21. AFM images of Si substrate after growth using MS under HFHT conditions for (a) 15 minutes and (b) 60 minutes; FESEM image of the film after 60 minutes of growth under HFHT conditions (c) and cross sectional view (d).

Figure 4-22. Si-LMM (a) and C-KLL (b) spectra for heteroepitaxial growth under LFHT conditions using MS as the gas source.

Figure 4-23. RHEED patterns for $6 \mathrm{H}-\mathrm{SiC}$ substrate before (a) and after (b) 15 minutes of growth using MS under LFHT conditions.

Figure 4-24. AFM images of Si substrate after growth using MS under LFHT conditions for (a) 15 minutes and (b) 60 minutes; FESEM image of the film after 60 minutes of growth under LFHT conditions (c) and cross sectional view (d).... 95

Figure 4-25. Si-LMM (a) and C-KLL (b) spectra for heteroepitaxial growth under HFHT conditions using DMS as the gas source.

Figure 4-26. FESEM images of the film after 60 minutes of growth under HFHT conditions using DMS. These images show the cross-section (a) and the surface/cross-section view of the same sample.

Figure 4-27. Differentiated Auger Si-LMM (a) and C-KLL (b) peak line shapes comparison at $800^{\circ} \mathrm{C}$ for low flux runs.

Figure 4-28. FESEM images of the film after 60 minutes of growth under LFHT conditions using DMS. There images show the cross-section (a) and the surface (b) view of the same sample. 100 
Figure 4-29. RHEED patterns for the Si substrate before (a) and after (b) 15 minutes of growth with DMS under LFHT conditions..................................................................... 100

Figure 4-30. Si-LMM (a) and C-KLL (b) spectra for heteroepitaxial growth under LFLT conditions using DMS as the gas source.

Figure 4-31. Si-LMM (a) and C-KLL (b) spectra for heteroepitaxial growth under HFLT conditions using DMS.

Figure A-1. Black body Optical Pyrometer calibration including losses caused by windows... 115

Figure A-2. Emissivity of $6 \mathrm{H}-\mathrm{SiC}$ at substrate temperature range of $600^{\circ} \mathrm{C}-800^{\circ} \mathrm{C} \ldots \ldots \ldots \ldots \ldots \ldots . . .117$

Figure A-3. AES survey spectrum for 60 minutes of heteroepitaxial growth under HFHT conditions using MS. 


\section{List of Tables}

Table 1-1. Properties of Important Semiconductors ………......................................................... 2

Table 4-1. Summary of selected hydrogen-etch runs and results .................................................. 68 


\section{Chapter 1: Introduction}

\subsection{Silicon Carbide Properties and Applications}

Silicon carbide $(\mathrm{SiC})$ is an advanced semiconductor suitable for electronic devices and sensors that can operate in hostile environments where conventional silicon based electronics cannot function properly or effectively. In recent years, the activity in $\mathrm{SiC}$ research has considerably increased due to the need for electronic devices capable of functioning in high temperature, high power, and high radiation conditions. The broad applications areas included in this are aircraft, aerospace, automotive, transportation, nuclear and fossil fuel generation. ${ }^{1} \mathrm{SiC}$ 's ability to function under such extreme conditions may be traced to its physical properties. In particular, the large Si-C bond energy makes it stable in high temperature, its high electron mobility, high electron saturation velocity, and high thermal conductivity make it a promising semiconductor material for such purposes. ${ }^{2}$

$\mathrm{SiC}$ exists in approximately 250 different atomic polytypes, i.e. different crystal structures. ${ }^{3}$ These polytypes are obtained by different structural arrangements of Si-C bi-layers and fall into two broad structure types, hexagonal and cubic. ${ }^{4}$ Each polytype can be described by the number of layers per period along the stacking direction followed by the first letter of the crystal system (C-cubic; H-hexagonal). These two structure systems may also be referred to as $\beta$-SiC and $\alpha-\mathrm{SiC}$ respectively. Commonly occurring examples of $\alpha$-SiC include $2 \mathrm{H}-, 4 \mathrm{H}-$, and $6 \mathrm{H}-\mathrm{SiC} . \quad \beta-\mathrm{SiC}$ or $3 \mathrm{C}-\mathrm{SiC}$ is the only cubic polytype and has a zinc blende cubic structure. The physical properties of the most common $\mathrm{SiC}$ polytypes are compared to the properties of $\mathrm{Si}$ in Table $1-1.5$ 
Table 1-1. Properties of Important Semiconductors

\begin{tabular}{|c|c|c|c|c|}
\hline & $\mathrm{Si}$ & $6 \mathrm{H}-\mathrm{SiC}$ & $4 \mathrm{H}-\mathrm{SiC}$ & $3 \mathrm{C}-\mathrm{SiC}$ \\
\hline Max. Operating Temp. $\left({ }^{\circ} \mathrm{C}\right)$ & 250 & 300 & 1100 & 750 \\
\hline Band Gap (eV) & 1.11 & 3.02 & 3.26 & 2.23 \\
\hline Thermal Expansion Coeff. $\left(10^{-6}{ }^{\circ} \mathrm{C}^{-1}\right)$ & 2.33 & 2.6 & 4.2 & 2.5 \\
\hline Young's Modulus (GPa) & 47 & 190 & 1035 & 448 \\
\hline Lattice constant $(\AA)$ & 5.43 & 3.09 & 3.09 & 4.36 \\
\hline Breakdown Field@1kV Operation(MV/cm) & 0.3 & $3 \sim 5$ & $3 \sim 5$ & $>1$ \\
\hline Electron Mobility@10 $10^{16} \mathrm{~cm}^{-3}\left(\mathrm{~cm}^{2} / \mathrm{V}-\mathrm{s}\right)$ & 1100 & 370 & 800 & 750 \\
\hline Thermal Conductivity (W/cm-K) & 1.5 & 4.9 & 4.9 & 5.0 \\
\hline $\begin{array}{l}\text { Saturated Electron Drift Velocity }\left[10^{6} \mathrm{~cm} / \mathrm{sec}\right. \\
\left(\left(@ 2 \times 10^{5} \mathrm{~V} / \mathrm{cm}\right)\right]\end{array}$ & 420 & 90 & 115 & 40 \\
\hline
\end{tabular}

Bandgap is defined in short as the forbidden range of energies between the valence and the conduction bands in an insulator or semiconductor. Before an electron in the valance band can be excited into the conduction band (where it can contribute to electrical conduction), it must be stimulated with an energy that is equal to or greater than the bandgap energy. Insulators have a large bandgap, typically greater than $5 \mathrm{eV}$, making it difficult to excite electrons into the conduction band. In comparison, a semiconductor, such as $\mathrm{Si}$, has a relatively narrow bandgap $(1.1 \mathrm{eV})$. A wide bandgap semiconductor is generally defined as one having a band gap greater than that of Si.

At room temperature even the $1.1 \mathrm{eV}$ band gap of $\mathrm{Si}$ is sufficiently wide to prevent any significant thermal excitation of electrons across the band gap. Consequently, electrical conductivity can be controlled through intentional doping of the semiconductor using donor and 
acceptor atoms. As the temperature increases above $300^{\circ} \mathrm{C}$, significant thermal excitation may occur. This is a major reason why Si-based devices fail at temperature above $250^{\circ} \mathrm{C}$. The larger bandgap provides a greater energy barrier against thermal excitation and allows $\mathrm{SiC}$ device operation at temperatures close to $750^{\circ} \mathrm{C} .^{6}$

$\mathrm{SiC}$ can also support very high breakdown fields of $3.0 \mathrm{MV} / \mathrm{cm}$ for $4 \mathrm{H}$ and $6 \mathrm{H}$ polytypes, five times higher than that of $\mathrm{Si}(0.6 \mathrm{MV} / \mathrm{cm})$ at comparable doping levels. As a consequence, SiC-based devices allow very high voltage and high power operations.

Another critical parameter for power producing devices is thermal conductivity. The outstanding thermal conductivity of quality $\mathrm{SiC}$-approximately three times better than $\mathrm{Si}-$ allows SiC-based devices to more efficiently remove waste heat, and to operate at a lower junction temperature, which improves device lifetime and reliability.

Although the electron mobility of $\mathrm{SiC}$ is not as good as $\mathrm{Si}$, it is sufficient in the high electric field regimes where $\mathrm{SiC}$ devices typically operate. However, for ever increasing fields, drift velocity becomes saturated and no longer increases with increasing electrical field. Of course, the maximum speed of devices operated at high field strengths is directly related to this quantity. That is where $\mathrm{SiC}$ has the advantage; simply comparing mobilities could put $\mathrm{SiC}$ at a disadvantage.

These $\mathrm{SiC}$ properties enable a large range of applications from greatly improved highvoltage switching ${ }^{7}$ for energy savings in public electric power distribution and electric vehicles to more powerful microwave electronics for radar and communications ${ }^{8}$ to sensors and controls for cleaner-burning, more fuel-efficient combustion chambers for jet aircraft, automobile engines, and for stationary power plants. ${ }^{9,} 10$ Theoretical appraisals indicate that $\mathrm{SiC}$ devices will operate over higher voltage and temperature ranges, have superior characteristics, and yet have a 
die size (surface area size) nearly 20 times smaller than correspondingly rated Si-based devices. $^{11}$

Silicon carbide has also found an application as a substrate material for gallium nitride $(\mathrm{GaN})$, because of the relatively close lattice match with III-nitrides and its high thermal conductivity. GaN attracts much interest due to its potential applications in the fields of optoelectronics and electronics. Because of the large and direct gap as well as its outstanding chemical and physical stability, this material is a great candidate for the construction of shortwavelength light emitting devices and transistors operating at high temperature. ${ }^{12,13}$ The use of GaN has made possible the commercial viability of blue and green output laser diodes. The current generation of data storage devices such as DVD players uses a 650nm red output laser diode. A reduction in the wavelength to $400 \mathrm{~nm}$, possible using GaN-based laser diodes, will allow a five-fold increase in storage capacity. Crystal growth for GaN lasers has been performed using sapphire substrates. This usually results in a high dislocation density that shortens laser life.

Another advantage of $\mathrm{SiC}$ as a $\mathrm{GaN}$ substrate is that $\mathrm{SiC}$ is electrically conductive whereas sapphire, the common growth substrate for $\mathrm{GaN}$, is an insulator. This allows electrodes to be formed on both sides thus reducing the size and the cost of extra electrodes. The optoelectronic properties of $\mathrm{GaN}$ have the potential to make the incorporation of $\mathrm{SiC}$ materials a key technology in the fabrication of optoelectronic integrated circuits. Specifically, the luminescence in the blue and ultraviolet regions of GaN has attracted much interest.

These predicted (and, in some cases, demonstrated on a laboratory scale) advantages have yet to be realized in commercial applications. This is due mainly to limited reproducibility of SiC's relatively immature growth technologies. 


\subsection{Growth of SiC - Statement of Problem}

The major goal of the $\mathrm{SiC}$ growth community for the past few decades has been the production of large area, high quality $\mathrm{SiC}$ substrates. Today, bulk growth of large area $\alpha$-SiC substrates are typically grown using high temperature physical vapor transport (PVT) methods. ${ }^{14}$ PVT growth involves the sublimation of a polycrystalline $\mathrm{SiC}$ source $(\mathrm{SiC}$ does not melt, it instead sublimes at $1825^{\circ} \mathrm{C}$ ) placed in the hot zone of a graphite crucible acting as the growth furnace and the subsequent mass transport of the vapor species to the seed crystal located in a cooler region of the furnace, usually at a temperature a few hundred of degrees lower than the source. A temperature gradient of $20-40^{\circ} \mathrm{C} / \mathrm{cm}$ is maintained over the length of furnace. Single crystal $\mathrm{SiC}$ is grown by deposition of the supersaturated vapor species $\left(\mathrm{Si}, \mathrm{SiC}_{2}, \mathrm{Si}_{2} \mathrm{C}\right.$, etc.) onto the seed crystal, creating a crystal of a single polytype. The origin of this was the Acheson Process ${ }^{15}$ and later refined by Lely in $1955^{16}$, and as now practiced, is capable of producing single crystal ingots up to 4 inches in diameter. Typical $6 \mathrm{H}-$ and $4 \mathrm{H}-\mathrm{SiC}$ bulk source temperature is $2100-2400^{\circ} \mathrm{C}$, growth pressure is less than 20 Torr, and growth rates for the bulk growth of $\mathrm{SiC}$ are in the range of $0.5-5 \mathrm{~mm} / \mathrm{hr} .{ }^{17,18}$ Lower vapor pressure or higher growth temperature (gradient) increases the growth rate. During growth, gaseous $\left(\mathrm{N}_{2}\right)$ as well as solid $(\mathrm{B}, \mathrm{Al}, \mathrm{V})$ dopants can be used to adjust the electrical properties of the growing semiconductor crystal. Due to the extreme hardness and atomic density of the material, doping after growth by diffusion or ion implantation only leads to very thin doped layers. Such sublimation techniques are relatively easy to implement, but these processes are difficult to control, particularly over large substrate areas at the high growth temperatures required.

The major factor limiting the use of $\alpha-\mathrm{SiC}$ substrates is the high density of defects known as micropipes. A micropipe is a tube-like defect (with diameter ranging from fractions of a 
micron to tens of microns) that extends through the $\mathrm{SiC}$ crystal. ${ }^{19}$ The origin of micropipes is still not completely understood, but it is widely believed that micropipes are several screw dislocations clustered together so severely that an empty core is formed in the center. Frank showed that a dislocation whose Burgers vector exceeds a critical value, of the order of magnitude $10 \AA$, is only in equilibrium with an empty tube at its core. ${ }^{20}$ Burger's vector denotes magnitude and direction of lattice distortion. The density of micropipe defects in commercial $\mathrm{SiC}$ wafers may exceed $100 \mathrm{~cm}^{-2}$ during device fabrication. In subsequent epitaxial growth, micropipes originating from the $\mathrm{SiC}$ substrate can propagate into device structures to cause device failure. Understandably, the presence of these micropipes unkindly compromises the device performance of the $\mathrm{SiC}$ substrates. ${ }^{21}$ Further typical defects in $\mathrm{SiC}$ crystals are planar voids, silicon and carbon inclusions, stacking faults and polytype changes. ${ }^{22}$ In addition, the substrates are generally composed of domains with slightly different orientation which thereby will give a mosaic structure. ${ }^{23}$ These domains are revealed after etching in molten potassium hydroxide $(\mathrm{KOH})$ and large micropipes are often seen at the boundaries between different domains. $^{24}$

Wafer cost is another factor limiting the use of $\alpha$-SiC wafers. Price ranges from $\$ 1,850$ to $\$ 9,000$ per 3-inch (diameter) wafer depending on the grade and micropipe density compared to the $\$ 4.75$ cost of a 4 -inch high quality $\mathrm{Si}$ wafer. ${ }^{25}$ Combining this with their undesirable quality, $\alpha$-SiC wafers presently have very limited industrial application.

Commercially available $\alpha-\mathrm{SiC}$ wafers are sliced and polished (one side) off-axis usually at an angle between $3^{\circ}$ and $8^{\circ}$ (tilts of less than $1^{\circ}$ are considered on-axis). The use of off-axis substrates exposes steps on the growth surface of several alternating silicon and carbon planes, thereby transferring stacking information onto the subsequently epitaxially grown film. 
However, the polishing process introduces new defects in the form of micro-scratches invisible to bare eyes but detrimental to device quality.

Current research in bulk crystal growth centers on increasing the growth rate, reducing material defects, and increasing the substrate diameter to at least 4 inches (so the current silicon wafer processing equipment could still be used).

As an alternative to the use of $\alpha$-SiC wafers, many groups have attempted to use $\beta$-SiC films grown on large area $\mathrm{Si}$ wafers. $\beta$-SiC films are traditionally produced using atmospheric pressure chemical vapor deposition (APCVD) and low pressure gas source molecular beam epitaxy (GSMBE). Although GSMBE has the advantage of potentially lower growth temperature, both suffer from the fact that $\mathrm{Si}$ and $\mathrm{SiC}$ are not closely matched in key physical properties. In most cases, growth is performed on single-crystal Si (100) surfaces for which the lattice mismatch is $\sim 20 \%$ ( $\mathrm{Si}$ has a lattice constant of $5.43 \AA$ while $\mathrm{SiC}$ is only $4.36 \AA$ ). High growth temperature of up to $1200^{\circ} \mathrm{C}-1400^{\circ} \mathrm{C}$ are required. This also poses a problem due to the thermal expansion mismatch between $\mathrm{SiC}\left(2.5 \times 10^{-6} / \mathrm{K}\right)$ and $\mathrm{Si}\left(2.3 \times 10^{-6} / \mathrm{K}\right)$, whereas the film and substrate undergo dimensional changes upon cooling after growth. Mismatch in thermal expansion creates a significant amount of stress in the film. In addition, high growth temperature can also result in formation of voids at the $\mathrm{SiC} / \mathrm{Si}$ interface, in the Si substrate, and of dislocations in the $\mathrm{SiC}$ film as well. ${ }^{26}$ Lower temperature epitaxy is thus desirable for device fabrication with heteroepitaxially grown $\beta-\mathrm{SiC}$ on $\mathrm{Si}$. There is an additional problem in that $\mathrm{Si}$ being highly reactive and mobile, and it often diffuses through the film to the reaction surface to form $\mathrm{SiC}$. As a result, pits and voids are often formed at the $\mathrm{Si}-\mathrm{SiC}$ interface and serve as the source of surface irregularities in the film. Without significant improvement, it is unrealistic to 
expect that $\beta$-SiC films produced by today's method can be used for electronic devices even though some MEMS devices have been made from pseudo-wafers of polycrystalline $\beta$-SiC. ${ }^{27}$

As means of overcoming these problems associated with $\beta$-SiC growth, the generally preferred method is to form a very thin $\mathrm{SiC}$ "carbonized layer" by reacting a carbon-containing precursor with the Si surface before actual growth of $\mathrm{SiC}$ begins. This layer is also referred to as a "conversion layer" or "buffer layer", and its primary purposes are to reduce the lattice mismatch and Si out-diffusion.

Once the conversion layer is formed, $\mathrm{SiC}$ film growth usually proceeds using a combination of Si- and C- containing gases. Conventionally, the $\mathrm{Si}$ is provided by using $\mathrm{SiH}_{4}$ as a source gas. From a safety stand point, this poses a problem in that $\mathrm{SiH}_{4}$ is pyrophoric (autoigniting in air at concentration of $>10 \%$ ) and extremely dangerous. $\mathrm{SiCl}_{4}$ has been used as the $\mathrm{Si}$ precursor, but it is corrosive to process equipment.

As an alternative to the use of $\mathrm{SiH}_{4}$, the methylsilane family of source gases (methylsilane, dimethylsilane, trimethylsilane, and tetramethylsilane) present some interesting possibilities. They are less corrosive and inherently more stable than silane. Methylsilane is reported to be pyrophoric in limited cases but still more stable than $\mathrm{SiH}_{4}$. In addition, the family of precursors serves as a single source of both Si and carbon.

Recent studies of GSMBE growth of $\mathrm{SiC}$ using methylsilane $\left(\mathrm{H}_{3} \mathrm{SiCH}_{3}\right)$ reported successful growth of $\beta$-SiC film on $\mathrm{Si}(100)$ at $900^{\circ} \mathrm{C} .{ }^{28}$ However, there is very limited information on growth characteristics in regard to the effect of growth conditions (temperature, pressure, time, and substrate pretreatment). Moreover, there is even less information concerning the use of other members of the methylsilane family. 
The goals of the present research are to develop GSMBE methods of growing smooth, epitaxial $\mathrm{SiC}$ films on $\mathrm{Si}$ and $\alpha$-SiC using novel precursors and methods of controlling the substrate surface structure. When combined with previous work from this laboratory on trimethylsilane and work performed elsewhere on methylsilane, the proposed studies will provide a more comprehensive picture of GSMBE growth of SiC growth using this family of precursors.

The specific objectives of the study are:

1) To use a high temperature furnace to hydrogen-etch $\alpha$-SiC substrates and create an ordered surface for subsequent epitaxial growth. This step is parallel to the Fenner Etch method for Si substrate, which is a hydrofluoric acid-based wet chemical etch method that produces a flat hydrogen-terminated $\mathrm{Si}(1 \times 1)$ surface.

2) To grow smooth, epitaxial, and high quality films on both $\alpha$-SiC and Si substrates using methylsilanes as gas sources, upon which further epitaxial growth and device fabrication are suitable.

3) To examine the kinetics of the initial reactions of methylsilanes on Si substrates. 


\section{Chapter 2: Review of SiC Growth and}

\section{Hydrogen-Etch Studies}

\subsection{Early UHV studies of $\beta$-SiC/Si (Hydrocarbon precursor only)}

Studies of SiC films formed by the reaction of single-crystal Si with gaseous hydrocarbon precursors at elevated temperatures can be generally categorized into two groups: reaction in high and ultra high vacuum chambers with the source gases introduced through a controlled leak valve or molecular beam; or reaction in a flowing gas mixture (source mixed in carrier gas) at or near atmospheric pressure. The former will be referred to as GSMBE, while the latter will be referred to as atmospheric pressure chemical vapor deposition (APCVD) or reduced pressure chemical vapor deposition (RPCVD).

GSMBE of SiC using single and multiple precursors has been studied extensively. In 1967, Khan and Summergrad first reported the successful growth of single-crystal films of $\beta$-SiC under ultrahigh vacuum (UHV) conditions. ${ }^{29}$ The single-crystal films were grown on $\mathrm{Si}$ substrates within the temperature range $800-1000^{\circ} \mathrm{C}$ using $\mathrm{C}_{2} \mathrm{H}_{2}$ and $\mathrm{C}_{2} \mathrm{H}_{4}$ with Si. Khan and Summergrad reported films up to $1000 \AA$ thick that were single crystalline and showed no polycrystalline character. The $\mathrm{SiC}$ grew with a parallel epitaxial relationship to the $\mathrm{Si}$ substrate, regardless of the substrate orientation used. At the onset of the experiment, the Si substrates

were mounted on a tantalum heater in a UHV system at a base pressure of $2 \times 10^{-9}$ Torr and hydrocarbon precursor was released into the system through a leak valve to control its partial 
pressure. The growth temperature ranged from 800 to $1000^{\circ} \mathrm{C}$, and films were much thicker when formed at higher temperatures indicating higher growth rate. The authors reported that films of several microns thick were produced at a $\mathrm{C}_{2} \mathrm{H}_{2}$ partial pressure of $2 \times 10^{-5}$ Torr, compared to up to $1 \mathrm{~nm}$ at $3 \times 10^{-7}$ Torr under otherwise similar conditions. This study also revealed the important role vacuum conditions play in the growth of $\mathrm{SiC}$ films. Films produced by maintaining the substrate under UHV prior to the hydrocarbon reactions showed greater perfection than those maintained under normal HV conditions. In the latter case, the SiC films showed some polycrystalline character and defects such as stacking faults and dislocations. According Khan and Summergrad, ${ }^{22}$ UHV conditions "appear necessary for growing singlecrystal films of high quality."

Following the study of Khan and Summergrad, Mogab and Leamy continued on the same path in an attempt to better understand $\beta$-SiC growth, particularly the vacuum-growth process, the effect of the vacuum environment (residual gas contaminants), and the origin of the characteristic defects. ${ }^{30}$ In these studies, the growth of $\beta$-SiC films on Si was achieved by the reaction of a Si single-crystal surface with $\mathrm{C}_{2} \mathrm{H}_{2}$ at partial pressure between $10^{-7}$ and $5 \times 10^{-4}$ Torr and temperature between 800 and $1100^{\circ} \mathrm{C}$, in both $\mathrm{HV}$ and UHV chambers. No significant difference in growth rates was observed between HV and UHV under otherwise identical conditions, although the residual vacuum conditions certainly played an important role in the film quality. In several experiments conducted in which Si wafers were intentionally exposed to pump oils but no $\mathrm{C}_{2} \mathrm{H}_{2}$ during the reaction, $\mathrm{SiC}$ growth occurred. Initial surface preparation methods also had no substantial effect on growth rate although layers grown in UHV showed a higher degree of crystalline perfection if the Si crystal was annealed prior to reaction. This helped obtain a clean Si surface. In studying the growth conditions, it was found that films 
grown at higher pressure ( $\geq 10^{-5}$ Torr) and temperatures were often thinner than films grown for equivalent times at $\mathrm{C}_{2} \mathrm{H}_{2}$ pressures a factor of 10 less. With the fact that $\mathrm{SiC}$ layer thickness under such conditions showed vast randomness, the authors concluded after some relatively short initial period there is little or no further growth. That is, the growth process stops. There were insufficient data to determine the temperature dependence of the growth rate constant other than the fact that increasing temperature increases the rate.

Optical microscopy of films formed at all but the highest pressures $\left(>10^{-5}\right.$ Torr) revealed "growth defects" similar to those observed in some earlier work. It was explained that low initial growth rate due to lower $\mathrm{C}_{2} \mathrm{H}_{2}$ pressure resulted in defects in the form of shallow pits in the Si crystal, and the $\mathrm{SiC}$ layer that over-grows these pits assumes "porous" polycrystalline morphology. Considering the impermeability of the defect-free porous region of the film, the $\mathrm{Si}$ was supplied to the reaction surface primarily by means of surface diffusion through these porous regions and subsequent lateral surface diffusion over the defect-free film surface. Higher $\mathrm{C}_{2} \mathrm{H}_{2}$ pressures ( $>10^{-5}$ Torr) resulted in higher initial growth rates and a sealing off of the defect channels at an early stage in the growth process and thus, produced a more defect free surface. Finally, it was determined that defect growth is concurrent with $\mathrm{SiC}$ film growth and the density of such defects increases linearly with film thickness, regardless of reaction conditions $\left(5 \times 10^{-7}<\right.$ $\mathrm{P}_{\text {acetylene }}<1 \times 10^{-5}$ Torr; $\left.800<\mathrm{T}<1050^{\circ} \mathrm{C}\right)$.

\subsection{Chemical Vapor Deposition studies of $\beta-\mathrm{SiC}$ on $\mathrm{Si}$}

Chemical Vapor Deposition (CVD) involves chemical reactions that transform gaseous molecules, called precursors, into a solid material in the form of a thin film or powder on the surface of a substrate. The process, widely used to fabricate semiconductor devices, consists of six steps: (1) transport or bulk gas flow of precursor molecules into and through the high 
temperature region above the substrate; (2) gas phase decomposition and reaction of these reactants to form a gas phase mixture of precursor and intermediate reaction product called "growth species" as they diffuse to the surface; (3) adsorption of the growth species (with and without decompositions) onto the surface; (4) the diffusion of the adsorbed species to active surface sites where they react to form the thin film: (5) desorption of reaction product molecules from the substrate or thin film surface; (6) diffusion of products away from the substrate and into the bulk gas flow. In a typical CVD process, reactant gases molecules (diluted in a carrier gas) enter the reaction chamber at room temperature. The gas mixture is heated by convection/radiation as it flows over the heated substrate. Depending on the process and operating conditions, the homogeneous chemical reactions to form the growth species may be under equilibrium or kinetic control. Heterogeneous reactions of the growth species to form the thin film may also be kinetically controlled. The desorption of reaction by-products from the surface to open a potential reaction site for incoming growth species may also be a rate limiting step.

In the early 1980's, Nishino et al. reported the first truly successful approach for atmospheric pressure chemical vapor deposition (APCVD) $\beta$-SiC growth. ${ }^{31} \mathrm{~A}$ buffer or "conversion" layer, formed by reaction of the Si substrate surface with a carbon-containing gaseous species, was used between the cubic SiC and the Si substrate to minimize the effect of lattice mismatch. ${ }^{26}$

A thin film of $\beta$-SiC was deposited onto $\mathrm{Si}$ by $\mathrm{RF}$ sputtering at $800-1000^{\circ} \mathrm{C}$. The resulting conversion or buffer layer was on the order of 50-100 nm thick. The subsequent deposition of $\beta$-SiC was carried out at a substrate temperature in the range of $1000-1360^{\circ} \mathrm{C}$ with 
0.080 mole $\% \mathrm{SiH}_{4}$ and 0.026 mole \% propane in hydrogen. Under these conditions, different thicknesses of $\mathrm{SiC}$ single-crystal layer could be grown on the buffer layer by regulating growth time. The surface was covered with rounded grains, the size of which became larger with increasing substrate temperature. When the substrate temperature was above $1360^{\circ} \mathrm{C}$, square crystallites were observed on the layer grown on the (100) Si substrate and triangular crystallites on a (111) substrate. When the sputtered layer was grown at temperature lower than $800^{\circ} \mathrm{C}$, the subsequent layer grown by CVD was not a single crystal even if a high substrate temperature was employed during the CVD process. Overall, single crystal of $\beta$-SiC was obtained reproducibly by $\mathrm{CVD}$ at $1360^{\circ} \mathrm{C}$ on a $\mathrm{Si}$ substrate when using sputtered $\mathrm{SiC}$ layers as buffer layers. The researchers speculated that the growth of the single crystalline $\beta$-SiC was controlled by the "crystal field of the Si substrate through the polycrystalline intermediate buffer layer formed by sputtering". Single-crystal $\beta$-SiC growth up to $4 \mu \mathrm{m}$ was achieved during this study and the same approach was extended further by growing the buffer layer in-situ in the CVD system and thicknesses up to $20 \mu \mathrm{m}$ were reached. ${ }^{32}$

Nishino later joined Powell and Will at NASA's Lewis (Glenn) Research Center in an attempt to improve this process. Specifically, their goal was to reproducibly fabricate singlecrystal layers that were thicker, large in area, and of higher quality. ${ }^{26}$ This study adopted most of the methods and techniques employed in Nishino's previous work but paid special attention to the cleanness of the CVD system and the precise gaseous flow and temperature control. A procedure was used whereby the complete gas handling system is continually purged with highpurity Ar between growth runs. A Si/C ratio of less than $1\left(\mathrm{SiH}_{4} / \mathrm{C}_{3} \mathrm{H}_{8}\right.$ mole ratio less than 3$)$ was used. This buffer layer method for growing a SiC layer on a Si substrate consists of three distinct steps. During each of the steps the purified hydrogen gas flow is maintained while the 
desired process gases are added. Once the Si substrate is etched with $\mathrm{HCl}$ gas at $1200^{\circ} \mathrm{C}$, it was heated to $1400^{\circ} \mathrm{C}$ in the presence of 0.03 mole $\% \mathrm{C}_{3} \mathrm{H}_{8}$ for about a minute, concluding the growth of the buffer layer. The Si wafer was then cooled and reheated to $1400^{\circ} \mathrm{C}$ with 0.04 mole $\% \mathrm{SiH}_{4}$ and 0.02 mole \% propane. Under these conditions, different thicknesses of SiC singlecrystal layer can be grown on the buffer layer by regulating growth time. (Typical growth rates were about $2.5 \mu \mathrm{m} / \mathrm{h}$.) The buffer layer grown was determined to be about $20 \mathrm{~nm}$ thick and probably mostly polycrystalline cubic $\mathrm{SiC}$, but the exact nature and role of this layer were still not fully understood at that time. Overall, the process consistently yielded higher-quality singlecrystal cubic SiC layers that are uniform over the whole substrate. X-ray diffraction measurements and Laue photographs have verified that the crystals are cubic SiC single-crystals and showed no evidence of other SiC polytypes present, indicating very good crystal quality.

\subsection{GSMBE studies of $\beta-\mathrm{SiC} / \mathrm{Si}$ (CVD at UHV condition)}

Growth of $\beta$-SiC on Si in UHV using a combination of Si- and C- containing precursors was tried in the $1980 \mathrm{~s}$. Low-temperature growth of $\beta$-SiC heteroepilayers was reported using several combinations of precursors, e.g., $\mathrm{SiH}_{4} / \mathrm{CH}_{4},{ }^{33} \mathrm{SiHCl}_{3} / \mathrm{C}_{2} \mathrm{H}_{4},{ }^{34}$ and $\mathrm{Si}_{2} \mathrm{H}_{6} / \mathrm{C}_{2} \mathrm{H}_{2}{ }^{35}$ Similar to chemical vapor deposition, a buffer layer was grown on the Si substrate and carbonization by exposure to $\mathrm{C}$-containing precursors was carried out prior to the $\beta$-SiC growth in each case to improve the crystal quality of the heteroepilayers. In the heteroepitaxy of $\beta$-SiC on $\mathrm{Si}$, a gradual temperature rise during carbonization together with the existence of a surface oxide layer was effective to moderate the reaction between $\mathrm{Si}$ and $\mathrm{C}_{2} \mathrm{H}_{2}$ and a single crystalline $\beta$-SiC thin layer could be obtained reproducibly at $970^{\circ} \mathrm{C} .^{36}$ By the use of chemically active hydrocarbon radicals from thermal cracking of $\mathrm{C}_{3} \mathrm{H}_{8}$, a Si surface could be carbonized reproducibly at temperatures as low as $750^{\circ} \mathrm{C}^{37}$ A single-crystalline $\beta$-SiC layer showing a $(3 \times 2)$ structure was obtained at 
$1000^{\circ} \mathrm{C}^{38}$ Matsunami et al. investigated one such case focusing on different carbonization processes including $\mathrm{C}_{2} \mathrm{H}_{2}$, thermal cracking of $\mathrm{C}_{3} \mathrm{H}_{8}$, and dimethylgermane (DMGe) $\left(\mathrm{CH}_{3}\right)_{2} \mathrm{GeH}_{2}$ to chemically convert the surface region into single crystalline $3 \mathrm{C}-\mathrm{SiC}$ prior to crystal growth. ${ }^{39}$ In the case of using acetylene $\left(\mathrm{C}_{2} \mathrm{H}_{2}\right)$, a carbonized layer with a smooth surface was achieved by a modified carbonization process at $970^{\circ} \mathrm{C}$. When the $\mathrm{C}_{2} \mathrm{H}_{2}$ flow rate is small, the $\beta$-SiC layer is not thick enough to prevent out-diffusion of Si atoms from the substrate. With the increase of $\mathrm{C}_{2} \mathrm{H}_{2}$ flow rate, the defects are sealed off at an early stage and the Si surface is covered with a single-crystalline $3 \mathrm{C}-\mathrm{SiC}$ layer that prevents out-diffusion of $\mathrm{Si}$ atoms and thus a degradation of the crystallinity did not occur. However, optimum growth conditions such as flow-rate of $\mathrm{C}_{2} \mathrm{H}_{2}$ and the rate of substrate temperature increase were very narrow, and the carbonized layer easily became polycrystalline. Using methyl radicals $\left(\mathrm{CH}_{3}\right)$ from thermal cracking of propane $\mathrm{C}_{3} \mathrm{H}_{8}$, single crystalline 3C-SiC with a mirror-like surface could be obtained reproducibly at a temperature as low as $750^{\circ} \mathrm{C}$. The success of carbonization at such a low temperature is believed to be due to reactions of chemically active $\mathrm{CH}_{3}$ radicals with $\mathrm{Si}$ atoms on the surface. When pure DMGe was used as a source gas, a clean Si surface could be carbonized reproducibly even at a temperature as low as $650^{\circ} \mathrm{C}$. The carbonized layer had good crystallinity without any $\beta$ $\mathrm{SiC}$ twin spots and Ge-related diffraction patterns. The success of carbonization at such a low temperature in this experiment was because most of the Ge atoms were desorbed while the active $\mathrm{CH}_{3}$ radicals reacted with the $\mathrm{Si}$ surface to form the carbonized layer. DMGe is an effective carbon source to form $\beta$-SiC, because a clean Si surface can be carbonized even at temperatures as low as $650^{\circ} \mathrm{C}$, and it gives better surface morphology after longer exposure. In the subsequent epitaxial growth on the carbonized layers, the precise control of $\mathrm{Si}_{2} \mathrm{H}_{6}$ and $\mathrm{C}_{3} \mathrm{H}_{8}$ supply resulted in epitaxial layers with good crystallinity and a smooth surface at $1000^{\circ} \mathrm{C}$. The good 
crystallinity of the $\beta$-SiC layer with single-domain $(3 \times 2)$ structure could also be obtained by an optimum $\mathrm{Si}_{2} \mathrm{H}_{6} / \mathrm{DMGe}$ flow rate at a temperature as low as $910^{\circ} \mathrm{C}$.

\subsection{Use of C- and Si- Containing Precursors}

Most $\beta$-SiC growth proceeds through the use of separate precursors for $\mathrm{Si}\left(\mathrm{SiH}_{4}, \mathrm{Si}_{2} \mathrm{H}_{6}\right.$, or $\left.\mathrm{SiH}_{2} \mathrm{Cl}_{2}\right)$ and carbon $\left(\mathrm{C}_{2} \mathrm{H}_{2}, \mathrm{CH}_{4}\right.$, or $\left.\mathrm{C}_{3} \mathrm{H}_{8}\right)$. Recently there have been some reports on attempts to grow $\mathrm{SiC}$ at lower deposition temperatures by using a single $\mathrm{C}$ - and $\mathrm{Si}$ - containing molecular precursor. Examples, including alkylsilanes, such as hexamethyldisilane, ${ }^{40}$ methylsilane, ${ }^{41}$ trimethylsilane, $^{42}$ and t-butyldimethylsilane, ${ }^{43}$ have all produced single crystalline layers of high quality $\mathrm{SiC}$ at atmospheric pressure or low pressure ( $<5$ torr) for temperature above $1000^{\circ} \mathrm{C}$. In these conventional CVD methods, however, growth below $1000^{\circ} \mathrm{C}$ resulted in unwanted incorporation of Si microcrystals into the films. The growth of high quality $\mathrm{SiC}$ at lower temperature, therefore, has not been demonstrated for the organosilicon growth species.

GSMBE using organo-silicon species may be a viable option for $\beta$-SiC growth at lower temperatures than chemical vapor deposition using aforementioned alkylsilanes in formation of $\mathrm{Si}-\mathrm{C}$ bonds in the film. Nakazawa et al. are believed to be the only group that have done extensive research on this particular subject. ${ }^{44,45}$ In their work, methylsilane $\left(\mathrm{CH}_{3}-\mathrm{SiH}_{3}\right)$ was the choice of source gas due to the fact is has the same stoichiometry $(1: 1)$ as SiC. These authors studied the GSMBE of $\beta$-SiC on $\mathrm{Si}(100)$ and the relation between the growth parameters (temperature and MS partial pressure) and the quality of the SiC films. They concluded that there existed a certain value for each of the growth parameters that optimizes the film quality and the optimum growth temperature appeared to decrease with decreasing MS partial pressure. 
Using an MS pressure of $5.0 \times 10^{-5}$, high quality $3 \mathrm{C}$-SiC films on $\mathrm{Si}(100)$ were grown at the a temperature of as low as $900^{\circ} \mathrm{C}$.

\subsection{Studies of $\beta-S i C$ Growth at WVU}

Lannon studied the thermal interaction of ethylene $\left(\mathrm{C}_{2} \mathrm{H}_{4}\right)$ on $\mathrm{Si}(100) 2 \times 1$ surface. The films formed at $1000 \mathrm{~K}$ were composed of a mixture of $\mathrm{SiC}, \mathrm{sp}^{2}-\mathrm{C}$ (graphite) and $\mathrm{sp}^{3}-\mathrm{C}$ (diamond). Elemental Si-LMM was present on the surface throughout the growth process and acted as a surfactant to "maintain the reactivity of the surface and minimize the surface free energy." It was suggested that Si segregation and diffusion toward the surface were the primary means of supporting continued growth in the absence of a gas phase Si-containing growth species in $\mathrm{SiC}$ formation. ${ }^{46}$

Gold grew thin $\mathrm{SiC}$ films on $\mathrm{Si}(100) 2 \times 1$ substrates using a methyl $\left(\mathrm{CH}_{3}\right)$ radical source. At low temperature $\left(652^{\circ} \mathrm{C}\right)$, the near surface layers were depleted in $\mathrm{Si}$ and a "segregated Siadlayer" was present on the surface. Films grown at high temperature $\left(727^{\circ} \mathrm{C}\right)$ exhibited a "SiC growth window during which only SiC was formed" and continued growth after the induction resulted in formation of an $\mathrm{sp}^{3}-\mathrm{C}$ (carbon-rich) film with no segregated Si ad-layer. ${ }^{47}$

The surface chemical activity contributes greatly to the overall quality of $\beta$-SiC growth. Si atoms originating from the substrate diffuse through the growing film or more likely along grain boundaries, which often results in defects in crystal formation and affects the chemical reaction. Most recently, Ziemer conducted research on $\mathrm{SiC}$ thin film growth characteristics using GSMBE in relation to the initial state of surface, growth temperature, flux, and growth species. $^{48}$ It was concluded that the starting-surface conditions had more effect on the characteristics of the film than any other growth condition. In particular, three surfaces were 
studied. The first was a hydrogen-terminated Si (100) $1 \times 1$ produced by the so-called "Fenner" wet chemical etch. The second was the "bare" Si (100) $2 \times 1$ produced by annealing the Fenner etched surface, and thirdly by Ar-ion etching and annealing of the Si (100) surface. It was found that reaction of the H-terminated $\mathrm{Si}(100) 1 \times 1$ with a hydrocarbon resulted in a very effective $\mathrm{Si}$ diffusion barrier.

In addition to studies on the effect of surface pretreatment, Ziemer also investigated conversion layer and thin film growth using trimethylsilane (TMS). The high flux case TMS clearly enhanced the growth of a carbon rich film suggesting the possibility that using a different silicon-carbon ratio in single precursor could point to better composition.

\subsection{Bulk Growth of $\alpha-S i C$}

The first known crystal growth of $\mathrm{SiC}$ was done by Edward G. Archeson in 1884 . He heated a mixture of clay and coke in an iron bowl electric furnace with a carbon arc light and found silicon carbide crystal attached to the carbon electrode. ${ }^{49}$ It was then known as carborundum, and found an important industrial application in abrasives. Around the same time, Henri Moissan in France produced a similar compound from a mixture of quartz and carbon. For this reason, some mineralogists still refer to silicon carbide as moissanite.

Bulk crystalline $\mathrm{SiC}$ is generally prepared using the patented Lely method ${ }^{50}$, also commonly referred to as physical or sublimation vapor transport method. (It's not possible to grow $\mathrm{SiC}$ crystals from the melt as $\mathrm{SiC}$ does not melt. Instead, it decomposes at temperatures of about $2830^{\circ} \mathrm{C}$.) Here synthesized $\mathrm{SiC}$ powder is evaporated at $2500^{\circ} \mathrm{C}$ in graphite crucibles in an argon atmosphere under essentially isothermal conditions. Under these conditions platelets were found to nucleate randomly along vapor transport flow paths into the cavities. While grown junction and diffused junction devices have been fabricated using these platelets, they 
generally displayed non-uniform physical and electrical properties, and their size and random shapes made them difficult to handle. ${ }^{51}$ Later advances in the growth of single crystal boules of $\mathrm{SiC}$ is due to the work of Tairov and Tsvetkov ${ }^{52}$. Here $\mathrm{SiC}$ vapor was transported under near vacuum conditions from a subliming $\mathrm{SiC}$ source to seed crystal located in the low-temperature zone of the furnace, where a $6 \mathrm{H}-\mathrm{SiC}$ boule $8 \mathrm{~mm}$ in diameter and $8 \mathrm{~mm}$ long was grown. This method, commonly known as the Modified Lely Method, was the first viable growth method to make the resulting single crystal $\mathrm{SiC}$ commercially interesting. In 1987, the first commercial $\mathrm{SiC}$ substrate supplier, CREE, was founded. The single crystal growth technology has been continuously refined to reduce defect density and increase wafer size. Single-crystal wafer with diameter up to 3 inches and average micropipe density (MPD) less than $50 \mathrm{~cm}^{-2}$ can be purchased for several thousand dollars. (Price depends on doping type, wafer grade, micropipe density, et al.) Crack-free, fully single-crystal 4-inch $6 \mathrm{H}$ and $4 \mathrm{H}$ wafers have been demonstrated at the R\&D level. ${ }^{54}$ However, as crystals grow to become even larger diameter boules, threading dislocation and micropipes develop in the lateral growth as well as c-axis growth. Methods of controlling such defects were studied and the most important parameter for enhancing radial expansion growth quality was believed to be the radial temperature gradient. ${ }^{55}$

\subsection{SiC Etching Studies}

Commercially available $\mathrm{SiC}$ substrates are generally of $4 \mathrm{H}$ or $6 \mathrm{H}$ polytypes. They are grown as a 2 -inch or 3 -inch boules, which are sliced into wafers hundreds of microns in thickness. To produce a smooth surface for electronic applications and further epitaxial growth, the manufactures polish these wafers using fine-grit diamond "sandpapers". Due to its extreme hardness (9.5 on Rockwell hardness scale), the wafers are still left with fine scratches. Although 
invisible to the naked eye, these scratches can be detrimental to electronic applications and epitaxial layers growth alike.

In order to remove the damaged $\mathrm{SiC}$ layer caused by polishing process, a molten salt etching method involving $\mathrm{NaOH}$ or $\mathrm{KOH}$ was used most conventionally, and studied by several investigators. ${ }^{56,57,}$ Molten salt etching is a combination of thermal and chemical etching techniques. It takes advantage of the higher internal energy associated at the material's grain boundary. At the elevated temperature of molten salts, the higher energy at the grain boundaries is relieved, producing a rounded grain boundary edge which can be observed by optical or electron microscope techniques. Unfortunately, the molten salt etch method was very aggressive; etch pits were formed on the surface. Brander and Boughey experimented with an anodic etch using HF solutions at room temperature, but the method can only be used satisfactorily on p-type material. ${ }^{58}$ Gulbransen and co-workers investigated the oxidation of silicon carbide at $1150^{\circ}$ to $1400^{\circ} \mathrm{C}$ and at $9 \times 10^{-3}$ to $5 \times 10^{-1}$ torr oxygen pressure. ${ }^{59}$ Using this method, volatile silicon monoxide, $\mathrm{SiO}(\mathrm{g})$, was formed along with $\mathrm{CO}, \mathrm{CO}_{2}$, and a small amount of $\mathrm{SiO}_{2}$, thus it was not favorable for obtaining a clean surface.

The gas phase hydrogen etching method was first reported by Chu in $1965^{60}$, with an etch rate of $0.3-4 \mu \mathrm{m} / \mathrm{min}$ at a temperature range of $1600^{\circ} \mathrm{C}$ to $1750^{\circ} \mathrm{C}$. The linear hydrogen gas velocity is approximately $8.5 \mathrm{~cm} / \mathrm{sec}$. This method provided a smooth and clean etched surface with a very simple operation, and the crystal growth can be carried out successively after etching. In 1966, Kumagawa et al. ${ }^{61}$ did a more comprehensive study on hydrogen etching of $\mathrm{n}$ doped $6 \mathrm{H}-\mathrm{SiC}$ at $1700^{\circ} \mathrm{C}$ to $2000^{\circ} \mathrm{C}$ with varying flow velocities $(2 \sim 6 \mathrm{~cm} / \mathrm{sec})$ and hydrogen partial pressure $(0.25 \sim 1 \mathrm{~atm})$. The etch rate $(0.5$ up to $5 \mu \mathrm{m} / \mathrm{min})$ was found to increase with each of these three variables. In the following decades, many variations of this high temperature 
hydrogen etch study were done including the use of forming gases. Modrak et al. experimented with different susceptor materials and found etch rate varied with them; ${ }^{62}$ Saidov et al. used forming gas of $\mathrm{H}_{2}+\mathrm{CH}_{3} \mathrm{SiCl}_{3}$, and learned the effect of $\mathrm{H}_{2}$ flow velocity and etching temperature was consistent with Kumagawa's early findings ${ }^{63}$ Karlsson and Nordell used $\mathrm{H}_{2}, \mathrm{H}_{2}$ $+\mathrm{C}_{3} \mathrm{H}_{8}$, and $\mathrm{H}_{2}+\mathrm{HCl}$ for in-situ etches and found the last mixture had the most aggressive etch rate though all three produced similar surface roughness; ${ }^{64}$ and most recently, Ramachandran et al. etched with pure hydrogen at atmospheric pressure and temperatures of $1600-1700^{\circ} \mathrm{C}$ (attained on a tantalum strip heater) and were able to obtain flat terraces $1.5 \mathrm{~nm}$ high and hundreds of nm wide. ${ }^{65}$

\subsection{The Goal of Research}

The goal of this research is to develop novel methods of growing $\mathrm{SiC}$ films on $1 \times 1$ $\mathrm{Si}(001)$ and hydrogen-etched $6 \mathrm{H}-\mathrm{SiC}$ substrates.

Based on the research described in the literature, the present studies extend previous heteroepitaxial $\mathrm{SiC}$ growth studies using TMS at WVU to dimethylsilane (DMS) and methylsilane (MS). In the second part of this study, the effect of the high temperature hydrogen etch will be examined and optimized to create large, flat, and regular-height terraces as described by Ramachandran. The final portion of the study involves the homoepitaxial growth of $\mathrm{SiC}$ thin films as prepared by hydrogen-etch in attempt to produce a larger, flat surface on the substrate using the gas-source precursors DMS and MS. 


\section{Chapter 3: Experimental Approach}

\subsection{Growth and Analysis Systems}

Figure 3.1 shows a schematic of the apparatus used in this research. It consisted of UHV growth and analysis chambers, and a loading chamber that was used for introducing the samples without breaking vacuum in the UHV chambers. The chambers were constructed from stainless steel and isolated by UHV gate valves to prevent cross-contamination. Samples were transferred between chambers using a magnetically coupled linear transfer without breaking vacuum. The growth chamber was equipped with two turbo molecular pumps, a Leybold Turbotronik NT 150/360 and a Varian Turbo-V 550, which provided 400L/s and 550L/s of pumping speed, respectively. These were backed by an Alcatel 2012A direct drive vacuum pump. The base pressure in the growth chamber was $\sim 5 \times 10^{-10}$ torr. The analysis chamber was pumped by a Perkin Elmer TNB X3 ion pump and had a base pressure of $\sim 5 \times 10^{-10}$ torr. The loading chamber was pumped by a Pfeiffer TCP-015 turbo molecular pumping station and typically reached a pressure of $<10^{-5}$ torr in 40 minutes.

The UHV base pressure allows pristine, controlled starting surfaces for film growth because of the lack of background gas-surface collisions. The flux at which gas phase molecules strike a surface at $10^{-10}$ Torr is approximately $10^{11}$ atoms $/ \mathrm{cm}^{2} \cdot \mathrm{s}$, or about $10^{-4} \mathrm{ML} / \mathrm{s}$ (monolayers per second) where a ML is approximated as $10^{15}$ atoms $/ \mathrm{cm}^{2}$. 


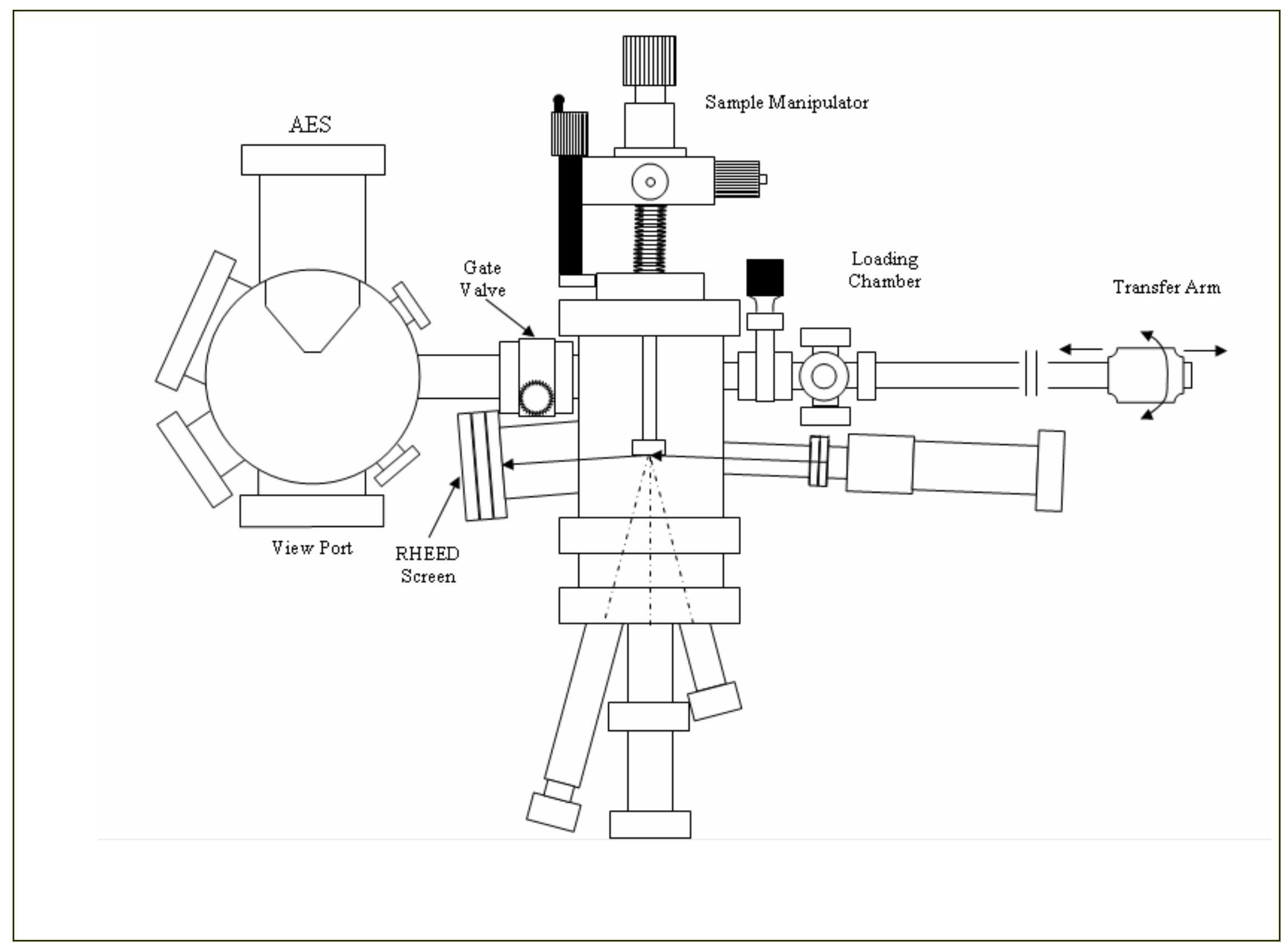

Figure 3-1. Gas Source MBE system at Material and Surface Studies Laboratory at WVU. 
Sample manipulation in the growth chamber was accomplished via a Fisions DPRF-25 x, $\mathrm{y}, \mathrm{z}$ rotary motion feed through. The sample mounting block attached to this feed through allowed for in-vacuum mounting and heating as described later. A similar arrangement (without heating apparatus) was provided in the analysis chamber.

A magnetically driven linear transfer arm was used to move samples between the loading, growth, and analysis chamber. Using a twist-to-lock and twist-to-unlock design, the sample mounting block, commonly referred to as "puck", could be locked onto or unlocked from the sample transfer block or receiver that was located on the end of the transfer arm. A photo of the puck and receiver is shown in Figure 3-2.

The puck was equipped with two sets of pins ( 3 pins in each set). The front set locks into the transfer block, while the second set locks into a similar receiver attached to the $\mathrm{x}, \mathrm{y}, \mathrm{z}$ rotary motion feed-through in the analysis or the growth chambers. The sample transfer process in the growth chamber required that the puck and transfer receiver be flipped perpendicular to the axis

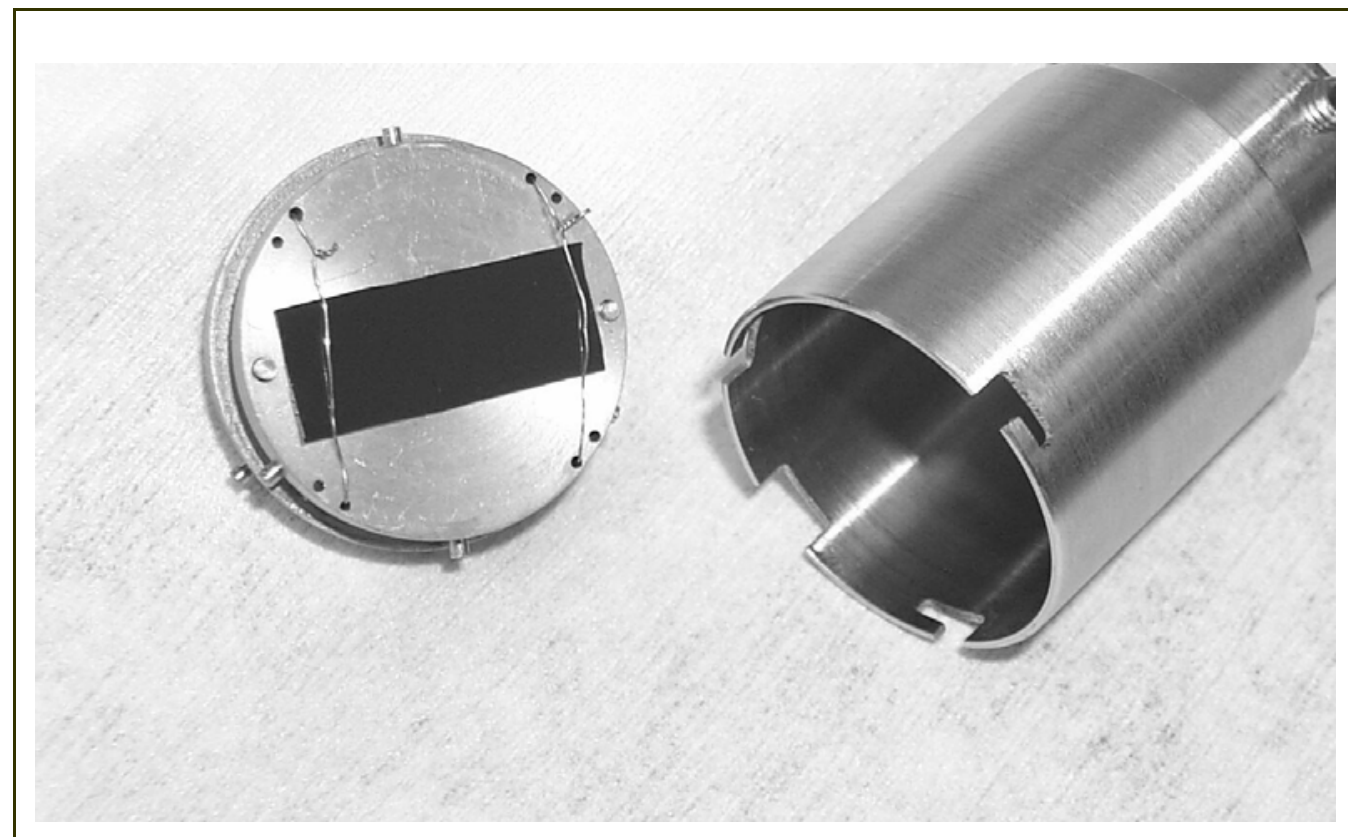

Figure 3-2. Sample holder with sample and notched end of receiver. (Courtesy: K. Ziemer) 
of the transfer arm. This was accomplished using a $90^{\circ}$ swivel that was also designed and constructed here at WVU.

The sample block mounted in the growth chamber was equipped with a resistive heater assembly described in detail by Ziemer. ${ }^{48}$ This allowed the temperature to be maintained in the range of up to $1000^{\circ} \mathrm{C}$ as monitored by a spring mounted K-type thermocouple in contact with the back of the puck. High temperature was obtained via resistive heating of tantalum wire and the current was controlled by a Eurotherm Model $825 \mathrm{~S}$ temperature controller and powered by a Eurotherm Model 832 Silicon Crystal Rectifier (SCR) power supply.

\subsubsection{Sample Surface Temperature Measurement}

To obtain a more accurate measurement of the front face temperature of the sample, an Omega OS1562 Fiber Optic Infrared Sensor was used as a non-contact measuring device. Even though the "puck" is made of molybdenum, an excellent heat-conducting material, there was still a significant temperature gradient of $200^{\circ} \mathrm{C}$ or more between the substrate surface (as measured by the infrared sensor) and back face (as measured by the thermocouple) under growth conditions. The infrared measurement was adjusted according to the emissivity of $\mathrm{Si}(\varepsilon=0.64)$ or $\mathrm{SiC}(\varepsilon=0.86)$, and it was the actual growth temperature. See Appendix A for details on the experimental procedures and data used for substrate emissivity calculation.

\subsection{Gas Sources}

DMS was transferred to small stainless steel cylinders (Whitey SS-4CD-TW-SO), and MS was kept in the original container provided by Gelest. Both were fitted with bellow-sealed valves and VCR connectors that were then connected to the source gas inlet system. The entire gas manifold is shown in Figure 3-3. A Varian Model 203 variable leak valve was gradually 


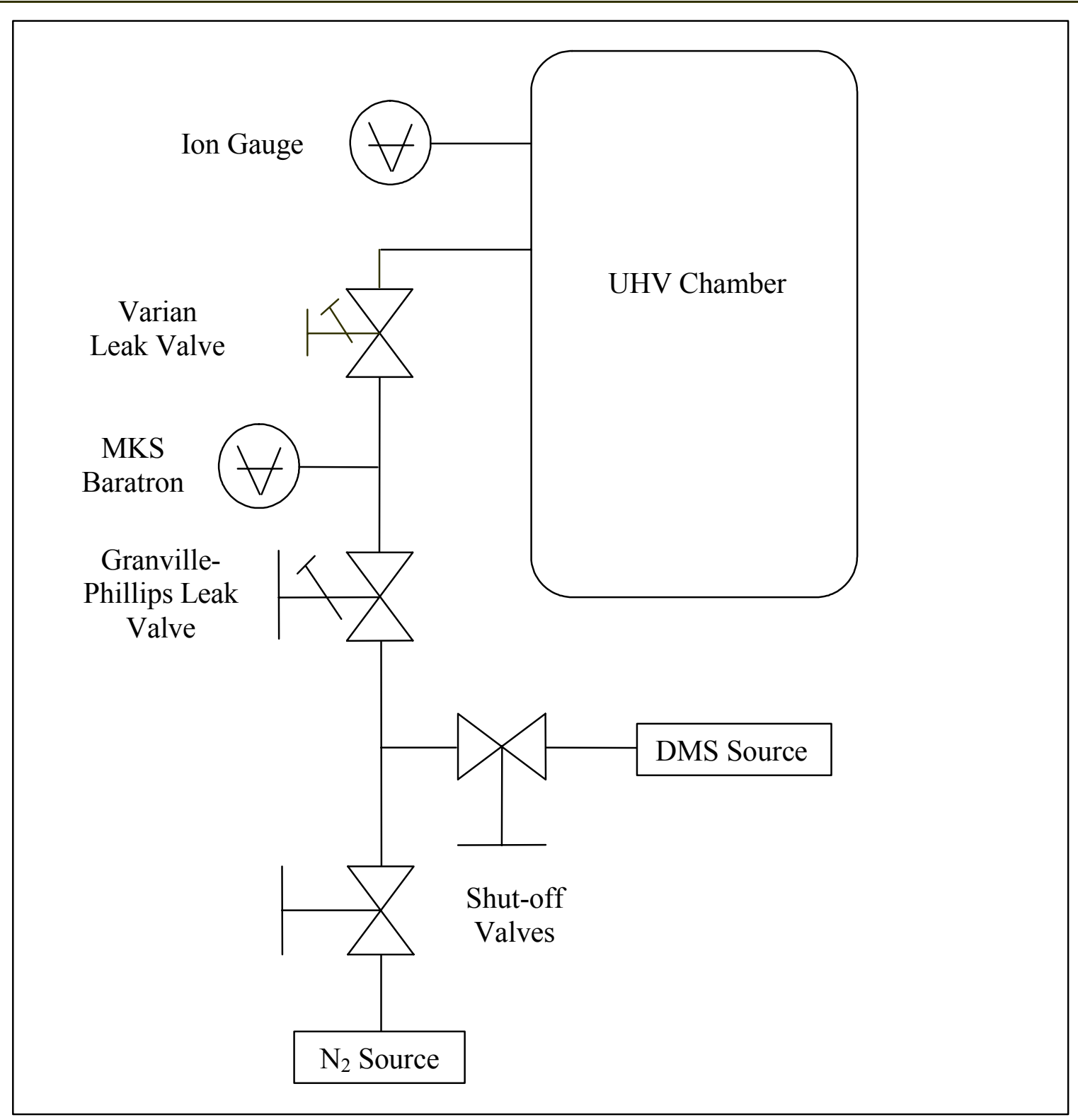

Figure 3-3. Growth chamber gas manifold diagram.

opened, allowing gas to effuse through a small orifice in the valve. An ion gauge located in growth chamber was used to monitor the working pressure of the growth chamber.

The ion gauge accomplished pressure measurement through ionizing a fraction of the gas molecules and measuring the resulting ion current. As supplied by the vendor, these gauges are calibrated to correctly read the $\mathrm{N}_{2}$ (or air) pressure in the chamber. Since ionization efficiency is 
different for different gas molecules, the observed pressure for gases other than $\mathrm{N}_{2}$ must be corrected using an "ion gauge sensitivity factor". To establish this factor, the leak valve was opened to begin a DMS or $\mathrm{N}_{2}$ flow through the system. The throughput (proportional to the molar flow rate) is given by the equation

$$
\dot{V} P_{c}=\frac{\pi \cdot d^{2}}{4} \sqrt{\frac{k T}{2 \pi \cdot m}} P_{o},
$$

where

$\mathrm{P}_{\mathrm{c}}=$ true pressure inside chamber $<$ torr $>$

$\mathrm{P}_{\mathrm{o}}=$ pressure behind the orifice $<$ torr $>$

$\mathrm{d}=$ diameter of diffusion orifice $<\mathrm{cm}>$

$\mathrm{m}=$ mass of a single gas molecule

$\mathrm{k}=$ Boltzmann's constant $=1.4 \times 10^{-16}<\mathrm{erg} /{ }^{\circ} \mathrm{K}>$

$\mathrm{T}=$ temperature $<{ }^{\circ} \mathrm{K}>$

$\dot{V}=$ growth chamber turbo-molecular pumping speed $<1000 \mathrm{~L} / \mathrm{s}$. $>$

The pressure, $\mathrm{P}_{\mathrm{o}}$, behind the orifice was measured with an MKS Model KDR-D-1 0-10 Torr Baratron. The Baratron pressure measurement was independent of the type of gas. The pressure, $\mathrm{P}_{\mathrm{mc}}$, measured in the chamber using the ion gauge, depended on the gauge sensitivity factor, $\varphi$, for the gas. Specifically, $\mathrm{P}_{\mathrm{mc}}$, is related to the true chamber pressure, $\mathrm{P}_{\mathrm{c}}$, by the relationship

$$
P_{c}=\varphi P_{m c} .
$$

Combined with the previous equation,

$$
\varphi P_{m c}=\frac{\pi \cdot d^{2}}{4 \dot{V}} \sqrt{\frac{k T}{2 \pi \cdot m}} P_{o} .
$$


For $\mathrm{N}_{2}, \varphi=1$, so

$$
P_{m c N_{2}}=\left(\frac{\pi \cdot d^{2}}{4 \dot{V}} \sqrt{\frac{k T}{2 \pi \cdot m_{N_{2}}}}\right) P_{o N_{2}}=C_{N_{2}} P_{o N_{2}}
$$

Terms in side the parenthesis remain same for the same gas and their product is a constant and designated as $C_{\mathrm{N}_{2}}$ here. Thus, using $\mathrm{N}_{2}$ as the source gas, a plot of $P_{\text {mcN }_{2}}$ vs. $P_{\mathrm{ON}_{2}}$ gives $C_{\mathrm{N}_{2}}$ as the slope.

Likewise, for an unknown gas x, $P_{m c x}=\frac{C_{x}}{\varphi_{x}} P_{o x}$.

Since $C_{x}=\sqrt{\frac{m_{N_{2}}}{m_{x}}} C_{N_{2}}$, the chamber pressure for gas $\mathrm{x}$, as displayed by ion gauge, is

$$
P_{m c x}=\frac{1}{\varphi_{x}} \sqrt{\frac{m_{N_{2}}}{m_{x}}} C_{N_{2}} P_{o x}
$$

Thus a plot of $P_{m c x}$ vs. $P_{o x}$ allowed $\varphi_{x}$ to be determined. Figure 3-4 shows such a plot for DMS gas, which has a sensitivity factor of 0.25 . Similar experiments determined the sensitivity factor for MS to be 0.83 .

Just prior to the beginning of the growth process, the DMS pressure the chamber was slowly increased to desired level $\left(\sim 10^{-5}\right.$ Torr $)$ by adjusting the variable leak valve. This method of exposing the substrate to the reactant gas molecules randomly is called "backfilling", and it contrasts with the molecular beam method.

Once the pressure of the backfilled reactant gas was steady, the substrate temperature was quickly ramped $\left(\sim 100^{\circ} \mathrm{C} /\right.$ minute $)$ to the desired growth temperature. Substrate heating and temperature measurement have been described in detail by Ziemer ${ }^{48}$ and will not be repeated here. Temperatures used in these experiments were $700^{\circ} \mathrm{C}$ and $800^{\circ} \mathrm{C}$. These were the front face 


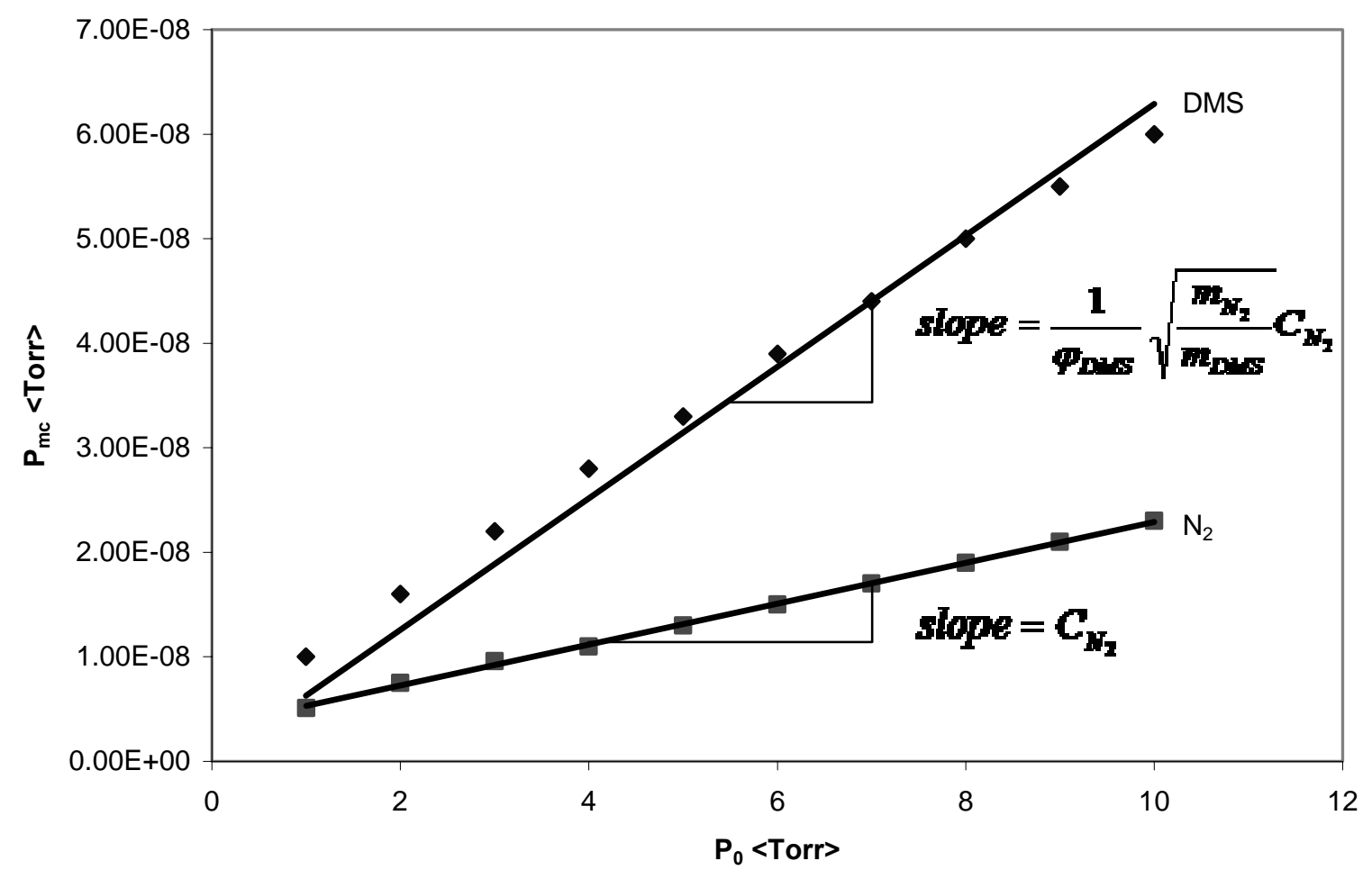

Figure 3-4. DMS ion gauge factor calculation plot.

temperatures based on Ziemer's analysis and were accurate to $\pm 16^{\circ} \mathrm{C}$ in absolute temperature. The day-to-day variations were very low, meaning high consistencies in actual growth temperature could be achieved.

The flux of molecules hitting the substrate surface during the growth process can be determined using the equation

$$
J=\frac{P}{\sqrt{2 \pi \cdot m \cdot k \cdot T}},
$$

where

$\mathrm{P}=$ pressure of reactant inside the chamber (Torr)

$\mathrm{m}=$ mass of one reactant gas molecule $(\mathrm{kg})$

$\mathrm{k}=$ Boltzmann's constant $=1.38 \times 10^{-23} \mathrm{~m}^{2} \mathrm{~kg} \mathrm{~s}^{-2} \mathrm{~K}^{-1}$ 
$\mathrm{T}=$ temperature in ${ }^{\circ} \mathrm{K}$ (taken as $\left.300^{\circ} \mathrm{K}\right)$.

The normal DMS molecular fluxes used in these experiments were $0.2 \mathrm{ML} / \mathrm{second}$ and 1.9 ML/second. A ML (monolayer) corresponds to $10^{15} / \mathrm{cm}^{2} \cdot \mathrm{sec}$.

\subsection{High Temperature Hydrogen Furnace}

The high temperature furnace was a Model 1730-12HT made by CM Furnaces. (Figure 3-5) It was a laboratory scale tube-and-box furnace with a maximum operating temperature of $1700^{\circ} \mathrm{C}$ in air. The furnace used high purity alumina fiber insulation and Kanthal molydisilicide heating elements. A separate control unit was provided, and it acted as a power supply unit, source gases flow rate controller, and heating program controller used in conjunction with a type "B" thermocouple.

One tank of ultra high purity argon, which was the carrier gas for hydrogen, and one tank of ultra high purity hydrogen were connected to the gas source inlets on the control unit using $1 / 4^{\prime \prime}$

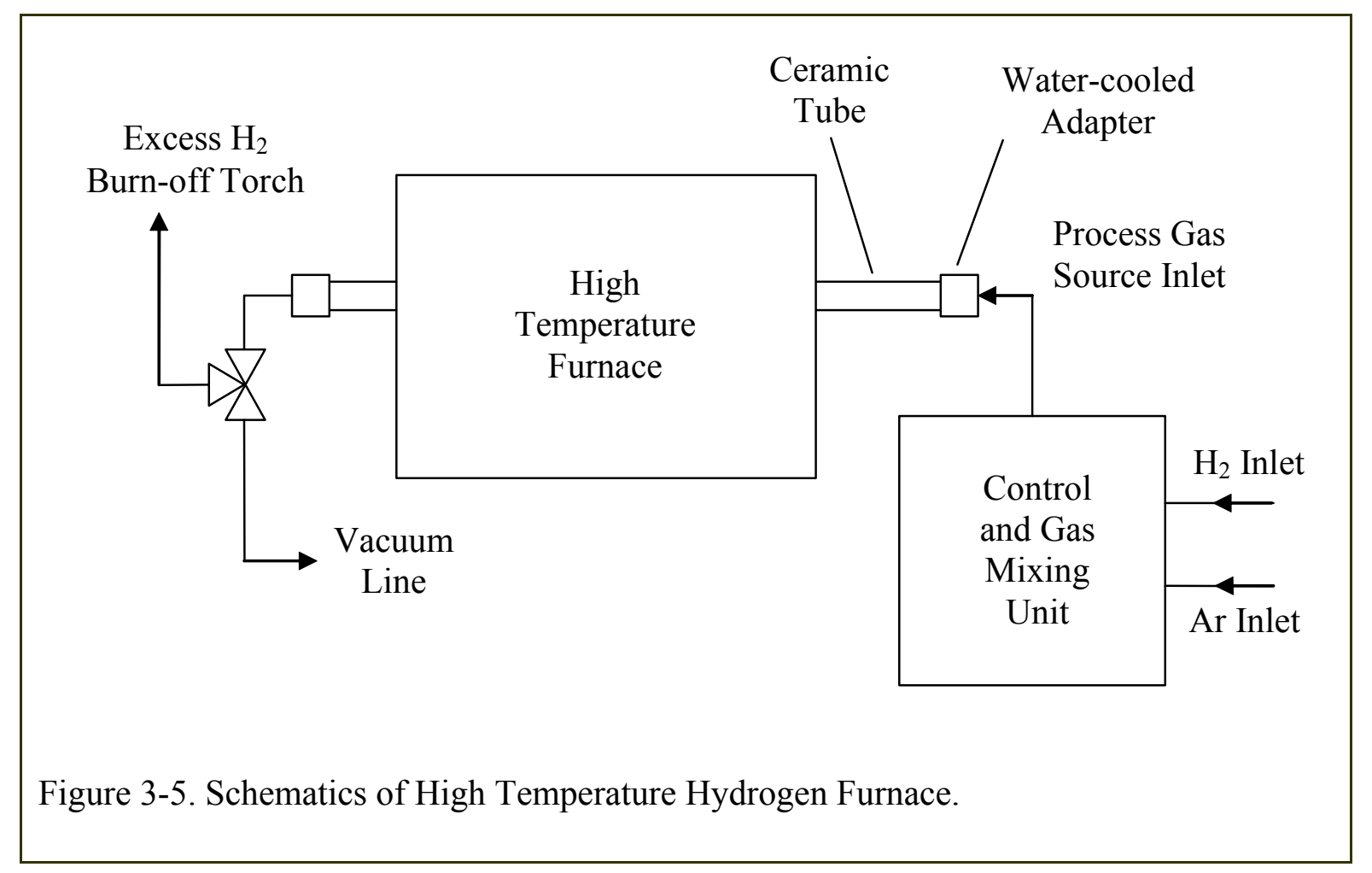


stainless steel piping. Check valves were placed on both lines to prevent unwanted backflow. Two Fisher \& Porter Model 10A6130 rotameters installed on the front face of the control unit were used to adjust for the proper rate (pre-calibrated for up to 8.0 standard cubic feet per hour for $\mathrm{Ar}$ and up to $8.4 \mathrm{SCFH}$ for $\mathrm{H}_{2}$ ) and mixture of source gases. This typical flow rate of forming gas ( $8 \mathrm{SCFH}$ Ar with $5 \% \mathrm{H}_{2}$ ) was equivalent to $8 \mathrm{~cm} / \mathrm{sec}$ across the surface of the sample.

In using hydrogen, its flammability and explosiveness are major safety concerns. Multiple steps were implemented for leak detection, prevention, and excessive gas removal. The entire furnace, with exception of the control unit, was placed inside venting hoods, where the increased ventilation rate ensured proper removal of any residual hydrogen. An excess $\mathrm{H}_{2}$ burnoff torch was provided and placed at the exhaust end of the tube. Two igniters (a primary and a back-up) placed in the torch ignited automatically upon the start of heating programs and burned off excessive hydrogen. A yellow flame was clearly visible when the flow rate was high enough. The UHP hydrogen gas tank was fitted with a Matheson Model 6104 flash arrester and placed away from the furnace. A gas detector by Gastronics capable of detecting the presence of both argon and hydrogen was used before and during the experiment at the high-risk leaking spots (adaptors, Swagelok connectors, etc.) The 11/2" OD ceramic tube, which was fitted with a Cajon Ultra-Torr $^{\circledR}$ SS-24-UT-S-24 stainless steel adapter, replaced the less reliable strap-and-clamp type seal originally provided by CM Furnaces. The adapter featured a Viton o-ring sealant that could be connected and disconnected repeatedly without affecting seal performance. Since these adaptors and o-rings were only rated for up to $200^{\circ} \mathrm{C}$, water-cooling jackets were made for each adapter to prevent o-ring degradation even though a thermocouple placed onto the sections of tube immediately before the adapters indicated that the temperature never exceed $100^{\circ} \mathrm{C}$ in those 
areas. Finally, a Whitney three-way gas valve was installed on the outlet side of the tube. At the up position, it allowed gas to pass through and into the excess hydrogen burn-off torch. At the down position, it sealed off the tube from the atmosphere and connected it to a vacuum line. This setting enabled the tube to be pumped down, and the level of vacuum achieved as shown by a Baratron readout was a good indicator of whether the tube achieved a sufficient level of seal.

Heating control was carried out by a Eurotherm 2404 Controller Setpoint Programmer. A heating programs consist of segments that perform tasks such as Ramp (up or down), Dwell, Step, Call (another program), and End. Target heating rate and temperature could also be reached via manual control of heating current, but programming control improves system damping and was the easier method for controlling heating rate. The sample was usually ramped up to $1500-1600^{\circ} \mathrm{C}$ at a pre-determined rate $\left(400^{\circ} \mathrm{C} / \mathrm{hr} \text { up to } 1000^{\circ} \mathrm{C} \text { and } 300^{\circ} \mathrm{C} / \mathrm{hr} \text { thereafter }\right)^{1}$ and maintained for a dwell time of 0 to several hours at the etching temperature. Finally, it is ramped down to room temperature at the same rates.

\subsection{Analytical Methods}

\subsubsection{Auger Electron Spectroscopy (AES)}

This analysis tool, as equipment in our laboratory, had been described in detail by Ziemer $^{48}$, and the discussion presented here draws heavily from her work.

Auger Electron Spectroscopy is a surface specific technique that probes the electronic energy levels of atoms using electron beam stimulation utilizing the emission of such electrons. It is one of the commonly employed methods in the examination of material surface chemical analysis. Auger spectroscopy can be considered as involving three basic steps: (1) Atomic

\footnotetext{
${ }^{1}$ The temperature ramp rates are determined by safety consideration based on the thermal expansion characteristics of the alumina tube per information provided by Coorstek.
} 


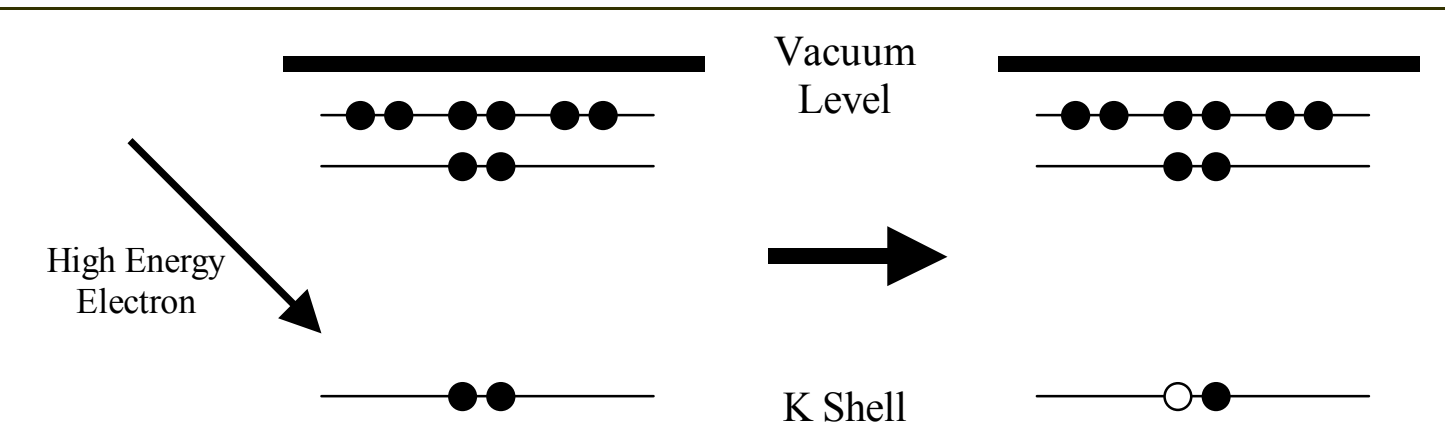

Figure 3-6. AES incident electron beam creates a hole at the core level.

ionization (by removal of a core electron); (2) Electron emission (the Auger process); (3) Analysis of the emitted Auger electrons. The Auger process is initiated by creation of a core hole - accomplished by exposing the sample to a beam of high energy electrons in the range 2 $10 \mathrm{keV} \cdot{ }^{66}$ (Figure 3-6)

The ionized atom is now at an excited state and rapidly relaxes back to a lower energy state by Auger emission during which one electron falls from a higher level to fill the initial core hole. The second electron (the Auger electron) is emitted simultaneously by the energy released

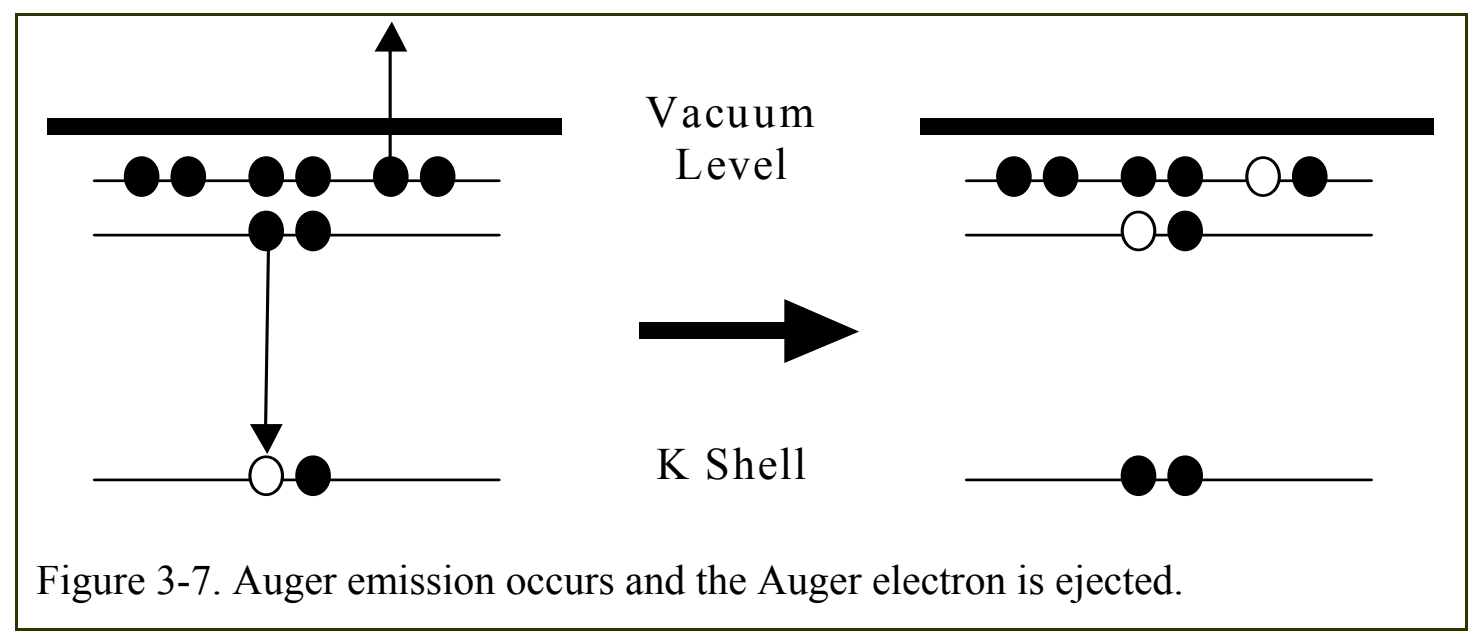

in this process. Part of this energy overcomes the binding energy while the Auger electron retains the remainder as its kinetic energy. (Figure 3-7) 
The depth of excitation into the surface depends on the energy of the incident beam, but most of the Auger electrons originate within the first few atomic layers of the surface. (Once again, the importance of minimal contamination is validated here since the technique is so sensitive to the surface conditions.)

AES spectra were collected using a 15-110A model single-pass analyzer scanning Auger system by Physical Electronics Industries, Incorporated. The instrument operated with a $2 \mathrm{kV}$ electron beam energy, a $1.0 \mu \mathrm{A}$ beam current, and is aligned elastically at $2000 \mathrm{eV}$.

AES analysis of these emitted electrons measures the energy distribution $N(E)$ of the Auger electron as a function of the electron energy E. The position and shape of the Auger peaks identifies individual elements and chemical information. The spectrum is generally

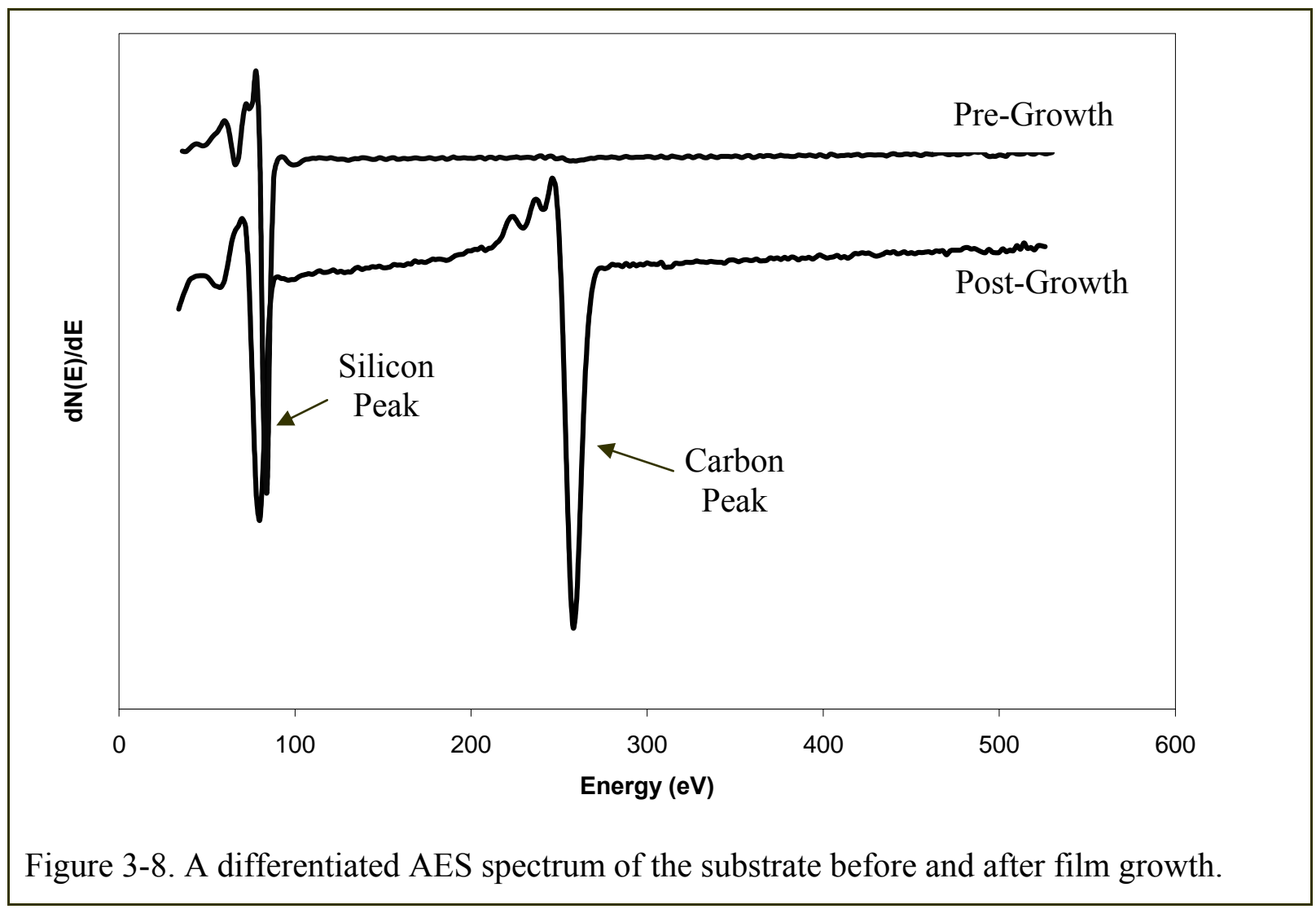


viewed in its differentiated form so peak shape and size are more distinct.

Figures 3-8 shows a typical differentiated spectrum of a substrate prepared using Fenner etch and the corresponding differentiated spectrum of the substrate after film growth.

To analyze the Auger spectra, it is necessary to know the composition and the relative carbon-KLL intensity of each film grown for better understanding the growth mechanisms. KLL for $\mathrm{C}$ (or LMM for $\mathrm{Si}$ ) refers to the particular orbitals involved in Auger electron transitions. The C-KLL intensity can be further de-convoluted into three possible bonds of SiC, graphite

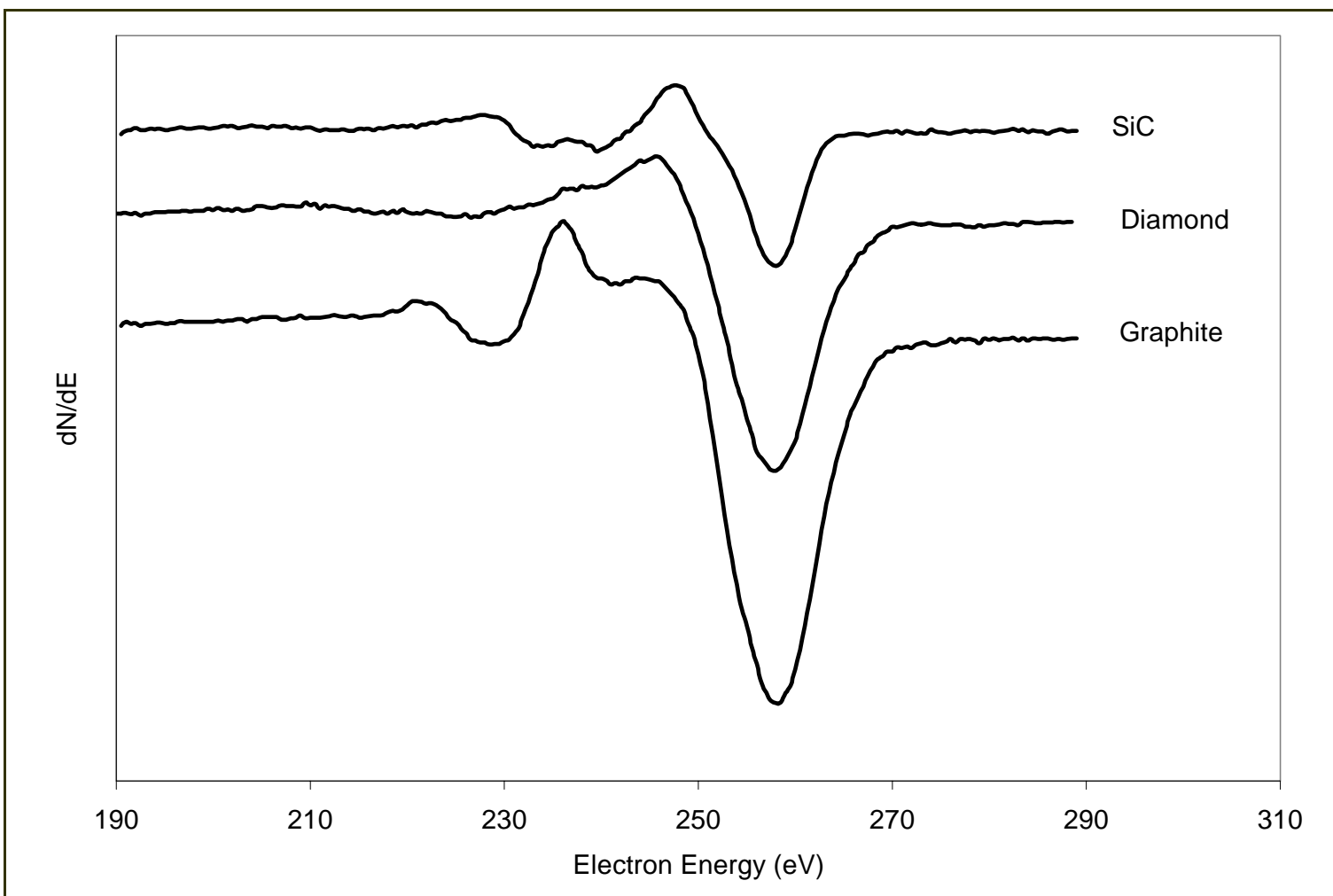

Figure 3-9. Differentiated AES spectra of the SiC, diamond, and graphite standards.

( $\mathrm{sp}^{2}$-hybridized bonds), and diamond ( $\mathrm{sp}^{3}$-hybridized bonds) through factor analysis since the Auger transition is sensitive to the bonding environment. Figure 3-9 shows the differentiated CKLL scans of $\mathrm{SiC}, \mathrm{sp}^{2}$, and $\mathrm{sp}^{3}$ standards. 


\subsubsection{AES General Intensity Equation}

The total intensity of an elemental AES peak, $\mathrm{I}_{\mathrm{i}}$, is determined as

$$
I=A \cdot I_{p} \cdot X_{i} \cdot N_{i} \cdot \beta_{i} \cdot \Phi_{i} \cdot \Psi_{i} \cdot R \cdot T_{i} \cdot \lambda_{i} \cdot \cos \theta
$$

where

$$
\begin{aligned}
& A=\text { surface area analyzed } \\
& \mathrm{I}_{\mathrm{p}}=\text { primary beam current } \\
& \mathrm{X}_{\mathrm{i}}=\text { fraction of atoms participating in the Auger signal } \\
& \mathrm{N}_{\mathrm{i}}=\text { number density of atoms of } \mathrm{i}^{\text {th }} \text { specie producing the signal } \\
& \beta_{\mathrm{i}}=\text { backscattering factor for } \mathrm{i}^{\text {th }} \text { specie } \\
& \Phi_{\mathrm{i}}=\text { ionization cross section for } \mathrm{i}^{\text {th }} \text { specie } \\
& \Psi_{\mathrm{i}}=\text { transition probability for } \mathrm{i}^{\text {th }} \text { specie } \\
& \mathrm{R}=\text { surface roughness factor } \\
& \mathrm{T}_{\mathrm{i}}=\text { instrument transmission factor for the } i^{\text {th }} \text { specie } \\
& \lambda_{\mathrm{i}}=\text { mean free path of the } i^{\text {th }} \text { specie } \\
& \theta=\text { angle of incident beam from sample normal }
\end{aligned}
$$

This equation assumes for a bulk, uniformly distributed samples of element i.

The mean free path, $\lambda_{\mathrm{i}}$, determines the depth from which electron can escape and be captured by the analyzer. A detailed discussion of mean free path is presented in the next section. As an electron beam is used to probe the surface, the secondary electrons backscatters from the sample can also cause Auger electron emission (as opposed to that caused by primary probing electron beam), leading to a false enrichment of overall Auger signal intensity and the necessity of defining backscattering factor. The backscattering factor, $\beta_{i}$, depends mostly on the atoms and very little on the electron beam energy ${ }^{67}$. For these experiments, the thin film grown 
and the substrate were of very similar or the same materials and have the same atomic (diamond/zinc blend) structure. The backscattering ratio could be dropped from the above ratio calculation for this reason. The roughness factor, R, was ignored as a low-order influence.

For analysis on homoepitaxial growth on Si substrate, the C-KLL intensity is normalized to the peak-to-peak intensity of the pre-growth Si-LMM scan to eliminate effects of variation in day-to-day instrument operation. Similarly for the heteroepitaxial growth on $6 \mathrm{H}-\mathrm{SiC}$ substrates, the C-KLL peak-to-peak intensities were reference to a single pre-growth C-KLL scan. This ratio data analysis technique was utilized to eliminate the instrumental variables. The measurement was thus $\mathrm{I}_{\mathrm{i}} / \mathrm{I}_{\text {ref }}$ for the sample. For this purpose, quantities that were only dependent on the instrument operation and not the samples, such as $I_{p}, A, T_{i}$, and $\theta$, were common terms and not considered for this ratio. To standardize the results, all the AES scans were referenced back to a single pre-growth, Fenner-etched Si surface or a pre-growth C-KLL scan on $6 \mathrm{H}-\mathrm{SiC}$ surfaces. The ratios between individual scans and the reference scan were examined instead of the absolute intensity. Therefore, this ratio was represented as

$$
\frac{I_{i}}{I_{r e f}}=\frac{N_{i} \cdot \beta_{i} \cdot \Phi_{i} \cdot \Psi_{i} \cdot T_{i} \cdot \lambda_{i}}{N_{r e f} \cdot \beta_{r e f} \cdot \Phi_{r e f} \cdot \Psi_{r e f} \cdot T_{r e f} \cdot \lambda_{r e f}}
$$

The remaining terms were lumped together as an elemental sensitivity factor $S_{x}$. Tables and figures of $\mathrm{S}_{\mathrm{x}}$ as a function of atomic number, Auger transition, and incident electron energy are available in our lab handbook. ${ }^{68}$ The terms $\Phi_{\mathrm{i}}, \Psi_{\mathrm{i}}$, and $\mathrm{T}_{\mathrm{i}}$ can be determined more accurately individually. However, because of instrumental influences and sample-dependent matrix effects, experimental determination of $S_{x}$ for the materials of interest with the specific analyzer to be used lead to the highest accuracy. 
Using this simplification, the relative atomic percentage of elements with respect to each other was

$$
x=\frac{I_{i} / S_{x_{i}}}{I_{i} / S_{x_{j}}} .
$$

Normalized absolute intensities was determined relative to a known pure-component reference $\left(\mathrm{N}_{\text {ref }}=\right.$ known $)$ by

$$
I_{i}=I_{r e f} \cdot\left(N_{i} / N_{r e f}\right) \cdot\left(S_{x_{i}} / S_{x_{r e f}}\right) .
$$

Using an as grown high-stoichiometry and thick $\mathrm{SiC}$ film, the ratio of $\mathrm{C}$ to $\mathrm{Si}$ in $\mathrm{SiC}$ is determined to be 0.68. (Appendix B)

\subsubsection{Mean Free Path Estimations}

As previously stated, in Auger spectroscopy, the electron kinetic energy is used to characterize the chemical composition of the thin film as well as the chemical state of its atoms. When struck by high energy electrons, the atoms emit their own electrons traveling through matter before reaching the surface and being collected by an analyzer. Some electrons undergo collisions (or inelastic shocks) during their paths to the surface, and the average distance they travel between the inelastic shocks is the inelastic mean free path (IMFP), $\lambda$. The initial kinetic energy of electrons and the nature of the medium present have the greatest influence on the value of the mean free path.

As some electrons undergo one or multiple inelastic shocks, they also experience random changes of energy that contribute to the background noise. Signals of those electrons that "escape" the medium without any inelastic shocks form the peaks in an Auger spectrum. Almost all "escaped" electrons come from within the depth equivalent to 6 mean free paths (Figure 3- 


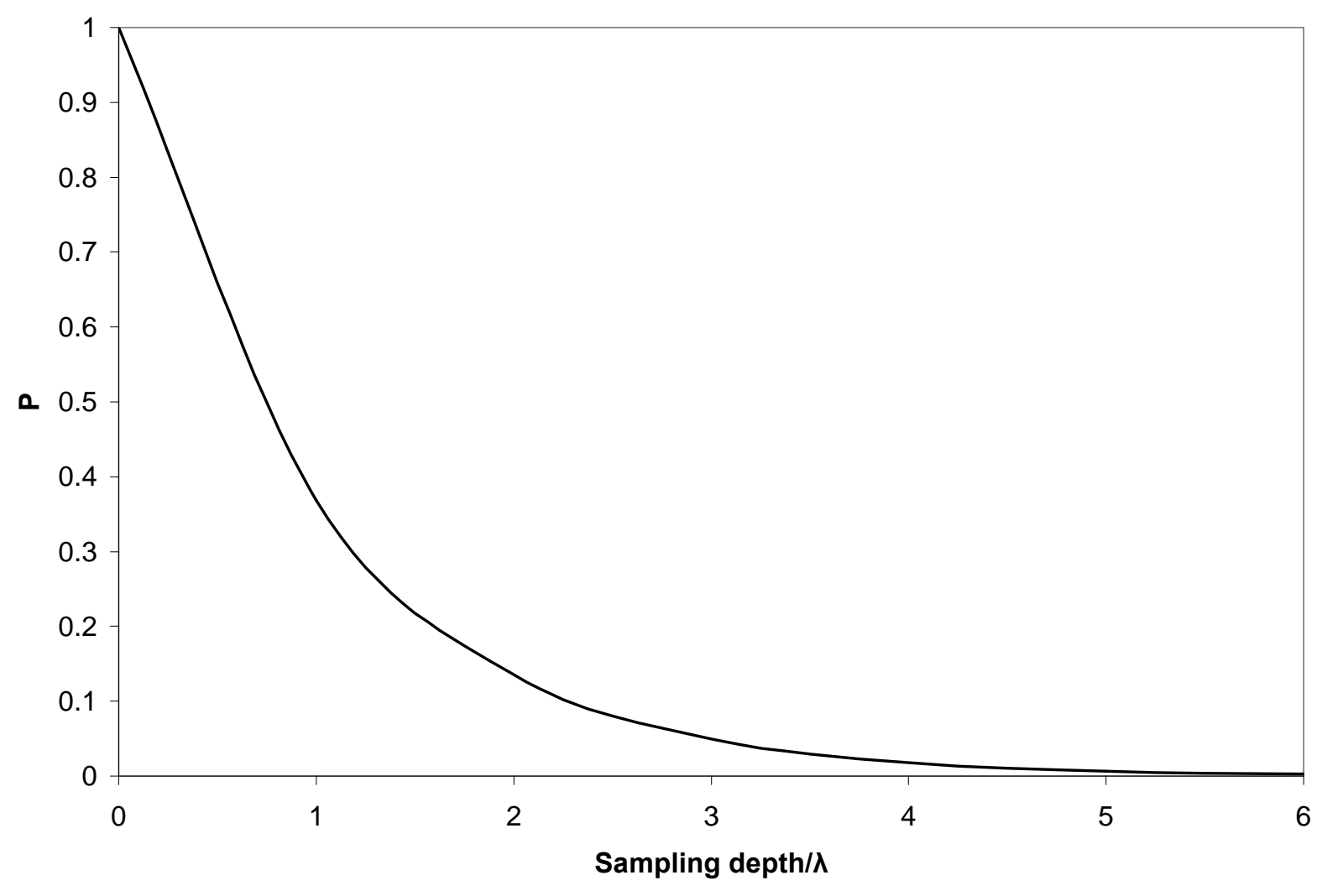

Figure 3-10. Probability of emitted electrons reaching the surface.

10), which means, for example, Auger has a sampling depth of around $3 \mathrm{~nm}$ for Si-LMM in silicon substrates, making AES an extremely surface sensitive technique.

The experimental results by Lindau and Spicer $^{69}$, and Powell ${ }^{70}$ have shown that the IMFP ( $\lambda$ ) has a minimum value for energies around $100 \mathrm{eV}$ and that at higher energies it varies "roughly as square root of electron energy". According to Seah and Dench's analysis of the inter-element and inter-compound effects ${ }^{71}, \lambda$ is related to the atoms size and the most accurate relation is $\lambda=2170 E^{-2}+0.72(a E)^{1 / 2}$ for inorganic compounds, where $a$ is the monolayer thickness (nm) and $E$ is the electron energy above the Fermi level in eV. A database developed by the National Institute of Standards and Technology based on many such experimental references is used to obtain necessary mean free paths for this study ${ }^{72}$. The Gries (G-1 equation) 
and Tanuma, Powell, and Penn (TPP-2M equation) predictive formulas in the database show the mean free path value of carbon in graphite to be 6.36 Angstroms, carbon in diamond 3.98 Angstroms, carbon in silicon carbide 9.22 Angstroms, and silicon 5.18 Angstroms.

\subsubsection{AES Layer Modeling}

Knowing the mean free path aids in calculating the probability of these emitted electrons actually reaching the surface. Relating to the sampling depth, $d$, this probability is determined by equation as follows: $P=e^{(-d / \lambda)}$. Figure 3-11 gives the probability of electrons coming through a depth of medium measured in multiples of mean free path. Under such a homogeneous condition, it is clear that few electrons can reach the surface from a depth equivalent to six times the mean free path. Consequently, the signal from these emitted electrons becomes gradually weaker as deeper layers of the thin films are probed. Since the actual signal intensity from each layer is directly related to the number of emitted electrons, it can be estimated using an equation by Yates and co-workers ${ }^{73}$

Top layer: $\quad I_{1}=I_{0}$

Second layer: $\quad \frac{I_{2}}{I_{0}}=e^{-(d / \lambda)}$;

Third Layer: $\quad \frac{I_{3}}{I_{0}}=e^{-(d / 2 \lambda)}$;

and the total intensity $I=I_{1}+I_{2}+I_{3}+\ldots \ldots$

The concept behind this model is that each monolayer of a pure element contains a known number of atoms that will produce the same measured AES peak intensity. The top layer will attenuate the measured AES signal from the second layer because some of the electrons that would have been detected will be scattered by the electrons in the top layer. Similarly, the 
subsequent layers attenuate more and more. The level of attenuation is determined by the film thickness above the layer, the mean free path of the element, and the number of atoms in the layer. Different combinations of monolayers or makeup within the layer attenuate the signal more or less.

In real thin film growth, homogeneity is not a common occurrence from layer to layer, or even within the same layer. The assumption of layer upon layer of homogeneous SiC film grown on top of the Si substrate is far from accurate. It is difficult to predict the actual layer-tolayer structure because the AES signals vary among different forms of carbon. For a given number of carbon atoms in a compound sampling depth, the measured carbon intensity will be different if the carbon atoms are uniformly distributed in the sampling depth than if they are in or under layers of different densities.

AES scan line shapes of Si-LMM and C-KLL suggested thin films in this study very likely consists of carbon in all three forms $\left(\mathrm{SiC}, \mathrm{sp}^{2}-\mathrm{C}\right.$, and $\left.\mathrm{sp}^{3}-\mathrm{C}\right)$. The model is thus the summation of the continuous intensity. Implemented as an Excel spreadsheet, with the intensity input of one monolayer of carbon in $\mathrm{sp}^{3}, \mathrm{sp}^{2}$, and $\mathrm{SiC}$ relative to pure silicon reference, the overall saturated intensity is predicted for various film-layer combinations as well as the effect of a silicon or $\mathrm{sp}^{3}-\mathrm{C}$ over-layer on $\mathrm{SiC}-\mathrm{C}$ intensity. The information, including atomic densities, relative sensitivity factors, lattice constants, mean free paths, plane spacing, etc., contains significant errors, and therefore this model is only to help understand how carbon intensities could be changing under different layer formations, not to prove a particular layer-by-layer configuration. 


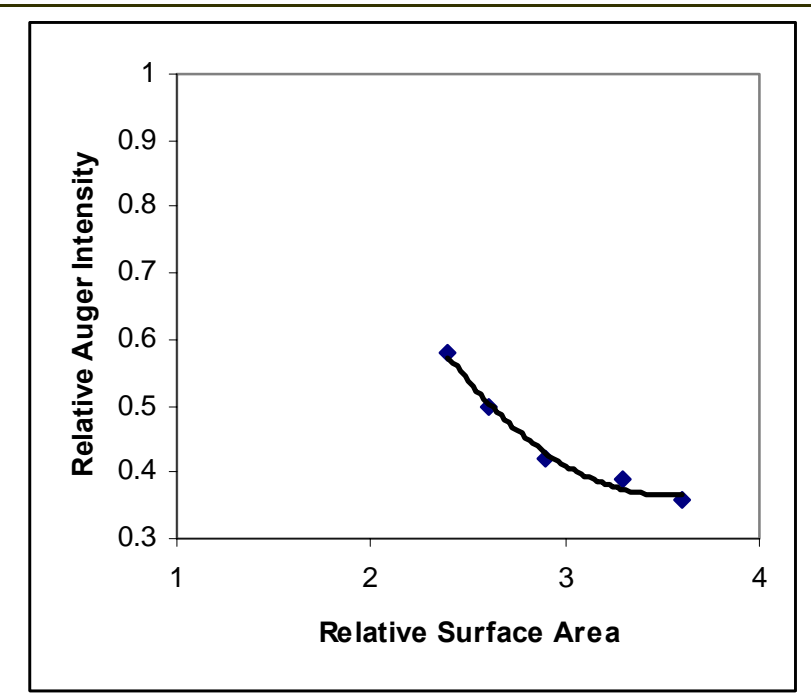

Figure 3-11. Auger intensity and surface roughness study. (Plot based on data extracted from $\mathrm{Wu}$ and Butler.)

\subsubsection{Roughness Effect on AES}

The AFM examinations of individual samples and previous studies by Ziemer showed the roughness to be in the range of 3 to $15 \AA$. It is well known that surface roughness reduces Auger signal intensity due to added electron scattering, therefore leading to doubt in the accuracy of the quantitative analysis. $^{74,75,76}$

The first known quantitative analysis

of the relationship between Auger intensity and surface area roughness data was done by Wu and Butler. ${ }^{77}$ Auger peak-to-peak heights were recorded for surfaces with different Relative Surface Areas" (actual surface area/reference surface area) ranging from 2.4 to 3.6. Most of their samples had cone-shaped roughness features 5 microns in height and diameter, which were thousands to tens of thousand times larger than those in present work. These suffered Auger intensity loss from $42 \%$ to $64 \%$. (Figure 3-11) If such a relationship still applies to our Fenner etched samples, with nanometer-scaled roughness and Relative Surface Areas slightly larger than 1, the trend line based on their study would indicate that there should be little to no intensity loss for such cases.

It is noted as well that the authors used their "relatively flat sample", with a relative surface area of 1.1, as the Auger intensity reference, i.e., they knew or speculated that such a sample would suffer no intensity loss compared to a perfectly flat surface. 
So far, no literature has been located to verify quantitatively the effect surface roughness has on Auger intensity on the orders of nanometers or angstroms, i.e., experimental parameters similar to ours, and our data interpretation and analysis will be open to possible errors due to such an effect.

\subsubsection{AES Error Analysis}

Previous researches in this laboratory by Ziemer has determined the error in the AES intensity measurement to be $\pm 3.6 \%$ and the error in normalized intensity to be $\pm 5.1 \%$. Error bars are displayed on all charts to reflect such when applicable.

\subsubsection{Reflection High Energy Electron Diffraction (RHEED)}

RHEED is widely used as an in-situ surface-sensitive crystal structure characterization technique for the thin films during the growth process. It was used to monitor the growth progress and in-plane atomic spacing.

In RHEED, a well collimated, monoenergetic high-energy electron beam, about $0.1 \mathrm{~mm}$ diameter, between 5 and $100 \mathrm{keV}$, strikes the surface at low grazing incident angles $\left(0.1\right.$ to $5^{\circ}$, depending on electron energy $)^{78}$. Because of the grazing angle, momentum of the electrons normal to the surface is very small. This combined with the strong interaction between beam electrons and the sample, result in a beam penetration depth of only a few $\AA$. Consequently, the technique is very surface sensitive much like Auger Electron Spectroscopy. As the sample is rotated about an axis normal to its surface, diffraction patterns related to the different surface crystallographic directions can be obtained to establish the surface periodicity and symmetry of the surface. This technique provides a qualitative picture of the crystal surface, and is especially useful in the early stages of crystal growth. 


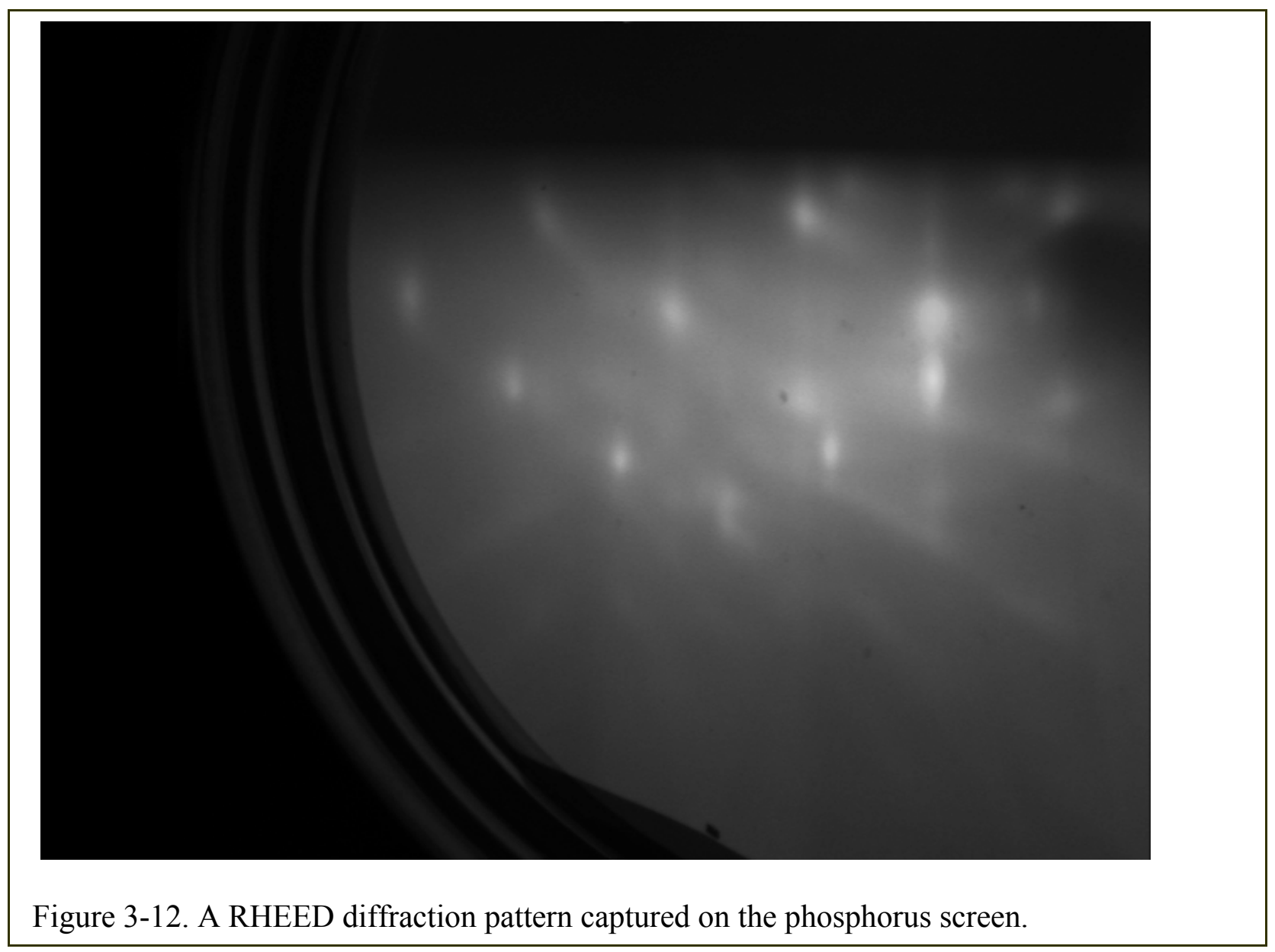

The scattered electrons (diffracted by the lattice) impinge onto a phosphorus screen and the resulting image (Figure 3-12) was recorded in the form of photos using a charge-coupled device $(\mathrm{CCD})$ camera.

The schematic of the RHEED setup in our laboratory is shown in Figure 3-13. The RHEED system used in these experiments was a Staib Instrumente Model RH 15. The beam energy of the electron gun was controlled in the range of $10-15 \mathrm{keV}$, and beam current 1.0 $1.5 \mathrm{~A}$.

The substrate acted as a two-dimensional grating, which diffracts the electron beam in the form of a series of streaks and/or spots, as shown in Figure 3-12. The beam path is orthogonal to the flux beams associated with MBE growth. 


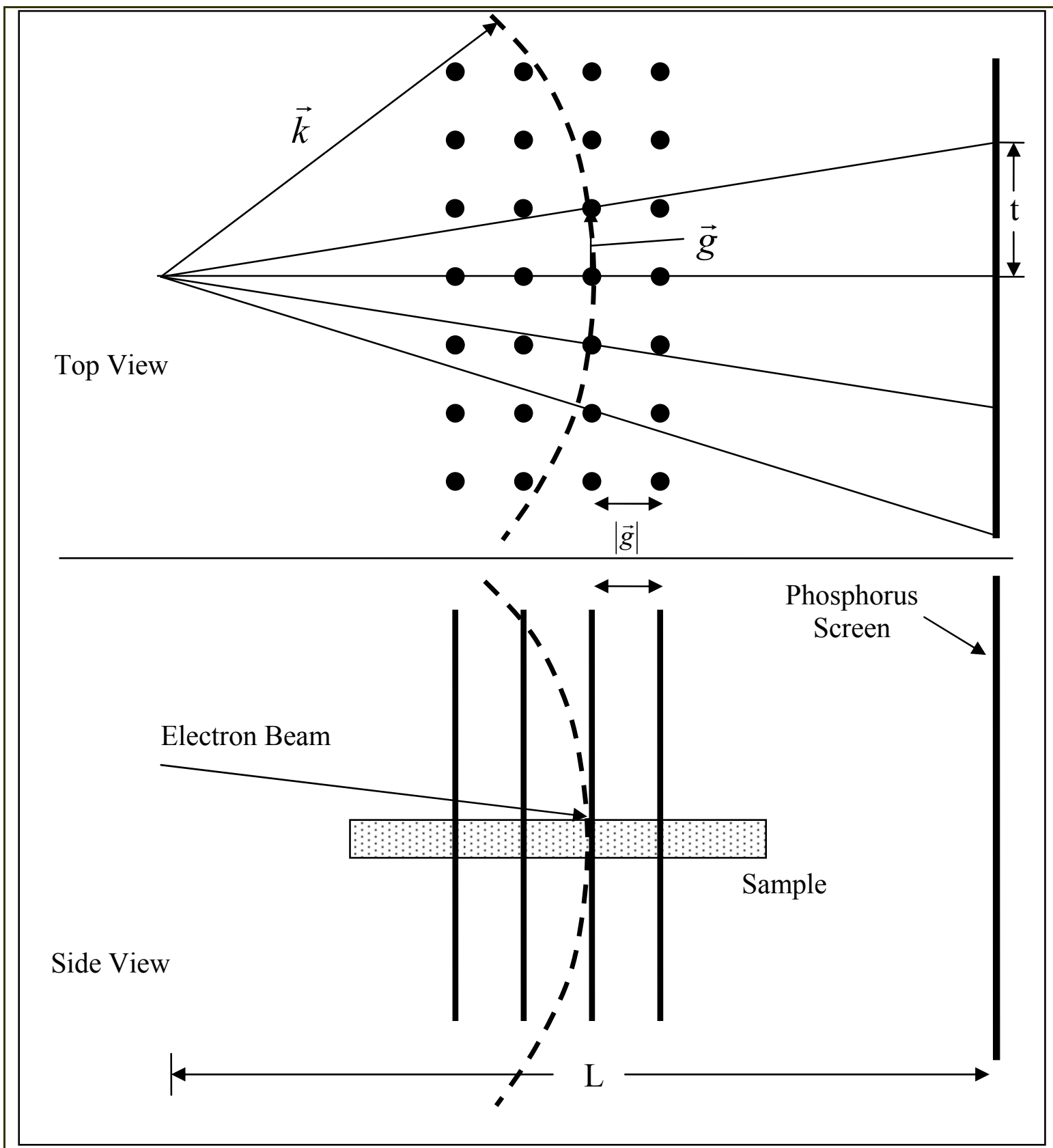

Figure 3-13. The Braggs Diffraction condition in RHEED. Concept of drawing taken from http://www.stoner.leeds.ac.uk/techniques/rheed.htm

The de Broglie wavelength, $\lambda$, of the incident beam electrons is given by

$$
\lambda=\sqrt{\frac{150.4}{E}} .
$$


where $\mathrm{E}$ is the electron energy in $\mathrm{eV}$. For electrons in the range of $10-15 \mathrm{keV}$, wavelength varies from 0.1 to $0.12 \AA$. The magnitude of the corresponding wave vector, $\vec{k}$, is given by

$$
|k|=\frac{2 \pi}{\lambda} .
$$

In Figure $3-13^{79}$, the Braggs diffraction conditions are met whenever the Ewald Sphere intersects the sample surface's reciprocal lattice rods, which are normal to the sample plane. With that understanding in mind, it is obvious that the reciprocal lattice of a 2-D surface is a lattice of rods, a "bed of nails" structure called Bragg rods. Mathematically, this is given by the condition $\vec{k}^{\prime}=\vec{g}+\vec{k}$, where $\vec{k}$ is the incident electron wave vector, and $\vec{k}^{\prime}$ is the diffracting one, and $\vec{g}$ is a reciprocal lattice vector. (Figure 3-14) Since elastic electron scattering is assumed here, $|\vec{k}|=|\vec{k}|$. The Ewald Sphere has its origin at the origin of $\vec{k}$, and a radius of $|\vec{k}|$, or it can be understood as an infinite number of $\vec{k}^{\prime}$ vectors pointing to all directions in space. Hence diffraction occurs for all the vectors that intersect the reciprocal lattice rods.

RHEED diffraction is represented by the intersection of these rods and the Ewald sphere, and theoretically, for a perfect surface, would be spots lying on an arc (the Laue Circle). In practice, the spots tend to be streaked to a certain extent due to a number of facts causing broadening of the rods: the energy and angular spread of the incident electron beam, surface roughness and defects, (inelastic) phonon scattering, and refraction at the surface. ${ }^{80}$ RHEED patterns will also show streaking from a flat surface if the in-plane ordering length is less than the electron mean free path. ${ }^{81}$

The magnitude of $\vec{g}$ is 


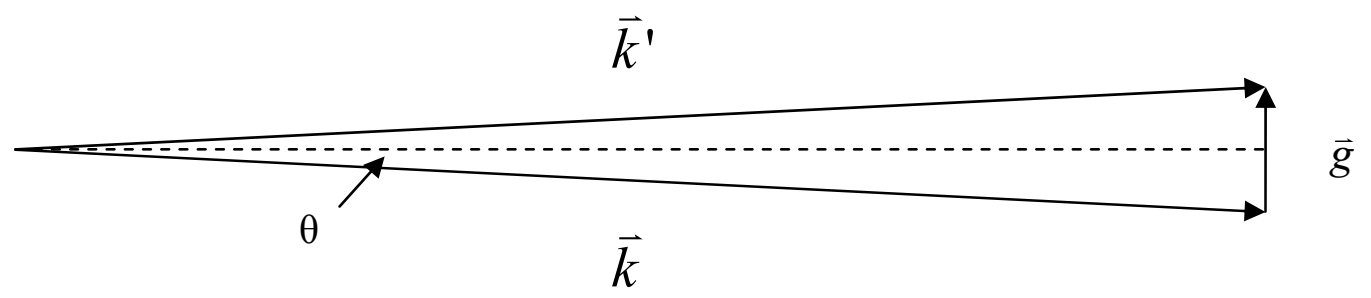

Figure 3-14. Bragg Diffraction Condition Geometry.

$$
|\vec{g}|=\sqrt{h^{2}+l^{2}} \frac{2 \pi}{a}
$$

where $\mathrm{h}$ and $\mathrm{l}$ are the indices of planes, e.g. (00), (01), with intersecting reciprocal lattice rods. From the geometry of the Ewald sphere, the Bragg condition is met when

$$
\frac{|\vec{g}| / 2}{|\vec{k}|}=\tan \theta,
$$

as seen in Figure 3-14. Moreover, referring to he sample-screen geometry in Figure 3-14

$$
\frac{t}{L}=\tan 2 \theta
$$

Combining Equations 3.18 and 3.19, and because the angle $\theta$ is so small, $\tan 2 \theta \approx \sin 2 \theta \approx 2 \sin \theta \approx 2 \tan \theta$. Thus

$$
\frac{|\vec{g}|}{|\vec{k}|}=\frac{t}{L}
$$

Substituting into Equations 3.16 and 3.17,

$$
\frac{\sqrt{h^{2}+l^{2}} \lambda}{a}=\frac{t}{L}
$$


Understandably, for each unique surface structure, there is a corresponding diffraction pattern that is the fundamental reflection for that surface structure. Using the information from diffraction pattern, the actual surface lattice parameters can be determined. ${ }^{82}$ As the radius of the Ewald sphere is about two orders of magnitude greater than the modulus of the reciprocal lattice vector, the angle between points intersecting the sphere, $2 \theta$, will remain almost the same. In 2D, this means the Ewald sphere will touch only a few rods on either side of the (00) rod. Suppose the Ewald sphere intersects two adjacent reciprocal lattice rods spaced apart by a distance $\mathrm{b}$, i.e., $b=\frac{2 \pi}{d_{\text {parallel }}}$, then

$$
\begin{aligned}
& \tan 2 \theta=\frac{b}{\left|\vec{k}_{0}\right|}=\frac{t}{L} \\
& d_{\text {parallel }}=\frac{2 \pi L}{\left|\vec{k}_{0}\right| t}=\frac{L \lambda_{0}}{t}
\end{aligned}
$$

where $d_{\text {parallel }}$ is the actual distance between rows of atoms corresponding to the reciprocal lattice rods and $t$ is the distance between streaks in the diffraction pattern. Using a well-known material and its lattice parameter, the product $L \lambda_{0}$, which remains constant, can be found. Therefore, RHEED can be used to determine the crystal structure by calculating $d_{\text {parallel }}$ in different directions.

This as well as the examination of sample surface roughness/disorder previously mentioned would be the main purpose of using RHEED in this research.

\subsubsection{Field Emission Scanning Electron Microscope (FESEM)}

Unlike a conventional microscopy, which uses a series of lenses to direct light waves and create magnified images, the FESEM does so by using electrons and is able to produce much 
higher magnifications. A high-voltage, heated filament (usually between $1 \mathrm{kV}$ and $20 \mathrm{kV}$ ) emits a beam of electrons, which are directed and focused by a series of magnetic lenses to a fine, microscopic size spot. A set of scanning coils then "sweep" the beam across the sample while dwelling on points for a pre-determined length of time (usually in microseconds). The electron beam is small enough to generate very high-resolution images of nanometer scale. As the electrons strike the sample surface, the low-energy secondary electrons are ejected as a result of inelastic collisions. The principle of FESEM is that the topography strongly influences the emission of secondary electrons, thus, non-flat surfaces appears brighter on the image screen than flat surfaces. This allows the examination of sample's cross-section, the construction and growth details, and possibly evaluation of film thickness.

The microscope is located inside a vacuum column to prevent electron beam scattering and gas molecule ionization or reaction with the filament, thus ensuring proper operation and accurate data collection.

Samples were mounted with conducting double-sided graphite tape in order to further enhance conduction of electrons away from the sample to prevent charging.

Some of the FESEM photos were taken at Pennsylvania State University's Nanofabrication facility with the help of Dr. Ed Basgall using a LEO model 1530. Photos of between $100,000 \times$ and $400,000 \times$ magnification were taken at $20 \mathrm{kV}$ operating voltage $(1.2 \mathrm{~nm}$ point-to-point resolution) through a solid state backscattered in-lens detector.

The majority of the FESEM photos were taken at WVU using a Hitachi HighTechnologies S-4700 model at between 1 to $5 \mathrm{kV}$ at 4-12 mm working distances. 


\subsubsection{Atomic Force Microscopy}

Atomic force microscopy (AFM) is a type of scanning force microscopy, where a sharp probe is scanned across the surface and the probe-surface interaction is recorded. It is a relatively new surface technique invented in $1986 .{ }^{83}$ Unlikely the better known Scanning Tunneling Microscopy, it is capable of imaging electrically non-conducting surface as well. (Concept of this writing is partially taken from Veeco Instrument, Inc. AFM operational manual)

An atomic force microscope uses an atomically sharp tip placed sufficiently close to the surface of the sample. The tip, acting as a cantilever, exerts a force on the sample, and the deflections (as small as $10^{-4} \AA$ ) of the tip with its force-sensing edge are recorded. The force is calculated from Hooke's Law: $F=-k x$. Here $\mathrm{x}$ is the cantilever deflection, and the mechanical properties of AFM are based the atomic equivalent spring constant,

$$
k=\varpi^{2} m \sim 10 N / m,
$$

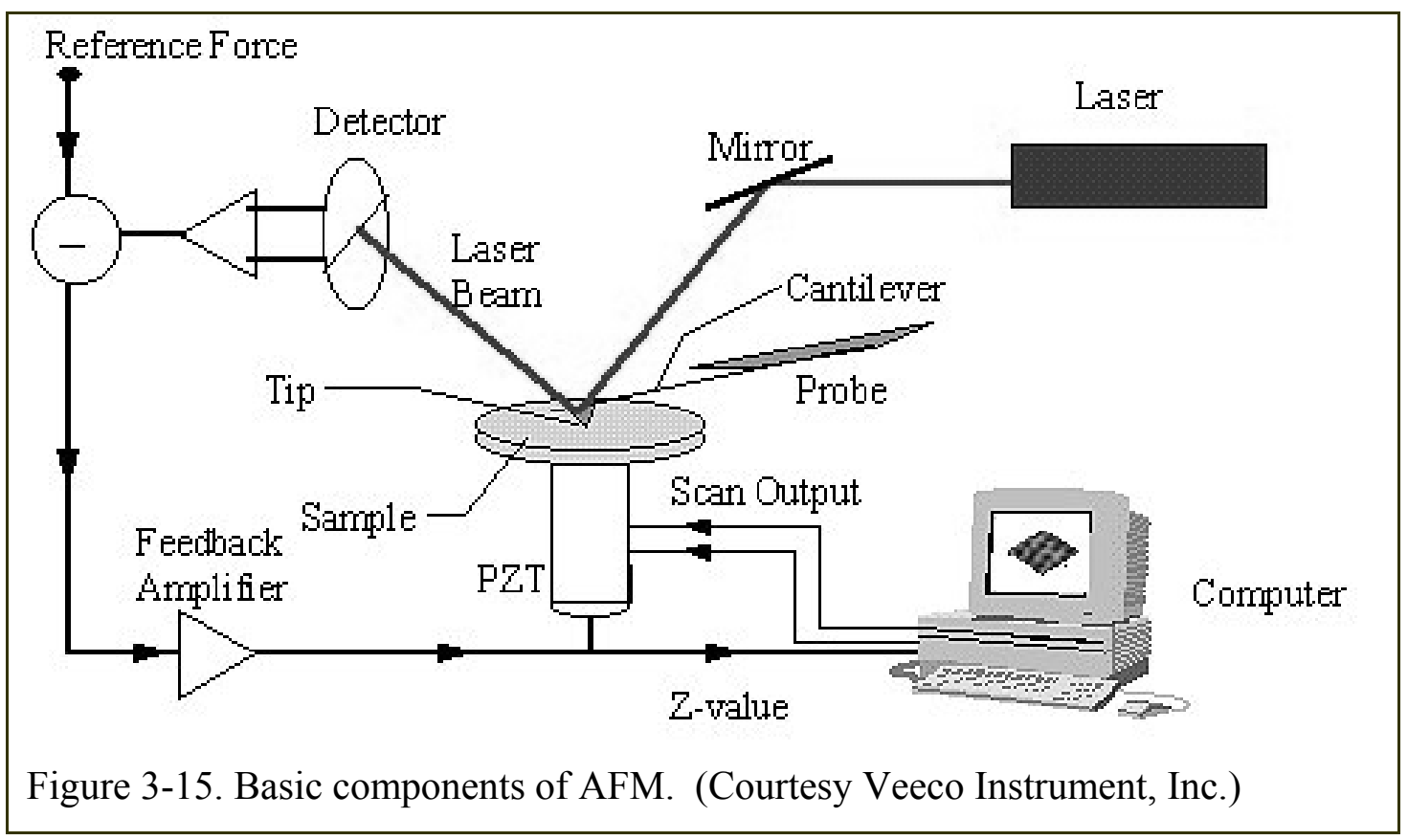




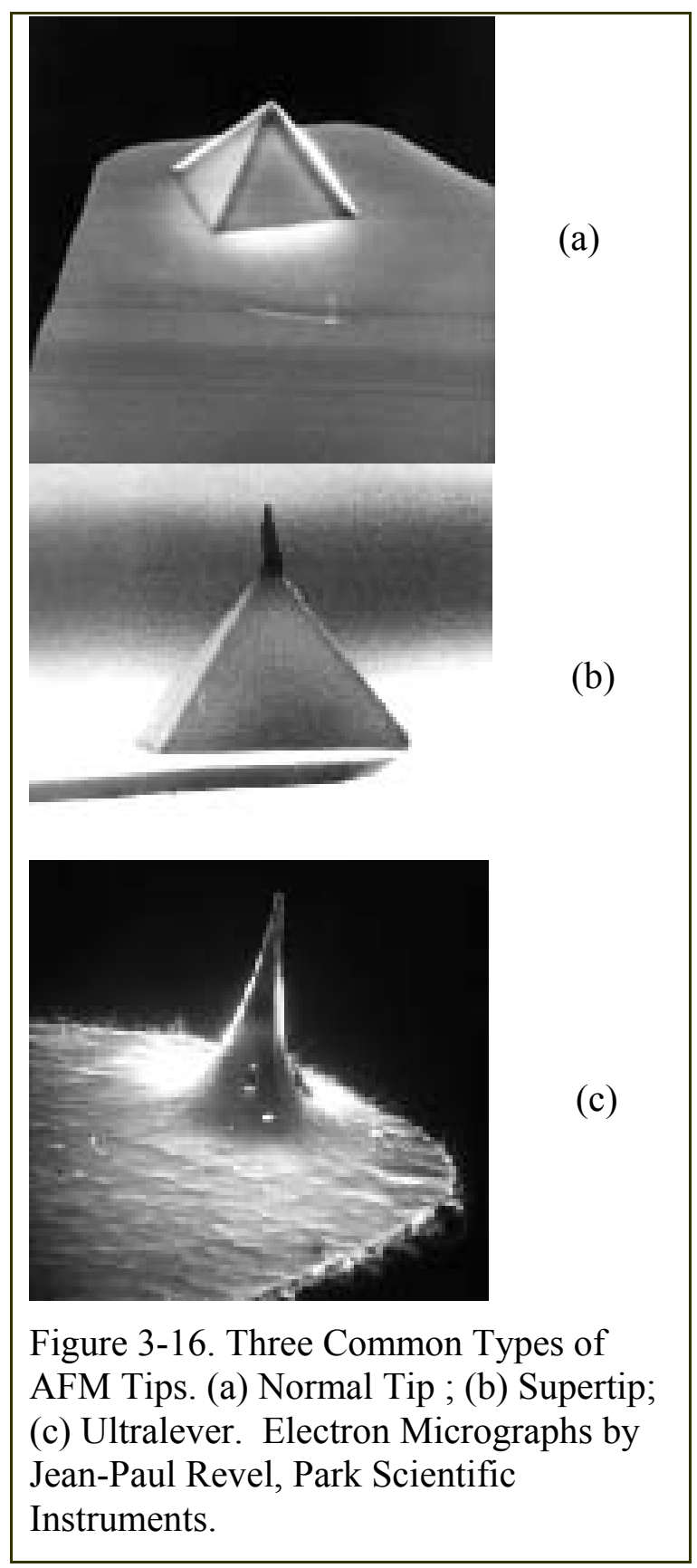

where the mass of an atom $\mathrm{m} \sim 10^{-25} \mathrm{~kg}$ and the frequency of vibration of a typical atom is $\omega(\sim$ $\left.10^{13} \mathrm{rad} \cdot \mathrm{s}^{-1}\right)$. The spring constant of an AFM cantilever usually does not exceed $10 \mathrm{~N} / \mathrm{m}$ to avoid damages to sample's surface. AFM cantilevers are purchased with their attached tips from commercial vendors, who manufacture the tips with a variety of microlithographic techniques. AFM tips are actually rounded off and the "end radius" is used to evaluate and categorize the tips. As shown in figures below, the "normal tip" is a $3-\mu \mathrm{m}$ tall pyramid with a 30-nm end radius; the "super tip" has an elongated head and sometimes smaller end radius designed for probing pits and crevices; and the "ultralever" offers a sub $10-\mathrm{nm}$ end radius. (Figure 3-18) Normal tips made of $\mathrm{Si}_{3} \mathrm{~N}_{4}$ were used for the AFM scans in these studies.

As the tip scans the surface of the sample, moving along the contour of the surface, the laser beam is deflected off the attached cantilever into a photodiode. The difference in light intensities between the upper and lower photodetectors is measured and then converted to voltage. Feedback from the photodiode difference signal, through software control from the 
computer, which enables the tip to maintain either a constant force on the sample (contacting mode AFM) or constant height above the sample (non-contact mode AFM).

In the contact mode, the cantilever is pushed against the sample surface while a piezoelectric positioning element setting the deflection force. The deflection of the cantilever is sensed and compared in a DC feedback amplifier to the desired value of deflection. If the measured deflection is different from the desired value the feedback amplifier applies a voltage to the piezoelectric scanning tube (PZT) to raise or lower the sample relative to the cantilever to restore the desired value of deflection. The voltage that the feedback amplifier applies to the piezo is a measure of the height of the feature on the sample surface. The main problem with the contact mode is the excessive tracking forces applied by the probe to the sample, causing damage to both the tip and the sample surface. In addition, semiconductors and insulators in this study can trap electrostatic charge, contributing to additional substantial attractive forces between the probe and the sample. These problems can be limited by minimizing the tracking force of the probe on the sample but not eliminated.

Such difficulties are eliminated in non-contact mode, where the scanning tip oscillates close to the sample surface. Attractive Van der Waals forces acting between the tip and the sample are detected, and topographic images are constructed by scanning the tip above the surface. As the attractive forces from the sample are substantially weaker, the tip must be given a small oscillation to detect the small forces between the tip and the sample by measuring the change in amplitude, phase, or frequency of the oscillating cantilever in response to force gradients from the sample. As such, non-contact imaging generally provides lower resolution and can also be hampered by the contaminant layer which can interfere with oscillation. This is a technique that is most effective in a vacuum environment. 
The tapping mode overcomes problems associated with friction, adhesion, electrostatic forces by alternately placing the tip in contact with the surface to provide high resolution and then lifting the tip off the surface to avoid dragging the tip across the surface. Tapping mode imaging is implemented in ambient air by oscillating the cantilever assembly at or near the cantilever's resonant frequency using a piezoelectric crystal at a frequency of 50,000 to $500,000 \mathrm{~Hz}$. As the oscillating cantilever begins to intermittently contact the surface, the cantilever oscillation is necessarily reduced due to energy loss caused by the tip contacting the surface. The reduction in oscillation amplitude is used to identify and measure surface features. When the tip passes over a bump in the surface, the cantilever has less room to oscillate and the amplitude of oscillation decreases. Conversely, when the tip passes over a depression, the cantilever has more room to oscillate and the amplitude increases (approaching the maximum free air amplitude). Unlike contact and non-contact modes, when the tip contacts the surface, it has sufficient oscillation amplitude to overcome the tip-sample adhesion forces. Also, the surface material is not pulled sideways by shear forces since the applied force is always vertical.

A three-dimensional image can finally be constructed by recording the cantilever motion in the $\mathrm{Z}$ direction or oscillation change as a function of the sample's $\mathrm{X}$ and $\mathrm{Y}$ position to obtain atomically resolved images. The images taken generally require further processing before surface data can be retrieved. Due to the slight pendulum scanning motion, the topographic images tend to attain a slight curvature at the corners giving the images an unequal color mapping, also known as cross-coupling. This can be corrected using a "Flatten" option in the image processing software. The "Filter" and "Deglitch" options can remove signals caused by mechanical vibrations, current surge, and other stray noises that are superimposed onto the AFM 
image during scanning. The surface features of interests for this research consist of maximum peak-to-valley, average roughness, and median and mean height values.

AFM images were collected at two different locations: a contact mode only Model 3A by Digital Instrument located in Dr. Myers' laboratory in the Department of Physics at WVU; and a JEOL Model JSPM-4210 located National Energy Technology Laboratory in Morgantown, West Virginia. The latter model is capable of surveying in both tapping mode and contact mode, and the majority of the AFM images were collected using the tapping mode. AFM data were extracted and processed using WinSPM System software provided by JOEL.

\subsubsection{Measured Surface Roughness}

Surface studies as characterized by AFM consists of three main parameters: roughness, step size, and step height, with the latter two applicable only to the hydrogen etched $6 \mathrm{H}-\mathrm{SiC}$ samples. The most often used roughness parameters are the average roughness, $R_{a}$, and the rootmean-square roughness $R_{r m s}$. The average roughness is defined as the sum of the areas above and below the mean surface line, divided by the length of the measurement line; and the rootmean-square roughness is defined as the value obtained by the root-mean square calculation of the surface profile values. ${ }^{84}$ It can be expressed as:

$$
R_{r m s}=\sqrt{\frac{1}{l} \int_{0}^{l} y^{2}(x) d x}
$$

Here $y$ is the dimension perpendicular to the surface, and $l$ is the measurement length, in the direction of the surface, defined by $x$. Root-mean-square roughness is a commonly used measurement unit in AFM surveys. The average values of the $\mathrm{R}_{\mathrm{rms}}$ for both Fenner-etched $\mathrm{Si}$ and hydrogen-etched $\mathrm{SiC}$ substrates (as measured on flat terraces) were on the magnitude of 1 to 3 angstroms. 


\subsection{Experimental Procedures}

\subsubsection{Si Substrates and Substrates Preparation}

The Si substrates used in this study were $10 \mathrm{~mm} \times 24 \mathrm{~mm}$ rectangular samples cleaved from 4-inch diameter $\operatorname{Si}(100)$ wafers doped n-type. It was cut so that the [110] plane is always on the edge of the longer side. (Figure 3-19) The samples were mounted to the sample holder with molten indium-tin alloy (melting point $=117^{\circ} \mathrm{C}$ ) heated to $150^{\circ} \mathrm{C}$, and they were fastened on the edge by tantalum wire. However, this method was less than desirable because the area of effective thermal contact gradually decreased as indium evaporates at the growth temperature. Colloidal graphite was used later to replace the alloy as the heat conducting media.

Due to the native silicon oxide formation and carbon contamination, the substrate surface required cleaning prior to growth procedures. The two primary cleaning methods used in this laboratory were the in-situ argon-ion etch and the ex-situ Fenner etch. In general, the ex-situ cleaning process removed the contaminants and left either a hydrogen-terminated surface or protective oxide-covered surface that is stable for transport to the process system. Fenner etch was a hydrofluoric acid-based wet chemical process developed by D. B. Fenner ${ }^{85}$ and later

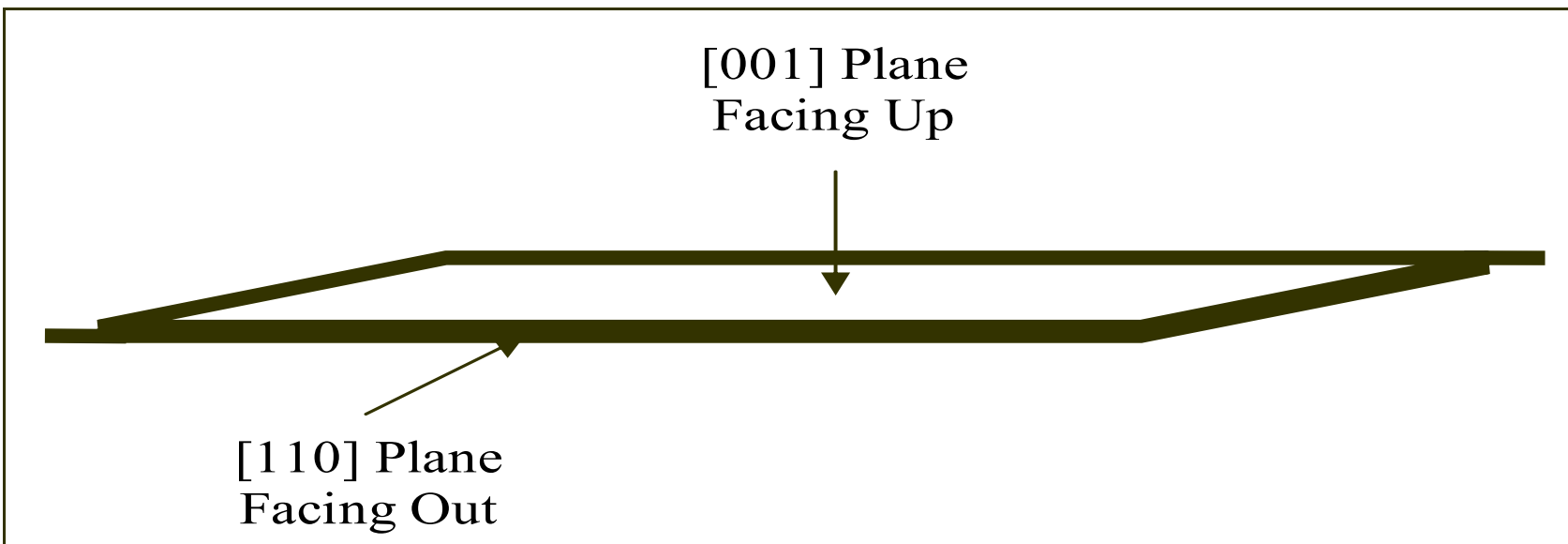

Figure 3-17. Cleaved sample substrate. 
modified by Lauren Hirsch ${ }^{86}$ of the WVU Physics Department. This procedure yielded a hydrogen-terminated $1 \times 1 \mathrm{Si}(100)$ surface. This ex-situ method had the sample immersed, rinsed, and dipped in different chemical solutions to remove the carbon contaminants and oxide residue. To ensure the lowest level of impurity, all chemicals used were electronic or tracemetal grade. In addition, distilled and de-ionized (DI) water $(>18.0$ megaohm-cm) was used to rinse the sample and high purity nitrogen is used to blow-dry the sample when necessary. Prior to the Fenner etch, the sample was de-greased sequentially in three solvents (acetone, trichloroethylene, and methanol) in order to dissolve all organic contaminations. After a DI water rinse, the sample was submerged in a heated $\left(80^{\circ} \mathrm{C}\right)$ solution of $\mathrm{H}_{2} \mathrm{O}: \mathrm{NH}_{4} \mathrm{OH}: \mathrm{H}_{2} \mathrm{O}_{2}$ (5:1:1) to form a basic thin oxide layer which was subsequently removed by a dip into $1 \% \mathrm{HF}$ solution for 15 seconds. After another DI water rinse, the substrate was then placed into a solution of $\mathrm{H}_{2} \mathrm{O}: \mathrm{HCl}: \mathrm{H}_{2} \mathrm{O}_{2}(6: 1: 1)$ to form an acidic thin oxide layer. Finally a solution of $\mathrm{HF}$ : $\mathrm{H}_{2} \mathrm{O}$ : reagent alcohol $(1: 1: 10)$ was used to removed both the thin oxide layer formed with contaminants and the native silicon oxide, forming a fresh hydrogen-terminated $1 \times 1$ Si substrate surface.

Ziemer's research indicated that the ex-situ Fenner etch also produces a smoother substrate surface $\left(\mathrm{R}_{\mathrm{rms}}=2.5 \AA\right)$ when compared to the in-situ argon etch and annealed (AEA) method $\left(\mathrm{R}_{\mathrm{rms}}=4.2 \AA\right){ }^{48}$ Her research also revealed that initial reactions on the hydrogenterminated surface produced a more effective diffusion barrier against the unwanted activities from the Si substrate. Based on these findings, the current studies will utilize only the Fenner etch as the sample preparation method for heteroepitaxial film growth. 
Upon the conclusion of the Fenner etch, the substrate was placed onto the sample holder, which has a colloidal graphite pasted on the surface. This colloidal graphite, when heated, acted as a radiative heat source as well as a heat-transfer media between the sample and its holder.

\subsubsection{UHV Chamber Heteroepitaxial Growth Studies on Si Substrate}

Typical growth run started with a substrate cleaned using Fenner etch. Once the sample was secured inside the system and the pressure reduced to the desired UHV level, the pre-growth surface chemical composition and crystal structure examination were performed using AES and RHEED. Methylsilanes were introduced into the chamber through a leak valve to a predetermined pressure calculated from the desired corresponding flux level $(0.342 \mathrm{ML} / \mathrm{sec})$. In the presence of the source, the substrate was heated to the growth temperature $\left(700^{\circ} \mathrm{C} / 800^{\circ} \mathrm{C}\right)$ quickly. This process took approximately 7 minutes, and that time period was included as part of total growth time. This was a critical requirement because Ziemer's research showed that an annealed Fenner etch surface was ultimately reconstructed into a $2 \times 1$ structure, which is unfavorable for $\beta$-SiC thin film growth. To minimize the effect of surface reconstruction, the substrate was thus heated in the presence of the gas source. The growth runs consisted of two different fluence levels that were calculated based on growth time periods of 15 minutes and 60 minutes $($ Flux $\times$ Growth Time $=$ Fluence $)$. During the growth period, in-situ analysis of the sample was performed using RHEED. The pressure was maintained constant until the end of the growth run when the gas flow was shut off before the heater was turned off. The cooling process generally lasted one hour before the sample was at an acceptable temperature for both transporting the sample and the AES analysis of film and substrate. This concluded the in-situ portion of a run. The sample was then removed from the system for ex-situ analysis such as 
FESEM (film/substrate interface structure and morphology) and AFM (surface morphology and roughness).

\subsubsection{H-SiC Substrates and Substrates Preparation}

In the other phase of the study, epitaxial thin films were grown on $6 \mathrm{H}-\mathrm{SiC}$ substrates to produce a smooth surface. Since commercially available SiC wafers were often covered by surface and subsurface defects as a result of the polishing process, they need to be treated before the growth process. One of the objectives of this research was to develop a method to consistently produce an ordered surface without such defects by using a high temperature hydrogen-etching furnace. Prior to high temperature hydrogen etching, all $\mathrm{SiC}$ samples were prepared using a standardized cleaning procedure. Studies done here had shown that debris and micro-particles, when left on the surface, could sometimes act as catalytic sites that accentuate or create defects.

The chemicals used in this procedure were in ultra high purity, electronic grade, or trace metal grade, and the water was distilled and de-ionized. The sample was first soaked in acetone for half an hour to dissolve any remaining organics. Then, to remove any solid residue, the surface was polished on a Politex Supreme buffing pad soaked in soapy water. This was followed by sequential acetone and methanol rinses. After blow-drying using ultra-high-purity nitrogen, a Q-tip soaked in acetic acid was used to wipe the surface systematically in horizontal, vertical, diagonal, and circular motions. This step was critical for removing most of the particles remaining on the surface. The substrate was again rinsed in methanol and blow-dried with ultra high purity nitrogen, and thoroughly rinsed in DI-water. It was then submerged in a 1:3 (HF acid:Reagent alcohol) solution for ten minutes to remove native oxides. Finally, the sample is 
blow-dried, examined under an optical microscope for un-wanted particles and water spots, and weighed using a micro-balance to determine its mass.

\subsubsection{High Temperature Hydrogen Etch of SiC}

The SiC sample prepared using the above method was immediately loaded into the high temperature hydrogen etch furnace. The hydrogen etch furnace is described in Section 3.3 in detail. Once both adapters are attached to the ends of the tube and properly sealed, the three way valve was directed to the down position, which allowed the entire tube to be pumped down to vacuum level of $10^{-2}$ Torrs (by MKS Baratron). This step served two purposes in both removing the air within the tube to prevent possible contamination associated with it and checking the level of seal/leakage achieved by the adapter. If the pressure was higher than desired, the adapters were taken off and reinstalled until a satisfactory level of seal was reached. The tube was then back-filled with Ar gas to a pressure of 15 psig. A Gastronic gas detector, sensitive to both $\mathrm{H}_{2}$ and Ar, was used to check for potential positive pressure gas leaks. Prior to starting the heating program, the two igniters as well as supply gas lines' pressure were tested. The heating program begins and ends with 15-minute Ar purge to flush out air at the start and to flush out $\mathrm{H}_{2}$ at the conclusion of the run. During the heating cycle, a stream of cooling water flowing at $1 \mathrm{~L} /$ min was maintained to keep the adapters at their proper operating temperature range to prevent degradation of the viton o-ring seal. In a typical run, the sample was ramped up to the etch temperature at a pre-determined rate $\left(400^{\circ} \mathrm{C} / \mathrm{hr}\right.$ up to $1000^{\circ} \mathrm{C}$ and $300^{\circ} \mathrm{C} / \mathrm{hr}$ thereafter), and maintained at the etching temperature $\left(T_{E}\right)$ for a specified dwell time $\left(t_{D}\right)$, which is typically between 0 and 4 hours. Upon the end of the dwell, the temperature was ramped down at the same rates. The ramp rates were set by the safety considerations based on the thermal expansion characteristics of the alumina reactor tube. During the etching process, gas flow rate $(\mathrm{F})$ and 
composition $\left(\chi_{\mathrm{H}_{2}}\right)$ are manually controlled via two rotameters for $\mathrm{H}_{2}$ and Ar respectively. At the end of the etching process, the sample was extracted, and had its mass recorded.

\subsubsection{UHV Chamber Homoepitaxial Growth Studies on 6H-SiC Substrate}

A typical growth run started with a substrate previously etched using the high temperature hydrogen furnace. The sample was degreased using acetone and methanol, and subsequently immersed in a HF:Reagent Alcohol = 3:1 (by volume) solution for 10 minutes to remove surface oxides accumulated during the etching process. Once the sample was secured inside the system and the pressure reduced to the desired level, the pre-growth surface chemical composition and crystal structure examination were performed using AES and RHEED. The substrate was then ramped up to the desired growth temperature $\left(700^{\circ} \mathrm{C} / 800^{\circ} \mathrm{C}\right)$. Two temperature measurements were taken here. One was the back thermocouple measurement (BTC), which measured the temperature on the back of the sample holder, the other was taken on the front face of the substrate using an optical pyrometer. Since molybdenum had excellent heat conductivity property, the back thermocouple measurement was a very good indication of the true temperature at the face of the sample holder. This was described in more detail in Section 3.1. It took approximately 7 minutes for the BTC measurement to reach set temperature corresponding to the percentage power output. In the mean time, an infrared pyrometer measurement was taken on the surface of the substrate to determine its actual temperature. Because of a lack of heat conducting media between the substrate and the sample holder, there was an approximately 20-minute time lag until the substrate reached the desired growth temperature (as measured by the optical pyrometer). Since AES, AFM, and RHEED data had confirmed that $6 \mathrm{H}-\mathrm{SiC}$ substrates annealed up to $800^{\circ} \mathrm{C}$ for 90 minutes showed no distinguishable difference before and after annealing, the source gases were not leaked into the 
growth chamber until the sample reached the desired growth temperature. The runs consisted of two different fluence levels that were calculated based on growth time periods of 15 minutes and 60 minutes $($ Flux $\times$ Growth Time $=$ Fluence $)$. Methylsilanes were introduced into the chamber through a Varian leak valve to a predetermined pressure calculated from corresponding flux level (i.e. $0.342 \mathrm{ML} / \mathrm{sec})$.

During the growth period, in-situ analysis of sample was performed once using RHEED. The pressure of the source gas was maintained constant until the end of the growth run when it was shut off before the heater was turned off. The cooling process generally lasted one hour before sample was at an acceptable temperature for both transport and the AES analysis. The sample was then removed from the system to perform other ex-situ analysis of AFM (surface morphology, roughness) and FESEM (film/substrate interface structure and morphology). 


\section{Chapter 4: Results and Discussion}

The goal of this research is to develop methods of growing smooth epitaxial SiC films on Si (001) and 6H-SiC (0001) substrates using novel gas precursors and methods of controlling substrate surface structure. The gas precursors considered here are DMS and TMS. In addition to gas precursor identity, the growth parameters include growth temperature, growth time, and gas flux. Characterization of the pre-growth surface composition, structure, and morphology of both $6 \mathrm{H}-\mathrm{SiC}$ and $\mathrm{Si}$ are discussed in Section 4.1. In the case of $6 \mathrm{H}-\mathrm{SiC}$ substrates, the results of the high temperature H-etching studies to create stepped surfaces are presented. In Section 4.2, homoepitaxial growth runs on $\mathrm{H}$-etched $\mathrm{SiC}$ substrates are discussed. For both MS and DMS, the results are presented in two sub-sections, one for high temperature growth $\left(800^{\circ} \mathrm{C}\right)$ and the other for low temperature growth $\left(700^{\circ} \mathrm{C}\right)$. Each of these sub-sections is further broken into high and low flux growth runs, and these are divided into short (15 minutes) and long (60 minutes) growth runs. In Section 4.3, the findings are discussed for heteroepitaxial $\mathrm{SiC}$ growth on $\mathrm{Si}$ substrates using MS and DMS gas sources. The presentation of results is structured the same as that for homoepitaxial growth runs in Section 4.2.

\subsection{Starting Sample Surfaces}

\subsubsection{Silicon Substrate Pre-Growth Surface}

The starting surface for all $\mathrm{Si}(001)$ substrates was a $1 \times 1 \mathrm{H}$-terminated surface created using an HF-based ex-situ wet chemical etch method known as the Fenner Etch. Ziemer ${ }^{48}$ found that such surfaces enabled the formation of an effective barrier for Si out-diffusion during $\mathrm{SiC}$ growth on Si. This reduction of Si activity was critical in reducing pit/void formation during $\mathrm{SiC}$ 
growth. Consequently the Fenner-etched surface was thought to be a "promising starting point" for the growth of high quality $\mathrm{SiC}$ film on $\mathrm{Si}$. These observations were discussed in detail in Ziemer's work. Their implications were also discussed earlier in this work and will not be

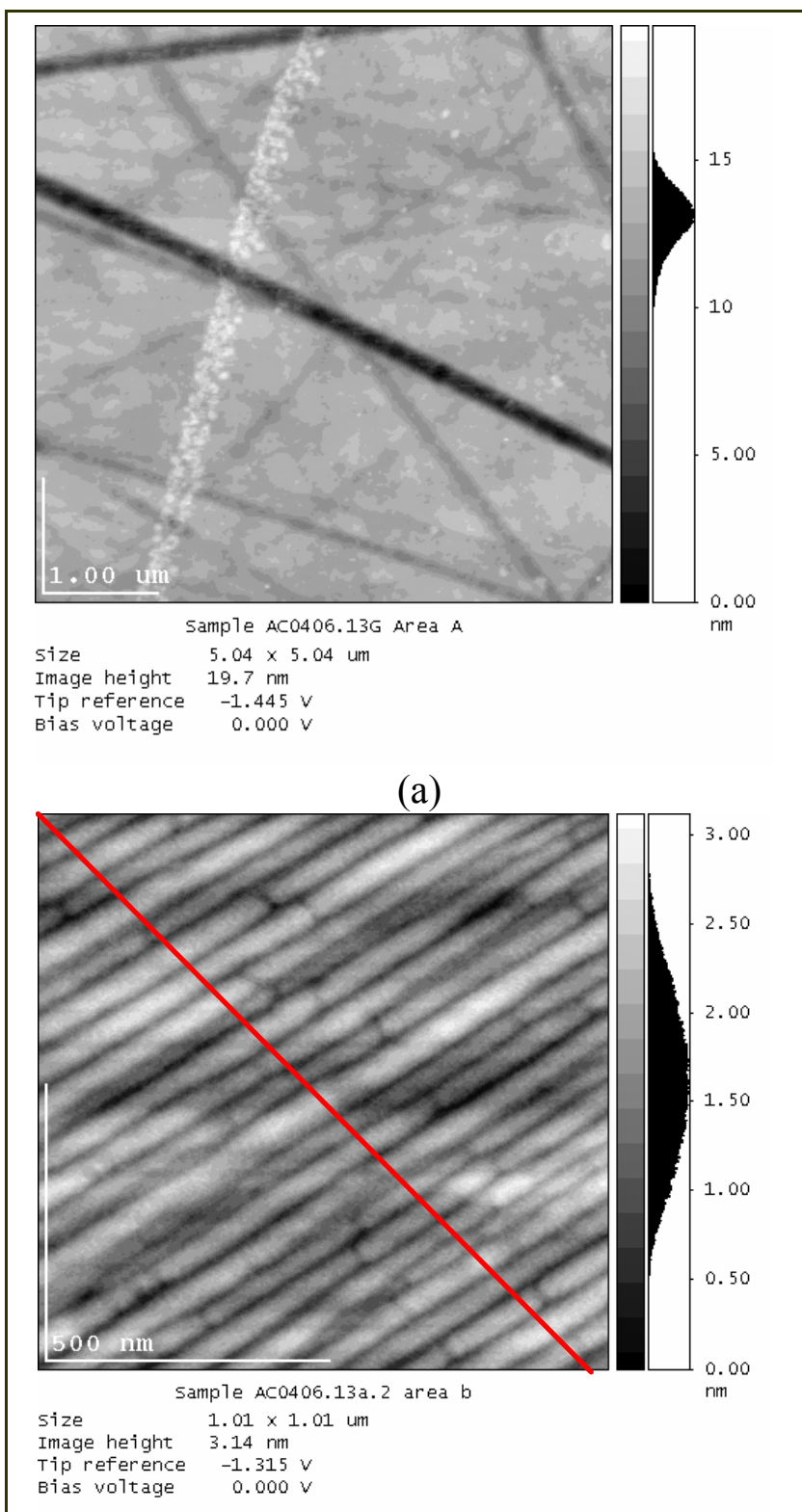

(b)

Figure 4-1. AFM images of $6 \mathrm{H}-\mathrm{SiC}$ substrate as received (a), and after high temperature hydrogen etching (b). further elaborated here.

\subsubsection{H-SiC Substrate Surface}

Commercially available SiC substrates are grown as a 2-inch or 3-inch cylindrical boules, which are then sliced into wafers several hundred of $\mu \mathrm{m}$ in thickness. To produce a smooth surface for electronic applications and further epitaxial growth, the manufacturers polish these wafers using a finegrit diamond paste. The polished wafers are covered with fine scratches of various sizes upon the conclusion of the polishing process. Although invisible to the naked eye, these scratches can be detrimental to electronic applications and ensuing epitaxial growth alike.

Figure 4-1(a) shows an AFM image typical of an as-received $\mathrm{SiC}$ wafer. These scratches were random in direction, up to tens of nanometers deep, and hundreds of 
nanometers wide. SiC substrates used in this research were etched at high temperature in hydrogen to remove the scratches and other subsurface defects associated with the polishing process. After hydrogen etching, sample surfaces displayed ordered steps and terraces. These step heights were about $1.5 \mathrm{~nm}$ and the terrace's width ranged up to $200 \mathrm{~nm}$, depending upon the extent to which wafer surface was cut off-axis. Figure 4-1(b) shows an AFM image of the stepand-terrace morphology typically observed after hydrogen etching. In this case, the wafer that was cut $3.5^{\circ}$ off-axis and the terrace widths are $\sim 30 \mathrm{~nm}$ while the step heights are $\sim 1.5 \mathrm{~nm}$.

The procedure for high temperature hydrogen etching has been described in detail in section 3.5. A quantity of primary significance in these etching studies was the etch rate measured as mass loss per unit area per unit time or as thickness loss per unit time. Since etching occurred during at least part of the temperature ramp, determinations of the etch rate corresponding to a given temperature required two separate runs, one with and another without a dwell time under otherwise equivalent conditions of gas flow rate, gas composition, and etch temperature. Because it was not necessary or desirable to duplicate runs (with and without dwell) for every etch condition, the quantity based on the mass loss per unit area or the equivalent thickness loss (ETL) was frequently used to compare the etch process for the different experimental conditions. The ETL was calculated by dividing the measured mass loss by the density of SiC and the surface area of the sample.

Figure 4-2 shows a plot of ETL versus hydrogen composition for an etch temperature of $1600^{\circ} \mathrm{C}$ with no dwell, and a flow rate of 8 standard cubic foot per hour (SCFH). In the cases of mixed gas, Ar was used the carrier. An ETL of $5.9 \mu \mathrm{m}$ was observed for $100 \% \mathrm{H}_{2}$, and it decreased to $0.9 \mu \mathrm{m}$ for $5 \% \mathrm{H}_{2}$. These data show a clear dependence of the etch process on the hydrogen concentration. 


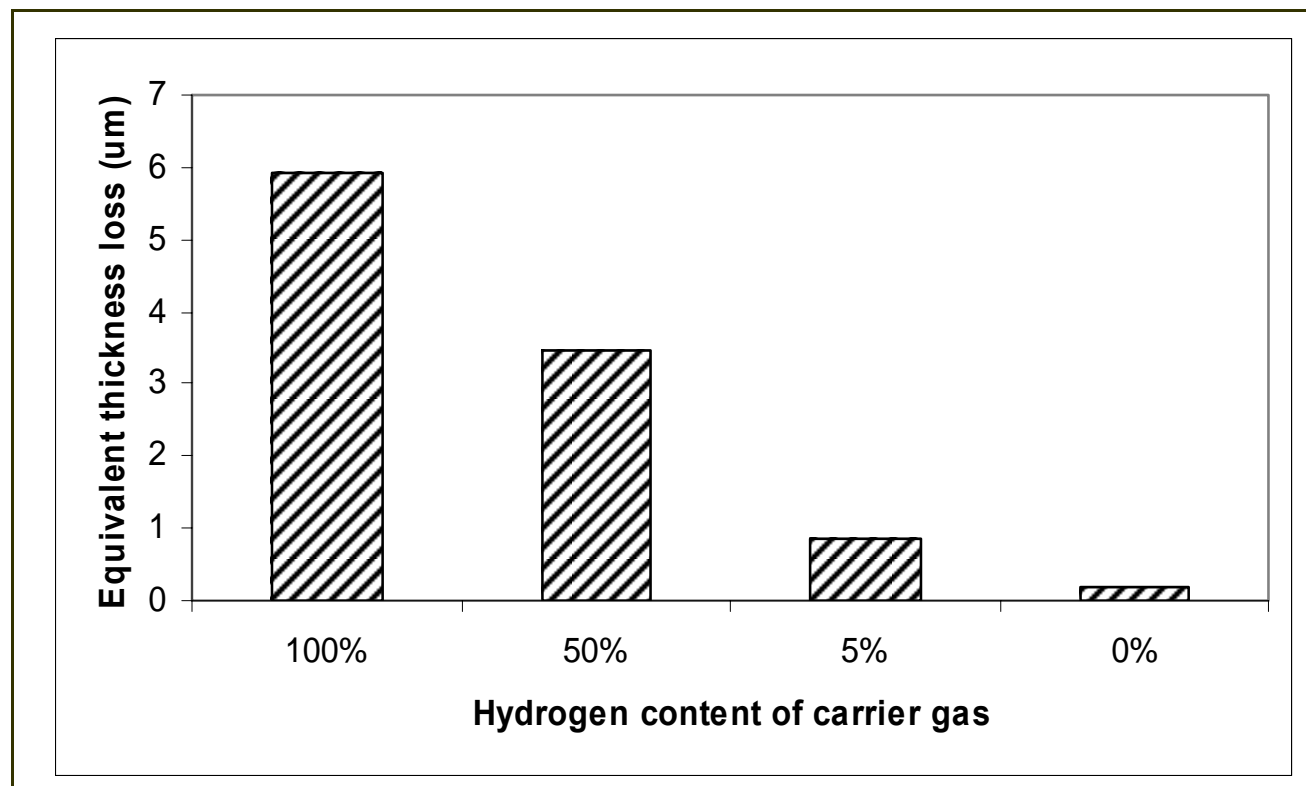

Figure 4-2. Plot of ETL versus $\mathrm{H}_{2}$ concentration for $1600^{\circ} \mathrm{C}$ and a flow rate of $8 \mathrm{SCHF}$. The dwell time for each data point was 0 hours.

As dwell times were increased to 2 and 4 hours at $8 \mathrm{SCFH}$ and $1600^{\circ} \mathrm{C}$ for $100 \% \mathrm{H}_{2}$, ETLs of $21.1 \mu \mathrm{m}$ and $35.7 \mu \mathrm{m}$ were obtained, respectively. Based on these data, an etch rate was of 7.5-7.6 $\mu \mathrm{m} /$ hour was calculated for the conditions of $100 \% \mathrm{H}_{2}, 8 \mathrm{SCFH}$, and $1600{ }^{\circ} \mathrm{C}$.

Similar measurements for $5 \% \mathrm{H}_{2}, 8 \mathrm{SCFH}$, and $1600^{\circ} \mathrm{C}$ showed the etch rate to be between $1.1 \mu \mathrm{m} /$ hour and $1.3 \mu \mathrm{m} /$ hour. These results, illustrated in Figure 4-3, indicate that the etch rate was a function of $\mathrm{H}_{2}$ concentration and that the experiments were reasonably reproducible. The etch rates measured here were comparable to those reported by Masahara. ${ }^{87}$

As expected, the $100 \% \mathrm{H}_{2}$ etch was more aggressive than those using lower $\mathrm{H}_{2}$ concentrations. For all the $1600^{\circ} \mathrm{C}, 100 \% \mathrm{H}_{2}$ runs, regardless of the dwell time and flow rate, the surfaces were covered by what appears to be ridges with occasional hexagonal features (Figure 4-4(a)). These features were particularly obvious for samples with longer dwell times of 2 and 4 hours at $1600^{\circ} \mathrm{C}$ and $8 \mathrm{SCFH}$. 


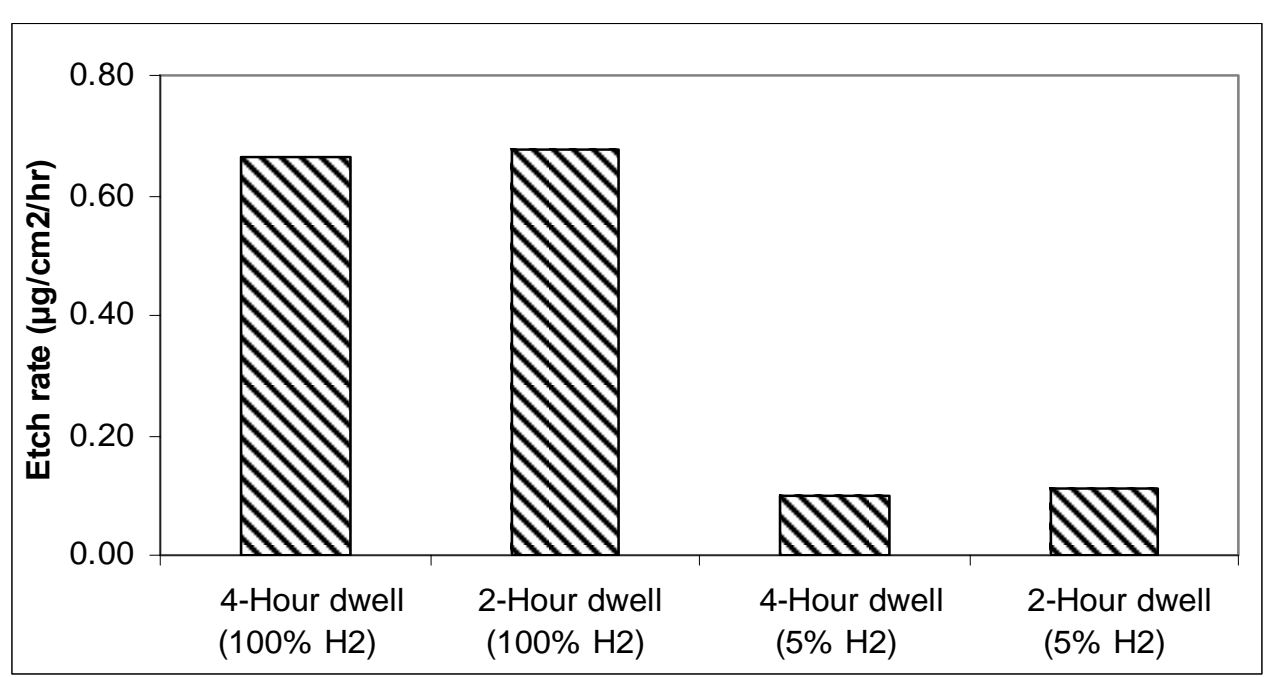

Figure 4-3. SiC etch rate versus gas composition for $1600^{\circ} \mathrm{C}$ and $8 \mathrm{SCFH}$.

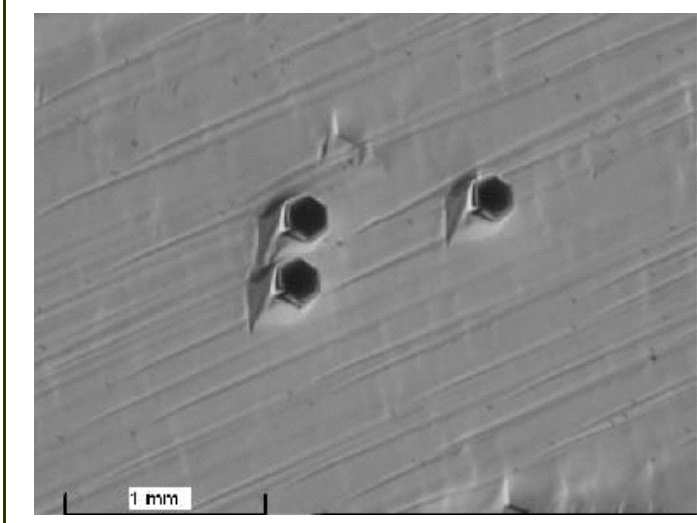

(a)

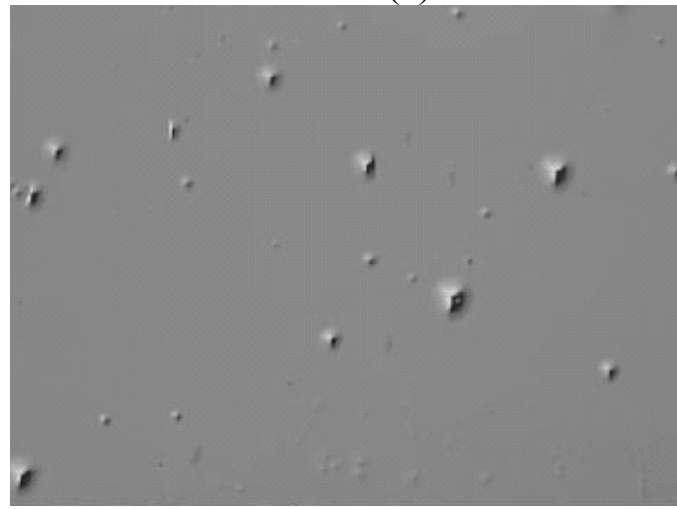

(b)

Figure 4-4. 50× optical micrograph of postetch surfaces produced by (a) $100 \% \mathrm{H}_{2}$, and (b) $5 \% \mathrm{H}_{2}$ with no dwell at $1600^{\circ} \mathrm{C}$.
This rugged surface contrasted markedly with surfaces produced by etching with lower $\mathrm{H}_{2}$ concentrations. This is seen in Figure 4-4 which shows the results of etching at $1600^{\circ} \mathrm{C}$ with no dwell time, $5 \% \mathrm{H}_{2}$ at $8 \mathrm{SCFH}$. These surfaces no longer exhibited the ridges though close-up views $(500 \times$ or higher) still show some hexagonal features. These features were so dissimilar in shapes and location that they raised questions on the effect of pre-etch cleaning procedures. This is discussed more below.

Both etched and un-etched samples were examined using AFM to characterize the surface morphology. The AFM data indicated that all the 
etched surfaces (except for those prepared using pure Argon) clearly displayed arrays of steps and terraces. Table 4-1 details the results in both ETL and AFM data.

Table 4-1. Summary of selected hydrogen-etch runs and results

\begin{tabular}{|c|c|c|c|c|c|c|c|c|c|c|}
\hline \multirow{3}{*}{ Run \# } & & & \multicolumn{4}{|c|}{ Run Conditions } & \multicolumn{4}{|c|}{ Results } \\
\hline & \multicolumn{2}{|c|}{ Sample } & Temperature & $\mathbf{H}_{2}$ & Dwell & Flow & ETL & Terrace & Height & RMS \\
\hline & Designation & Location & $\left({ }^{0} \mathrm{C}\right)$ & $(\%)$ & (hours) & (scfh) & $(\mu \mathrm{m})$ & $(\mathrm{nm})$ & $(\mathbf{n m})$ & $(\mathbf{n m})$ \\
\hline 1 & AZ1023-08 & 5 & 1600 & 100 & 0 & 8 & 5.9 & 100 & 5.0 & 1.7 \\
\hline 2 & AZ1023-08 & 6 & 1500 & 100 & 0 & 8 & 2.1 & 300 & 8.0 & 2.7 \\
\hline 3 & AZ1023-08 & 7 & 1600 & 100 & 0 & 1 & 2.9 & not clear & & 6.2 \\
\hline 4 & AZ1023-08 & 8 & 1600 & 50 & 0 & 8 & 3.5 & 120 & 3.4 & 0.6 \\
\hline 5 & AZ1023-08 & 14 & 1600 & 5 & 0 & 8 & 0.9 & 60 & 1.4 & 0.5 \\
\hline 6 & AZ1023-08 & 15 & 1600 & 100 & 4 & 8 & 35.7 & 80 & 0.1 & 0.5 \\
\hline 7 & AZ1023-08 & 16 & 1600 & 100 & 2 & 8 & 21.1 & 100 & 3.0 & 0.5 \\
\hline 8 & AZ1023-08 & 9 & 1600 & 0 & 0 & 8 & 0.3 & & & \\
\hline 9 & AZ1023-08 & 10 & 1600 & 3 & 0 & 8 & 0.7 & 210 & 2.5 & 0.6 \\
\hline 10 & AZ1023-08 & 11 & 1600 & 5 & 4 & 8 & 5.4 & 300 & 0.1 & 0.5 \\
\hline 11 & AZ1023-08 & 15 & 1600 & 5 & 2 & 8 & 3.4 & 70 & 1.1 & 0.3 \\
\hline 12 & AZ1023-08 & 12 & 1600 & 5 & 0 & 1 & 2.2 & 130 & 5.1 & 1.2 \\
\hline
\end{tabular}

Based on the AFM analyses, the RMS roughness, the step heights, and the terrace widths may be determined. For the etched samples observed in these studies, there is no clear correlation between the measured step heights and the etching conditions. Step heights ranging randomly from $0.08 \mathrm{~nm}$ to $8 \mathrm{~nm}$ were observed. Similar comments can be made concerning the RMS values that range from $0.3 \mathrm{~nm}$ to $6.2 \mathrm{~nm}$ (both of which are of the roughest samples as observed by optical microscopy). Observed terrace widths ranged from 60 to $300 \mathrm{~nm}$.

Figure 4-5 shows an FESEM image of an etched substrate that displays a variety defect features. Some were simple nanometer-scaled holes, while others were elongated features of various widths. In several locations these elongated features were bent at angles of $120^{\circ}$ and so displayed hexagonal characteristics. These were common traits and appeared on almost all etched substrates. 


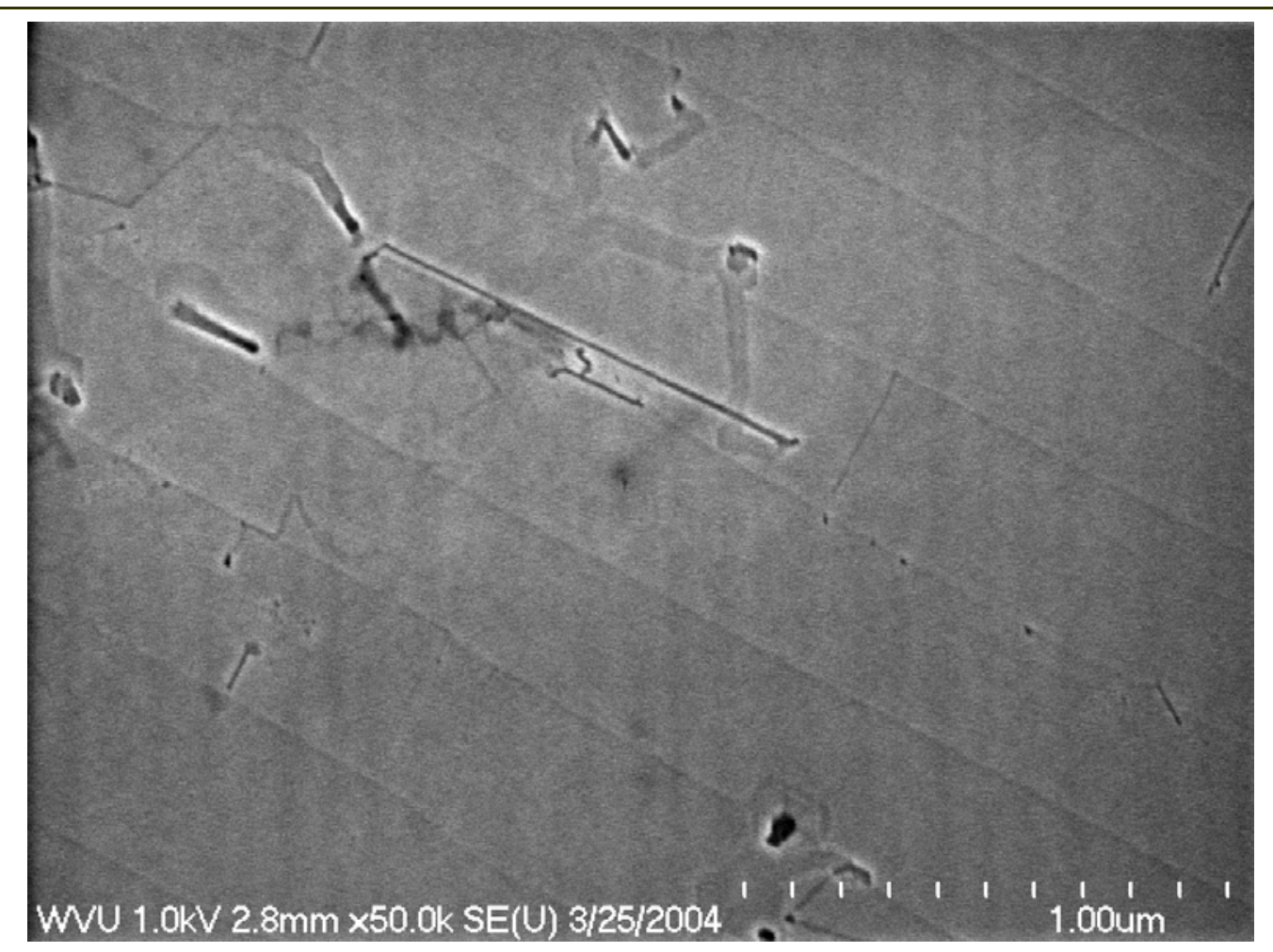

Figure 4-5. FESEM image of substrate etched at $1600^{\circ} \mathrm{C}$ with $5 \%$ hydrogen in $8 \mathrm{SCFH}$ argon carrier gas and no dwell time.

It is unclear at this time whether or not these nano-scaled features identified above are due to pre-existing defects within the wafers, sawing and polishing damage, pre-etch cleaning procedures, or the etching process itself. Although there are always some particles present from the pre-hydrogen-etch cleaning process that cannot be removed under present laboratory conditions, we have never been able to correlate the pre-etch particle density with the defect densities. In addition, there are many extended defect features present at the steps and on the terraces. These are unlikely to be the results of single particles.

Based on an overall assessment of the surface quality and defect level, it was determined that etching at $1600^{\circ} \mathrm{C}$ using $5 \%$ hydrogen in $8 \mathrm{SCFH}$ argon carrier gas with no dwell yielded the lowest level of defect features while still retaining well defined steps and terraces on the surfaces 
of the substrates. These etching conditions were used for all samples prepared for homoepitaxial film growth.

AES analyses were performed to study the surface composition at various stages of the pre-cleaning and etching process. The results of these studies are illustrated by the AES survey spectra shown in Figure 4-6. Upon degreasing (Step 1), as-received SiC wafers had a thick native oxide layer on its surface. The pre-etch HF dip (Step 2) yielded surfaces with lower levels of oxygen contamination. The line-shapes of both the Si-LMM and C-KLL peaks were representative of $\mathrm{SiC}$ with some oxide contamination.

The post-hydrogen-etched surfaces (Step 3) again showed a large amount of surface oxide although thinner than the original oxide layer based on the Si-LMM line shape. This oxide

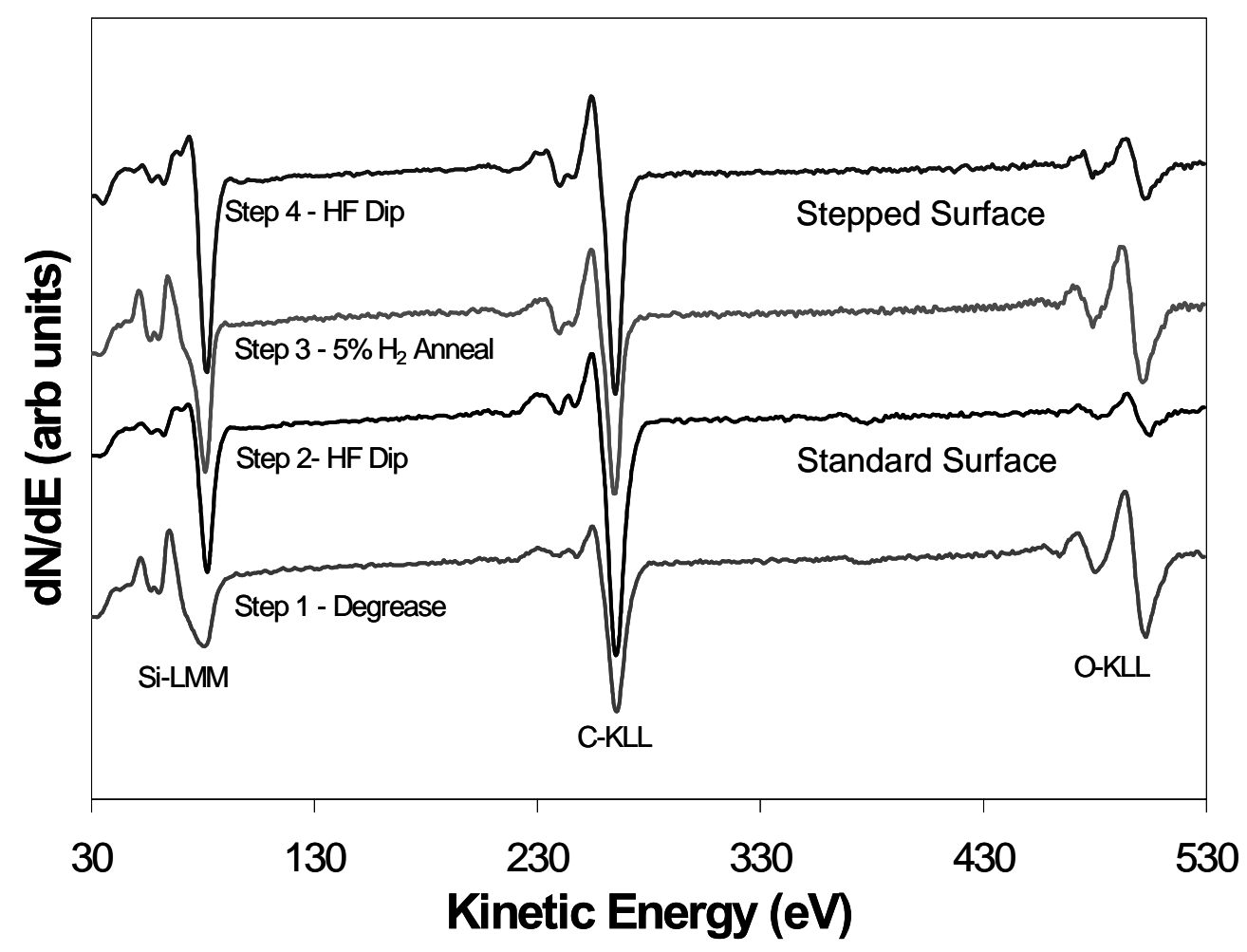

Figure 4-6. AES differentiated spectra of SiC substrate after different steps of the etching process. 
contamination originated from in the UHP gases and impurities generated within the ceramic tube during the etching process, or by interaction with the air while being transported to the analysis system. A post-etch HF treatment (Step 4) was used to remove majority of this oxide. Although we were unable to create a completely oxide-free surface using the available ex-situ sample preparation method, this treatment yielded a reasonably clean surface with well-shaped carbide-like Si-LMM and C-KLL peaks for further SiC homoepitaxial growth.

\subsection{Homoepitaxial Growth on 6H-SiC Substrates}

\subsubsection{High Temperature Homoepitaxial Growth Using MS}

In this set of experiments, films were grown at $800^{\circ} \mathrm{C}$ front face temperature as measured by the optical pyrometer. The MS flux was either $0.2 \mathrm{ML} / \mathrm{sec}$ (low flux) or $1.9 \mathrm{ML} / \mathrm{sec}$ (high flux). Here $1 \mathrm{ML}$ (monolayer) is defined as a fluence (product of flux and time) of $10^{15}$ molecules $/ \mathrm{cm}^{2}$. These growth conditions will simply be abbreviated as HFHT (high flux high temperature) and LFHT (low flux low temperature) in this section. The total growth time was either 15 or 60 minutes. Under low flux (LF) conditions, the total carbon fluence to the samples surfaces was $0.2 \times 10^{3}$ ML for 15 minutes and $0.7 \times 10^{3}$ ML for 60 minutes. Under high flux (HF) conditions, the total carbon fluence was $1.7 \times 10^{3}$ ML for 15 minutes and $6.8 \times 10^{3}$ ML for 60 minutes.

After characterizing the $6 \mathrm{H}-\mathrm{SiC}$ surface with AES and RHEED, the substrate was heated to the growth temperature and the MS pressure (flux) was increased to the desired level and maintained for the desired growth period. The sample temperature and gas precursor was then decreased, and the AES and RHEED analyses were repeated. 
Figure 4-7 shows the Si-LMM and C-KLL spectra before and after the growth under HFHT conditions. To eliminate the effects of variations in Auger instrument operations from one growth run to another, the peak-to-peak intensity of the C-KLL Auger spectra for all pre-

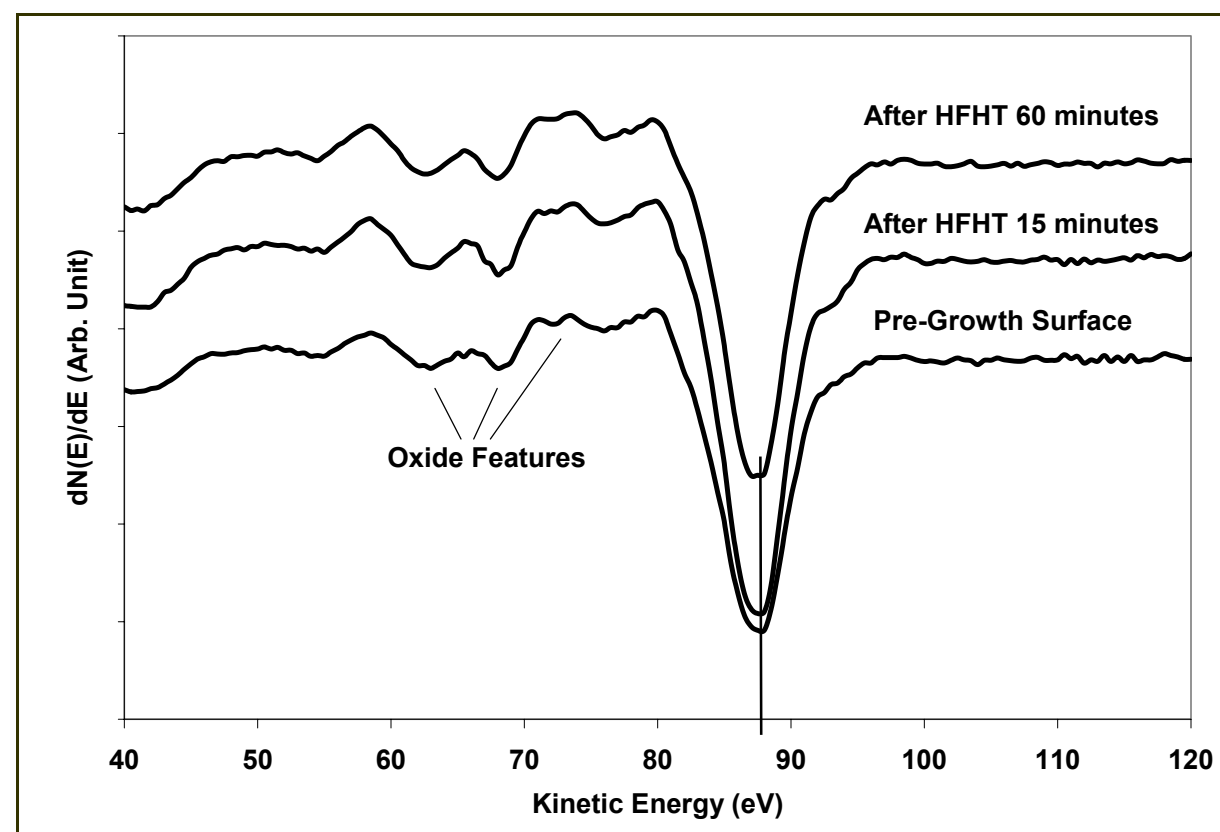

(a)

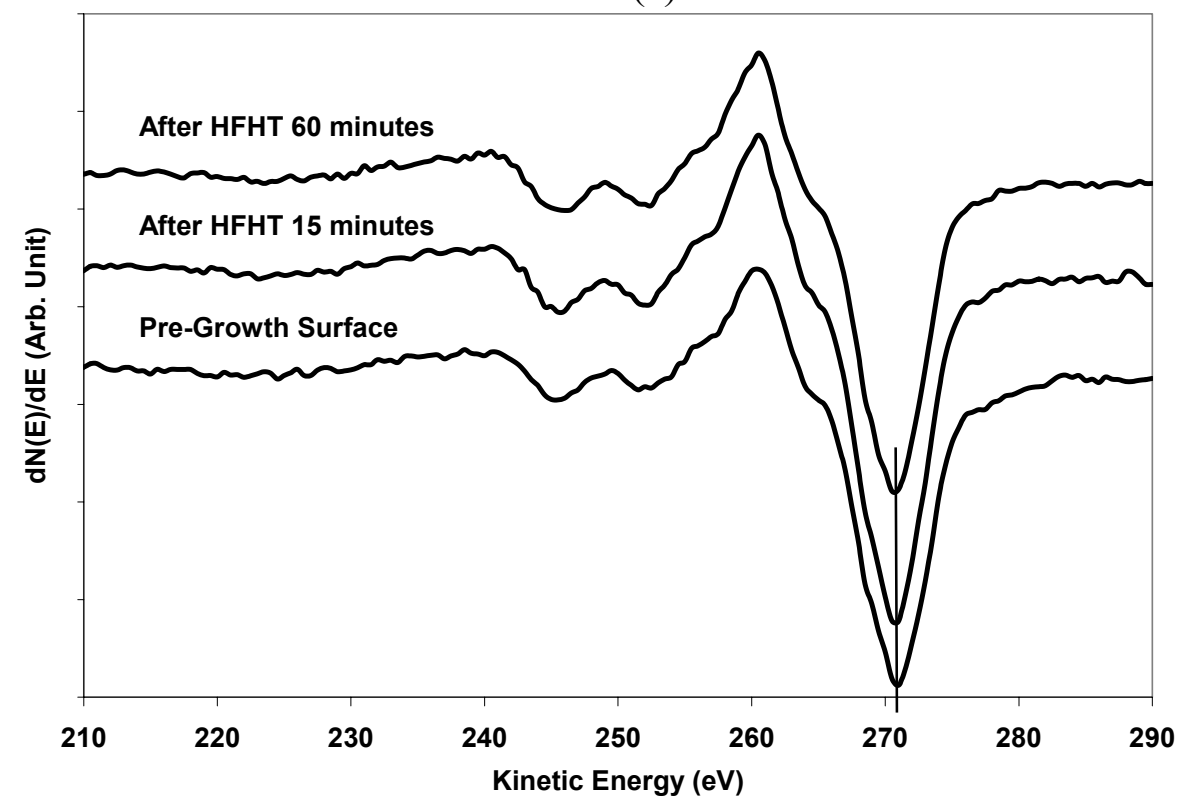

(b)

Figure 4-7. Si-LMM (a) and C-KLL (b) spectra for homoepitaxial growth under HFHT conditions using MS as the gas source. 
growth analyses were referenced back to a single pre-growth C-KLL scan. The ratio of the reference C-KLL peak-to-peak pre-growth intensity to that of a pre-growth C-KLL scan was applied to all Si-LMM and C-KLL scans presented in this study.

The pre- and post-growth AES spectra for both Si-LMM and C-KLL exhibited no distinguishable changes in peak shape, peak-to-peak intensity, position, or width. This suggested that the films grown in this set of the experiments were very similar in chemical composition (i.e. $\mathrm{Si} / \mathrm{C}$ ratio) and bonding to that of the substrates. Alternatively, there may have

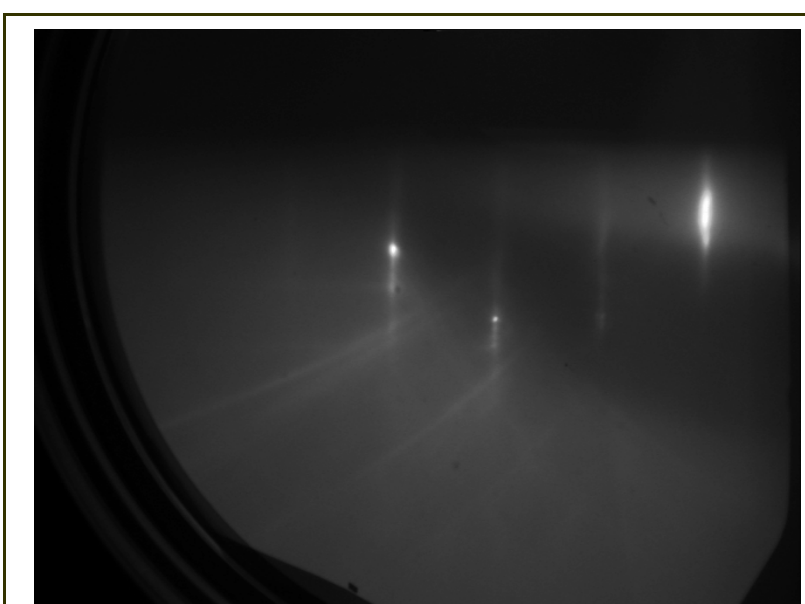

(a)

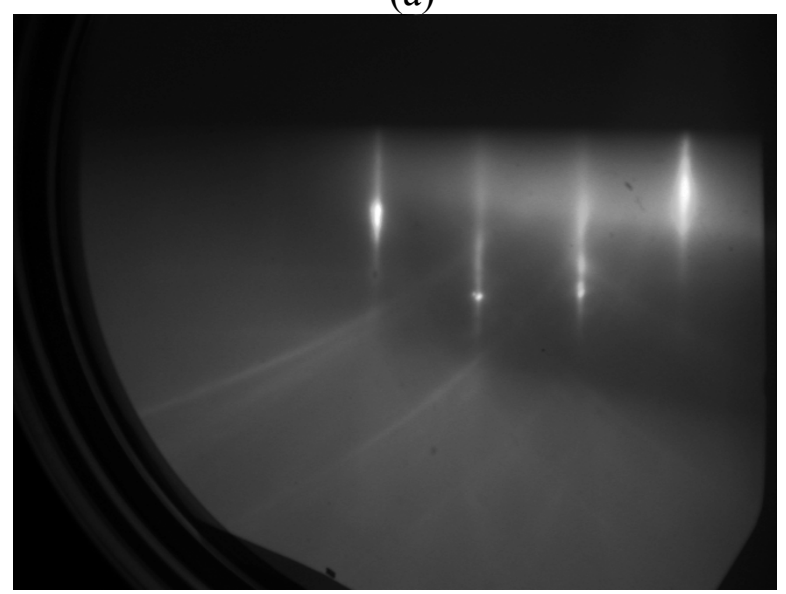

(b)

Figure 4-8. RHEED patterns for $6 \mathrm{H}-\mathrm{SiC}$

substrate before (a) and after (b) 60 minutes of growth using MS under HFHT conditions. been little to no growth at all. The Si-LMM spectra do show the influence of significant oxide $\left(\mathrm{SiO}_{\mathrm{x}}\right)$ features both before and after the growth. The AES spectra for LFHT runs of 15 and 60 minutes displayed the same tendencies and their spectra are not shown here.

RHEED patterns taken before and after the film growth are seen in Figure 4-8. Although these images are for 60-minute growth runs, they are representative of run of 15-minute as well. Analysis of these diffraction patterns indicated that the $\mathrm{d}$ spacing remained same, before and after the HFHT growth. For 6H-SiC (0001) surface, 
the d-spacing is equivalent to the $a$-lattice constant of $5.43 \AA$.

The RHEED patterns, like the AES spectra, suggested two distinct possibilities. First, the films grown on the $6 \mathrm{H}-\mathrm{SiC}$ substrates using MS for both HFHT and LFHT conditions had the same crystal structure and chemical composition as the substrate. Second, there was little film growth under these conditions.

To help determine which one of these scenarios was more likely, the film surface morphology was examined using AFM and FESEM. As illustrated by the images in Figure 4-9 both techniques showed that the steps and terraces on the surface of $6 \mathrm{H}-\mathrm{SiC}$ substrate were still present after film growth.

Based upon the AFM data, two types of RMS roughness $\left(\mathrm{R}_{\mathrm{rms}}\right)$ data were taken. One was that of the entire surface as captured by AFM image (Surface $R_{\text {rms }}$ ), and the other was of the individual terraces (Terrace $\mathrm{R}_{\mathrm{rms}}$ ). This was obtained by performing line profiles on the terraces parallel to the step direction. When there were obvious "glitches" in the AFM images, the glitches were cropped out before obtaining Surface $R_{\text {rms }}$ values. The average values were then computed.

The results of these analyses for of HFHT growth conditions were shown in Figure 49(d). The error bars shown for both $R_{r m s}$ and step heights were based on the percentage error (ratio of standard deviation to mean value). The terrace $\mathrm{R}_{\mathrm{rms}}$ remained in effect unchanged statistically, and this trend held true for the LFHT growth runs as well. Since the AFM scans treated the steps as individual peaks rather than flat plateaus, the RMS roughness of the entire imaged surface would always be larger than that of individual terraces as expected. It was clear Terraces $R_{r m s}$ would be a better indication of the sample's roughness than Surface $R_{r m s}$ here for our analytical purposes. 
A more detailed AFM characterization revealed that the step heights decreased to $0.7 \mathrm{~nm}$ after the 15 minutes of HFHT growth and to $0.8 \mathrm{~nm}$ after the 60 minutes of HFHT growth. That is, all the change in step height occurred in the first 15 minutes of growth. A similar result was obtained for the 60-minute LFHT growth, where the step height decreased to $0.8 \mathrm{~nm}$. For the

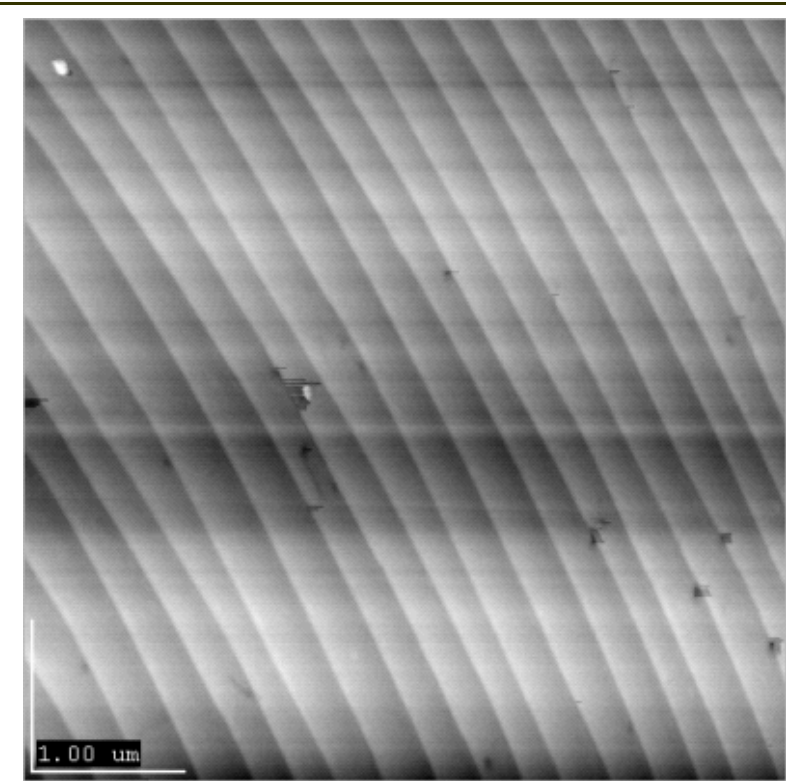

(a)

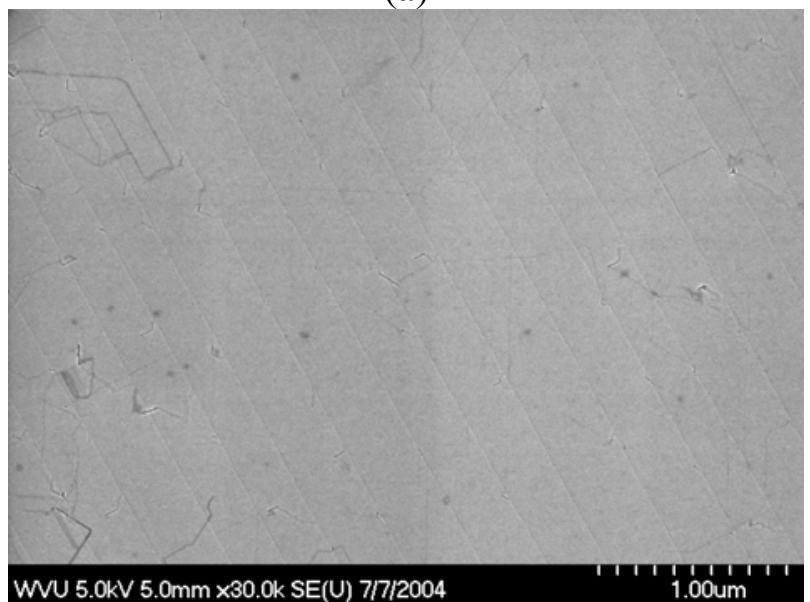

(c)

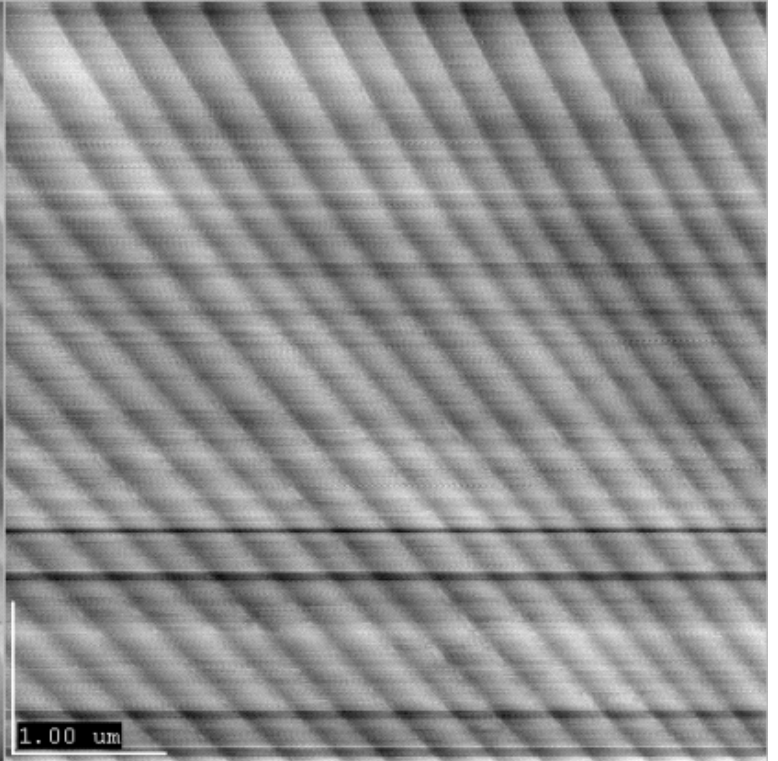

(b)

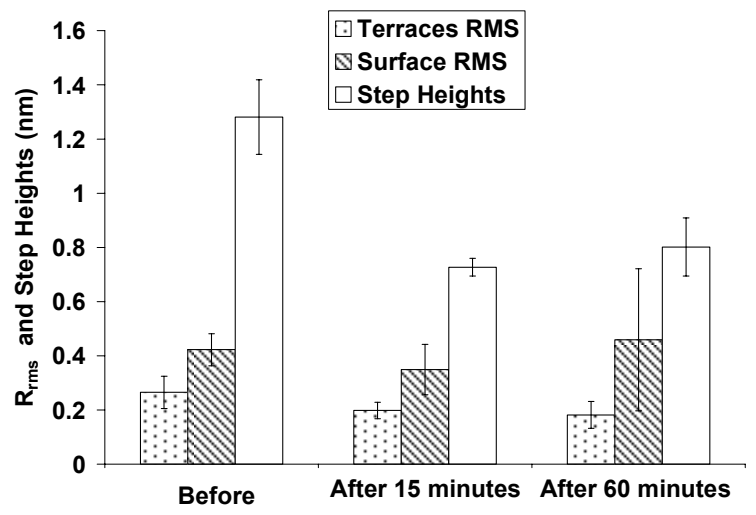

(d)

Figure 4-9. AFM images of the $6 \mathrm{H}-\mathrm{SiC}$ substrate before growth (a) and after 60 minutes of growth using MS under HFHT conditions (b); FESEM image of the film after 15 minutes of growth using MS under HFHT conditions (c); Surface roughness and step height data before and after film growth using MS under HFHT conditions (d). 
15-minute LFHT growth run, however, the step heights remained statistically unchanged.

Growth on the stepped $6 \mathrm{H}-\mathrm{SiC}$ (0001) ( $\mathrm{Si}$ face) is expected to proceed by a step-flow growth mechanism. In this process, growth initiates from the step face and proceeds laterally across the surface. As the name implies, the steps appear to flow across the surface. Normally, one would expect this to leave the step height unchanged as each terrace would be covered by film of same thickness, but incomplete step flow growth across the terrace would result in a decrease in step height. Alternatively, step bunching may results in a reduction of step height.

For $6 \mathrm{H}-\mathrm{SiC}$, Komoto et al. have shown that step bunching plays an important role in epitaxial layer grown by CVD. ${ }^{88}$ For the (0001)-Si face of $6 \mathrm{H}-\mathrm{SiC}$, three $\mathrm{Si}-\mathrm{C}$ bilayer-height steps are the most common ( $\sim 90 \%$ probability). One and six Si-C bilayer-height steps are also present with much lower probability ( $\sim 4 \%$ and $\sim 6 \%$, respectively). The detailed step height probabilities were shown to depend on $\mathrm{C} / \mathrm{Si}$ ratio in the gas source for the CVD process. This of course would be reflected by the $\mathrm{C} / \mathrm{Si}$ ratio of the adsorbed growth species; however, an assessment of this is beyond the scope of the present discussion. The point here is simply that if the substrate is dominated by unit cell steps (there are $6 \mathrm{Si}-\mathrm{C}$ bilayer-height steps per unit cell) before growth, then after growth, the step height may reasonably be expected to decrease.

This reduction in step height is precisely what was observed as shown by the statistical data in Figure 4-9(d). This supported by the AFM images shown in Figure 4-10. Figure 4-10(a) shows a high magnification image of the initial (H-etched) surface which is dominated by unit cell (6 Si-C bilayers) step heights. Figure 4-10 (b) shows a comparable image after 15 minutes of growth using MS under HFHT conditions. Note the appearance of wave-like step flow growth on terraces. 


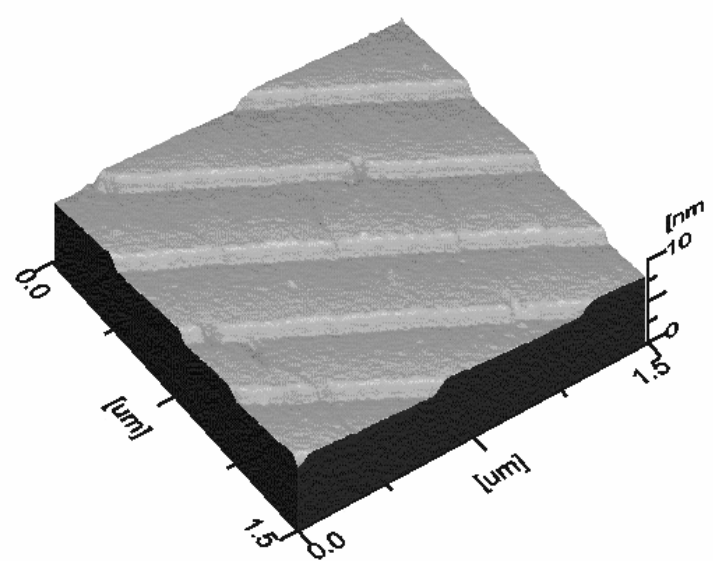

(a)

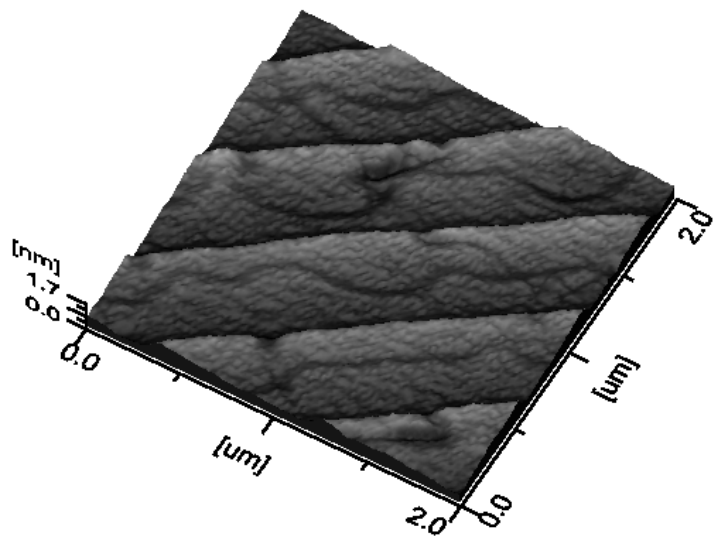

(b)

Figure 4-10. AFM images for $6 \mathrm{H}-\mathrm{SiC}$ substrate before (a) and after (b) 15 minutes of growth using MS under HFHT conditions.

Selected substrates were cleaved using a diamond scribe and broken into two halves. Examination of cross-sections using FESEM revealed no indication of the film-substrate interface.

\subsubsection{Low Temperature Homoepitaxial MS Growth}

In this set of experiments, films were grown at $700^{\circ} \mathrm{C}$ front face temperature as measured by the optical pyrometer. The MS flux was either $0.2 \mathrm{ML} / \mathrm{sec}$ (low flux) or $1.9 \mathrm{ML} / \mathrm{sec}$ (high flux). The total growth time was either 15 or 60 minutes. These growth conditions will simply be abbreviated as HFLT (high flux low temperature) and LFLT (low flux low temperature) in this section. The total growth time was either 15 or 60 minutes. Under low flux (LF) conditions, the total carbon fluence to the samples surfaces was $0.2 \times 10^{3} \mathrm{ML}$ for 15 minutes and $0.7 \times 10^{3} \mathrm{ML}$ for 60 minutes. Under high flux (HF) conditions, the total carbon fluence was $1.7 \times 10^{3} \mathrm{ML}$ for 15 minutes and $6.8 \times 10^{3}$ ML for 60 minutes. 
The growth and analyses sequence was exactly the same as that described in the previous section. Figure 4-11 shows the Si-LMM and C-KLL spectra before and after the growth under HFLT conditions. The pre- and post-growth spectra show no distinguishable changes in peak shape, intensity, position, and width.

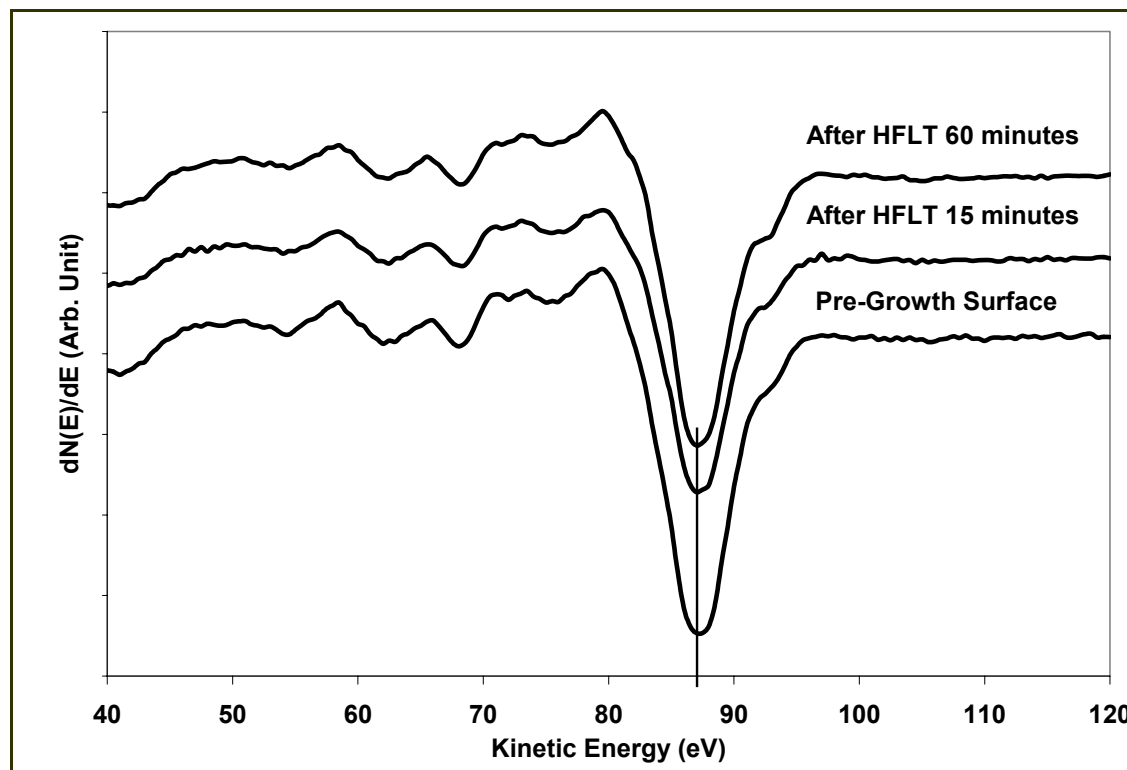

(a)

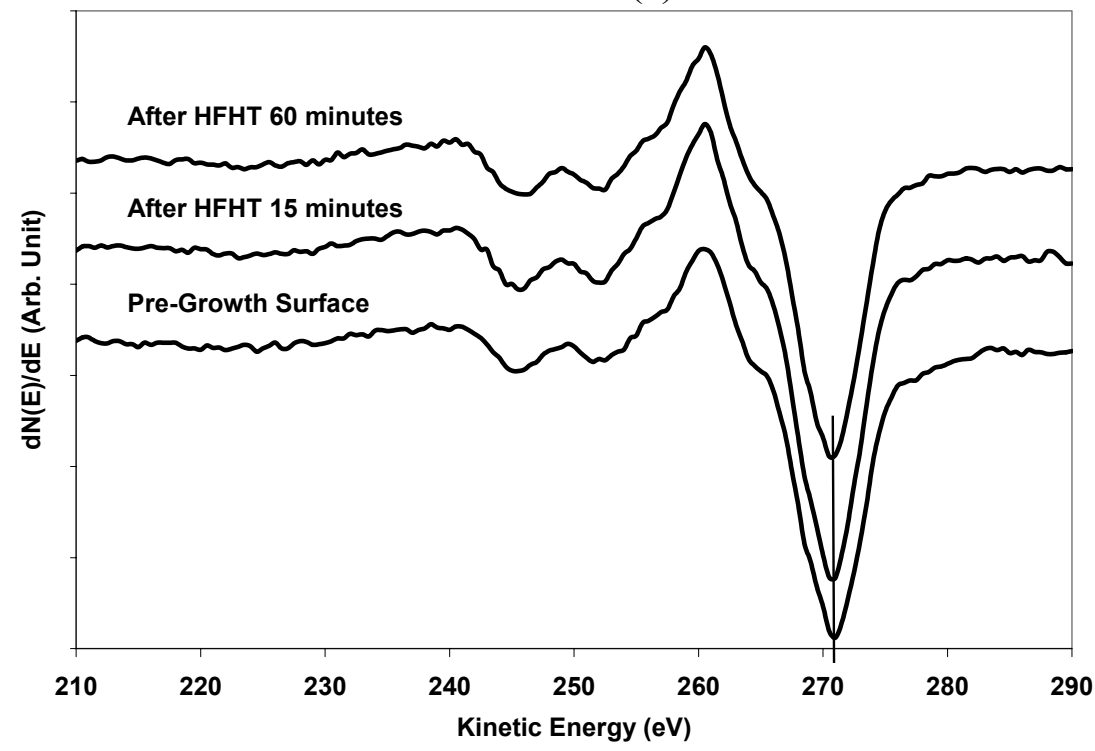

(b)

Figure 4-11. Si-LMM (a) and C-KLL (b) spectra for homoepitaxial growth under HFLT conditions using MS as the gas source. 
As in the HFHT cases, the films grown in this set of the experiments were very similar to the substrate in chemical composition and bonding. There is little if any evidence of $\mathrm{sp}^{2}$ and $\mathrm{sp}^{3}$ C-C bond formation. As before, the Si-LMM spectra show the slight effects of Si-O bond formation. RHEED patterns showed that d-spacing remained unchanged after each growth run. The low flux runs of 15 and 60 minutes displayed the same tendencies and their spectra are not shown here.

AFM and FESEM images shown in Figure 4-12(a)-(c) revealed that the flat terraces on the $6 \mathrm{H}-\mathrm{SiC}$ substrate created by high temperature hydrogen etching were still present after growth.

As observed for the high temperature growth runs, AFM analysis shown in Figur 4-11(d), the Terrace or Surface $\mathrm{R}_{\mathrm{rms}}$ were not statistically different after growth under HFLT conditions.

AFM analysis of the average step height revealed a decrease to $1.2 \mathrm{~nm}$ after the 15 minute HFLT growth run and $1.0 \mathrm{~nm}$ after the 60-minute growth. For the 15-minute and 60minute LFLT growth runs, however, the step heights remained statistically unchanged. The decrease in step height under HFLT conditions again suggests that step-flow growth has taken place with the step bunching mechanism leading to $3 \mathrm{Si}-\mathrm{C}$ bilayer steps. As for the LFLT conditions, nothing definite can be stated, but the absence of the and 3-bilayer steps suggests that limited grown occurred under LFLT conditions.

Selected substrates were cleaved using a diamond scribe and broken into two halves. Examination of cross-sections using FESEM revealed no indication of the film-substrate interface. 


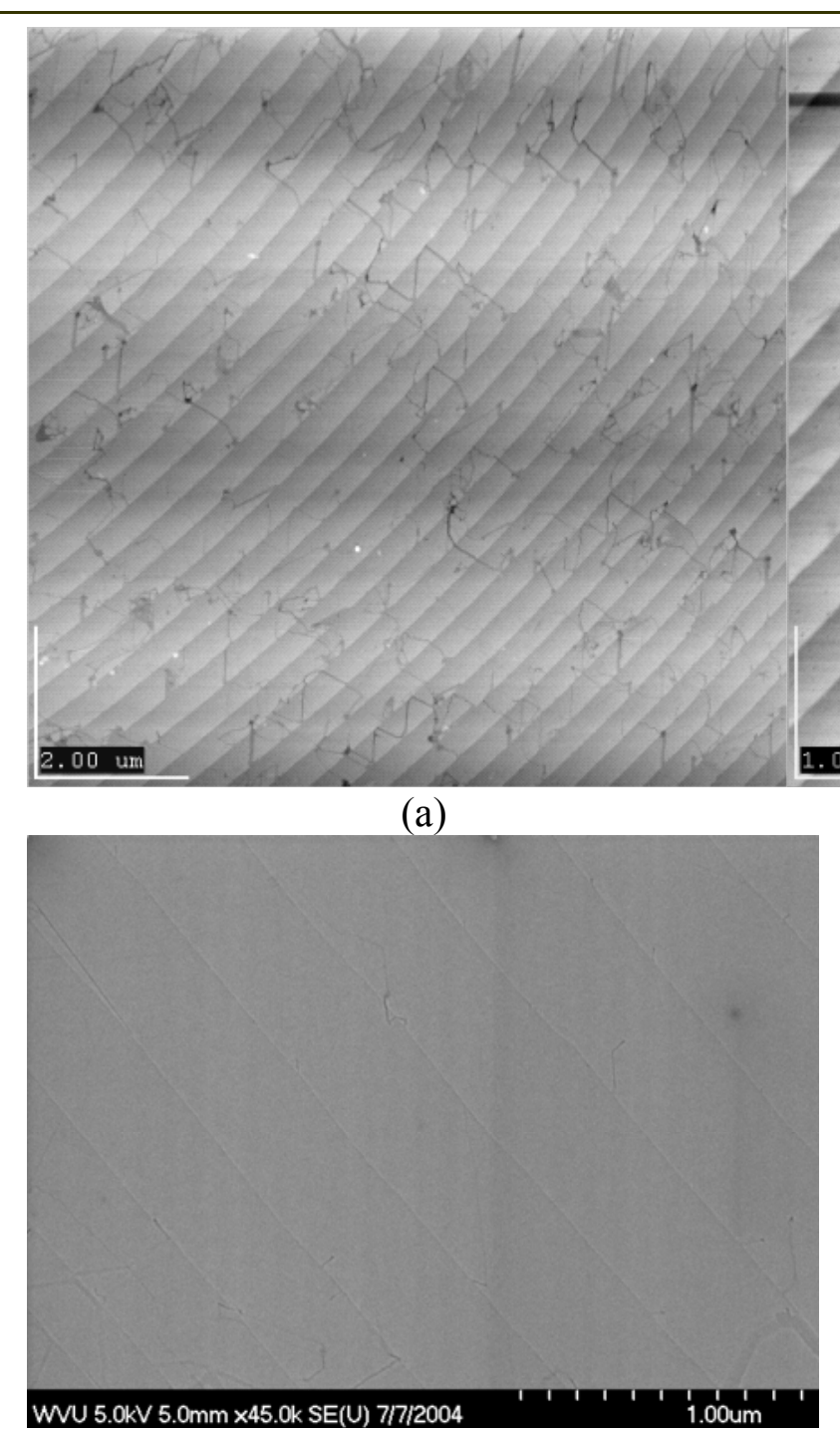

(c) (b)

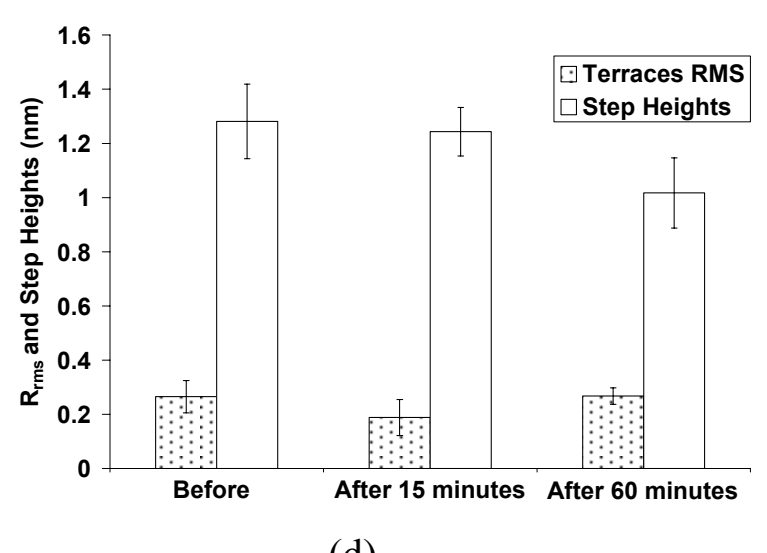

(d)

Figure 4-12. AFM images of the 6H-SiC substrate before growth (a) and after 15 minutes of growth using MS under LFLT conditions (b); FESEM image of the film after 60 minutes of growth using MS under HFLT conditions (c); Surface roughness and step height data before and after film growth using MS under HFLT conditions (d).

\subsubsection{High Temperature Homoepitaxial DMS Growth}

In this set of experiments, films were grown at $800^{\circ} \mathrm{C}$ front face temperature as measured by the optical pyrometer. The DMS flux was either $0.2 \mathrm{ML} / \mathrm{sec}$ (low flux) or $1.9 \mathrm{ML} / \mathrm{sec}$ (high flux). These growth conditions will simply be abbreviated as HFHT (high flux high 
temperature) and LFHT (low flux low temperature) in this section. The total growth time was either 15 or 60 minutes. The carbon flux and fluence in these runs were twice that of MS under otherwise similar conditions.

Figure 4-13 shows the Si-LMM and C-KLL spectra before and after the growth under

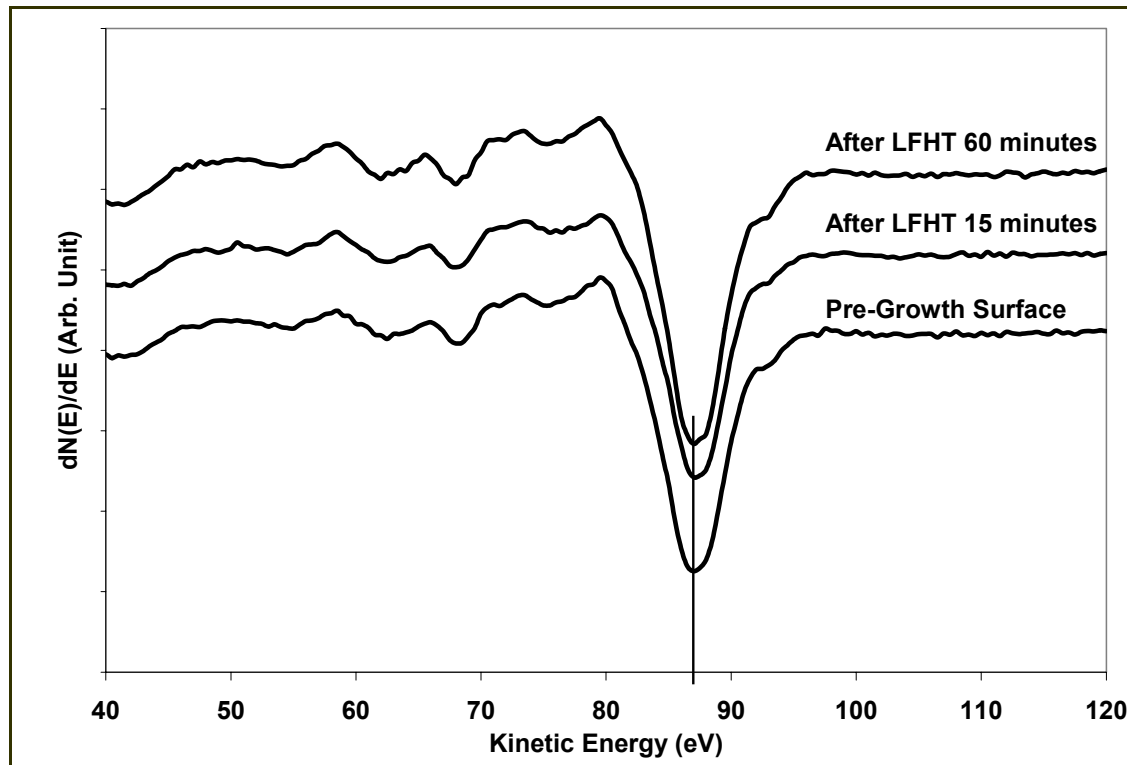

(a)

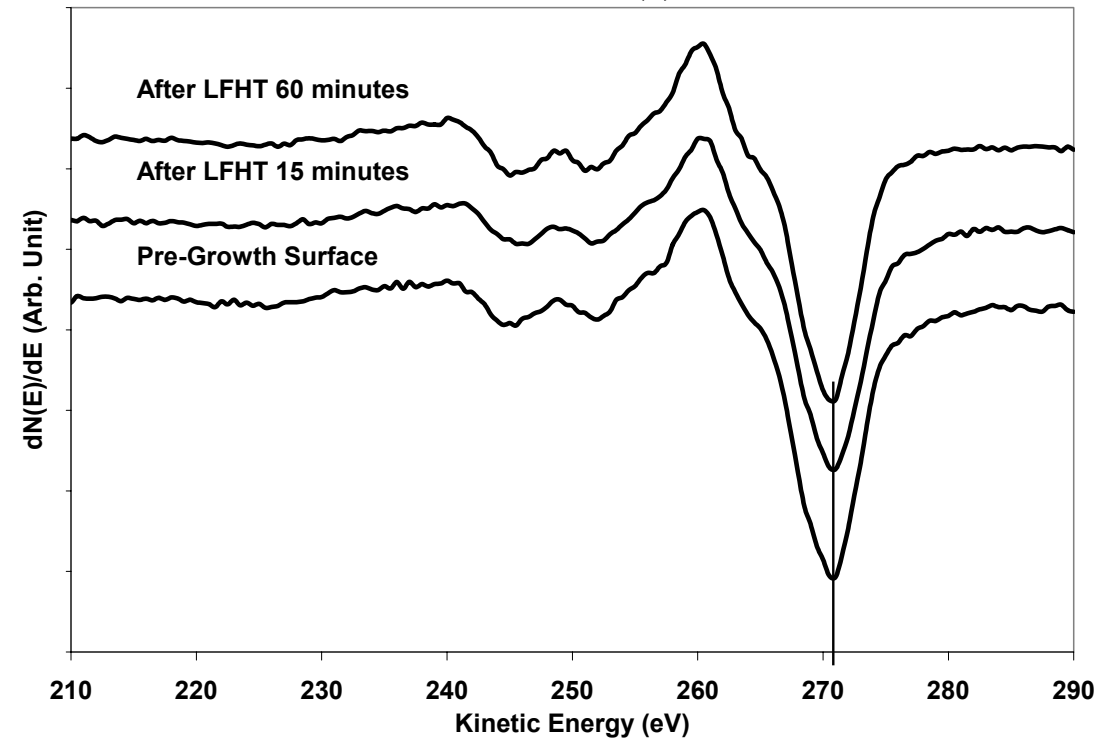

(b)

Figure 4-13. Si-LMM (a) and C-KLL (b) spectra for homoepitaxial growth under LFHT conditions using DMS as the gas source. 


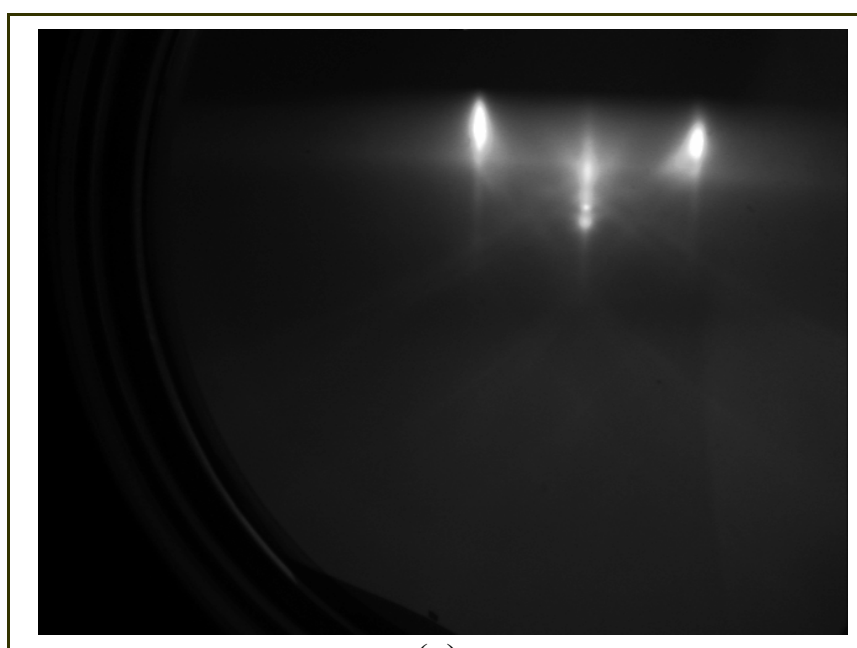

(a)

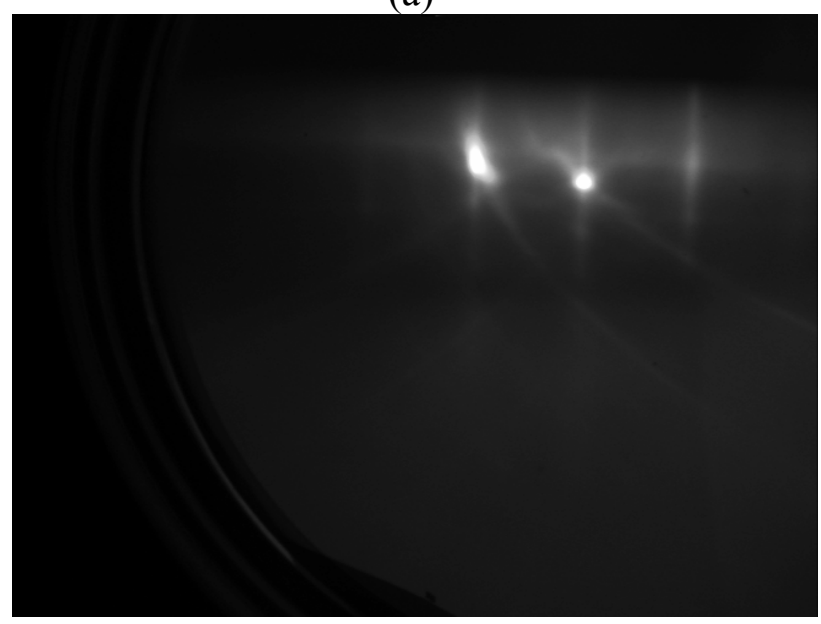

(b)

Figure 4-14. RHEED patterns for $6 \mathrm{H}-\mathrm{SiC}$ substrate before (a) and after (b) 15 minutes of growth using DMS under HFHT conditions.
LFHT conditions using DMS. The pre- and post-growth peak line shapes for both the Si-LMM and C-KLL had no distinguishable changes in peak shape, intensity, position, and width. Similar to the MS studies, this suggests that the films grown in this set of experiments were very similar to the substrate in chemical composition and bonding. AES spectra for the HFHT runs using DMS displayed the same trends.

RHEED patterns taken before and after the growth are seen in Figure 4-14. For both 15-minute and 60-mintue runs using DMS under HFHT conditions, the distance between streaks in RHEED patterns remained unchanged and showed

that the in-plane atomic spacing remain unchanged after film growth. However, the RHEED pattern did appear more spotty after growth, suggesting 3-D type of film growth occurring. AFM data discussed below corroborate this. RHEED images of the 60-minute HFHT and LFHT growth runs were also virtually identical before and after growth.

As seen in Figure 4-15(a)-(c), AFM and FESEM images showed that the step-and-terrace morphology of the surface of $6 \mathrm{H}-\mathrm{SiC}$ substrate was still present after film growth. In contrast to 
the situation for MS, however, clusters rather than step flow appears to be a significant growth mode for DMS under HFHT conditions after 15 minutes. This is illustrated by the triangularshaped features seen in Figure 4-15 (b).

These features had plateau-like structure with heights in the range of $5 \sim 12 \mathrm{~nm}$. Similar but smaller features were found on sample grown for 15 minutes under LFHT conditions. The

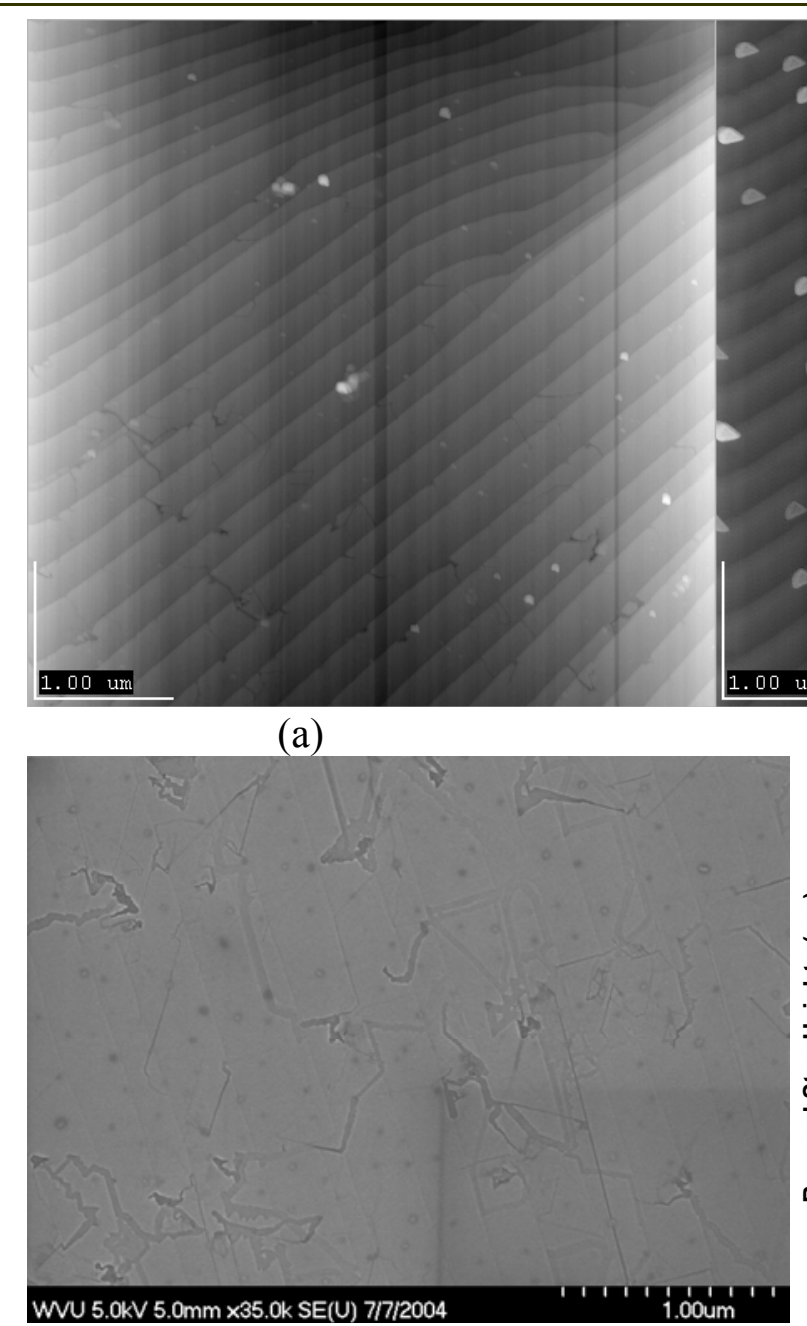

(c)

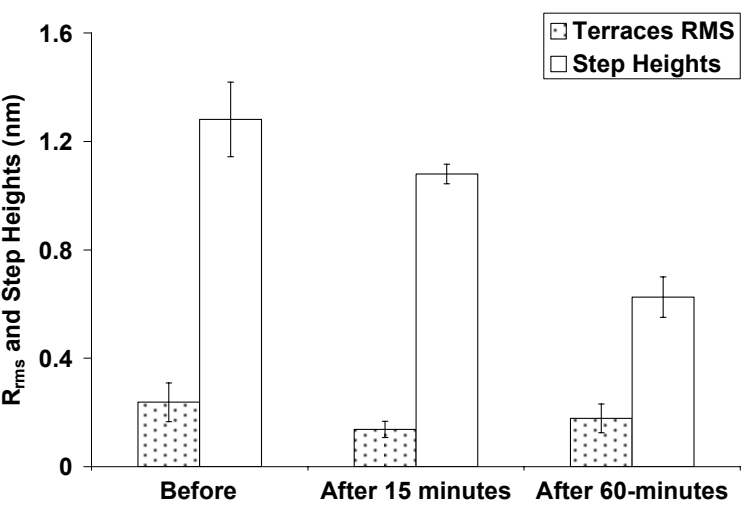

(d)

Figure 4-15. AFM images of the $6 \mathrm{H}-\mathrm{SiC}$ substrate before growth (a) and after 15 minutes of growth using DMS under HFHT conditions (b); FESEM image of the film after 15 minutes of growth using DMS under LFHT conditions (c); Surface roughness and step height data before and after film growth using DMS under HFHT conditions (d). 
presence of these 3-dimensional features on the substrate surface is consistent with what was observed on the RHEED post-growth spotty patterns (Figure 4-14 (b)).

Further characterization of the surface using AFM data showed the average step heights decrease to $1.1 \mathrm{~nm}$ after 15 minutes of HFHT film growth, and a similar decrease was observed after 15 minutes of LFHT growth. These changes in step height are not statistically significant and suggest that when 3-D growth is operative, step flow growth is not. Also, examination of cross-sections using FESEM revealed no indication of the film-substrate interface.

It should be noted that these 3-D features were anomalies. They were observed only for 15 minutes of growth under HFHT and LFHT conditions, and there were no occurrences of such features under any other growth conditions. In fact, upon extending the HFHT and LFHT growth runs to 60 minutes, the 3-D clusters were no longer observed, and though the 60 -minute LFHT run showed no change in step height, the 60-minute HFHT run showed a decrease in step height to $0.6 \mathrm{~nm}$. That is, the step flow growth mode appeared to have re-established at least for the 60-minute HFHT conditions.

The reduction in step height is precisely what was observed as shown by the statistical data in Figure 4-15. This is supported by the AFM images shown in Figure 4-15. Figure 4-16(a) shows a high magnification image of the initial (H-etched) surface, which is dominated by unit cell (6 Si-C bilayers) step heights. Figure 4-16(b) shows a comparable image after 60 minutes of growth using DMS under HFHT conditions. Not the appearance of $3 \mathrm{Si}-\mathrm{C}$ bilayer steps (i.e. half the unit cell step). 


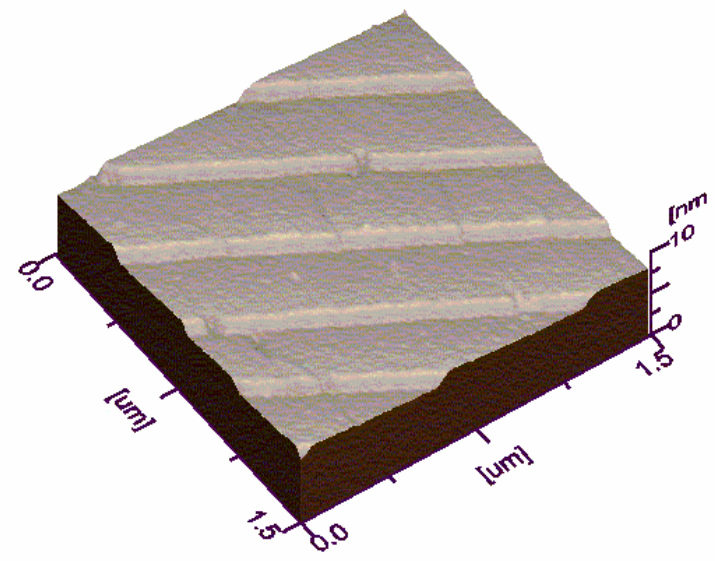

(a)

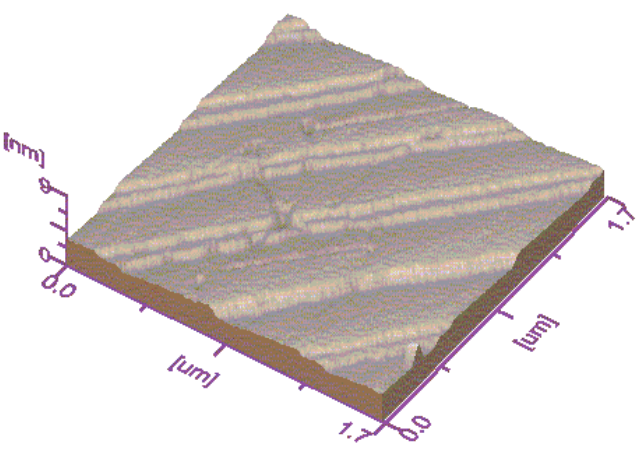

(b)

Figure 4-16. AFM images for 6H-SiC substrate before (a) and after (b) 15 minutes of growth using DMS under HFHT conditions.

\subsubsection{Low Temperature Homoepitaxial DMS Growth}

In this set of experiments, films were grown at $700^{\circ} \mathrm{C}$ front face temperature as measured by the optical pyrometer. The DMS flux was either $0.2 \mathrm{ML} / \mathrm{sec}$ (low flux) or $1.9 \mathrm{ML} / \mathrm{sec}$ (high flux). Growth conditions will simply be abbreviated as HFLT (high flux high temperature) and LFLT (low flux low temperature) in this section. The total growth time was either 15 or 60 minutes. The total carbon fluence was twice that of MS under otherwise similar conditions.

Figure 4-17 shows the Si-LMM and C-KLL spectra before and after the growth under HFHT conditions using DMS. The pre- and post-growth AES spectra for both Si-LMM and CKLL revealed no distinguishable changes in peak shape, intensity, and width, and there were no peak position shifts. The C-KLL also showed no evidence of $\mathrm{sp}^{2}$ and $\mathrm{sp}^{3}$ bonding and retained characteristics of SiC line shapes. The LFLT growth runs of 15 and 60 minutes displayed same 
trend, and their post-growth peak line shapes are not shown here. These observations were consistent with all of the MS growth runs and with the DMS high temperature runs.

RHEED patterns before and after the film growth were examined, and no differences in d-spacing or patterns were observed. These results were also consistent with those for the MS

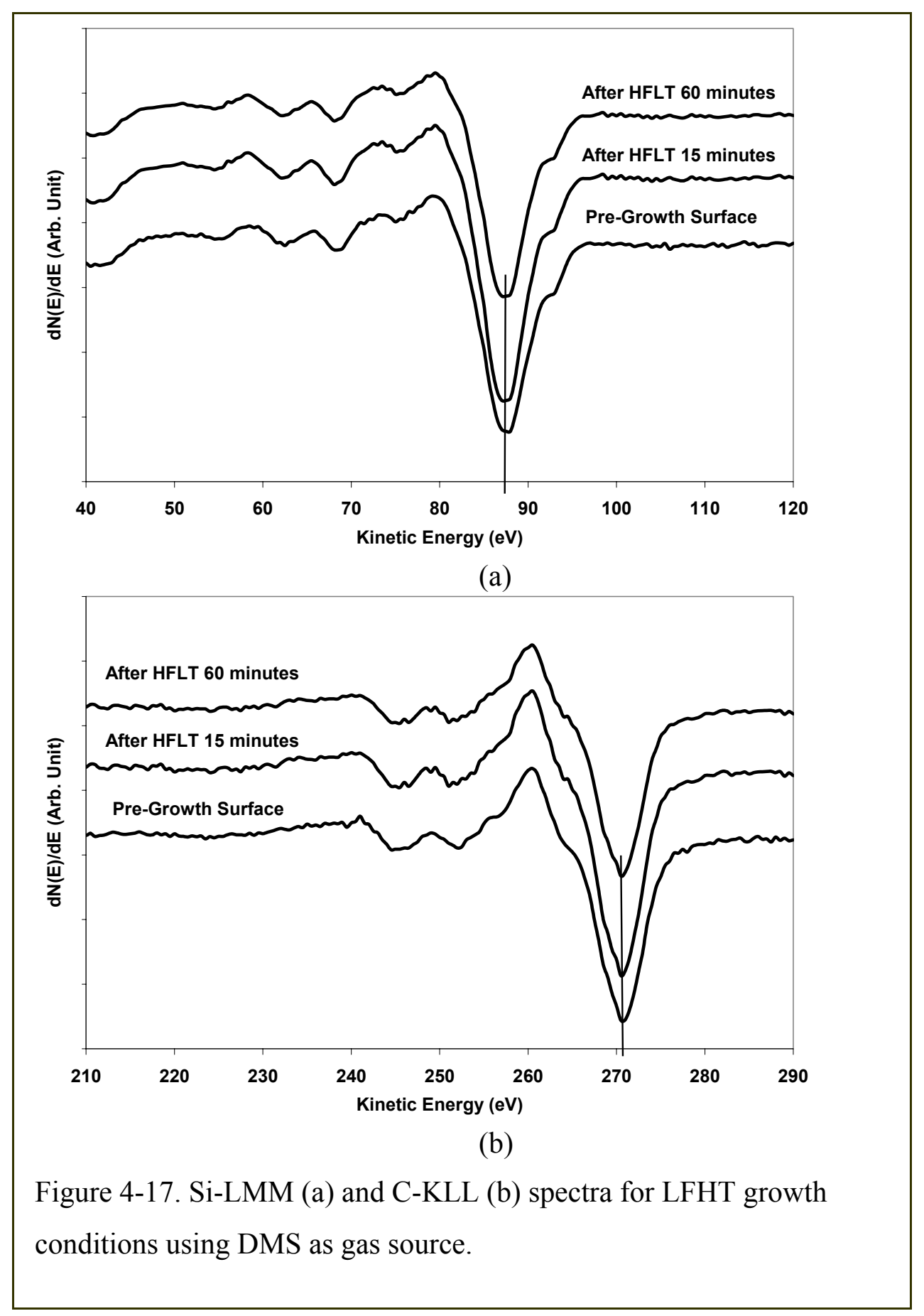


growth runs and the DMS high temperature growth runs.

Figure 4-18 (a)-(c) shows that atomic steps were still present after HFLT growth runs as in all previous homoepitaxial growth runs, regardless of gas source or growth conditions. Although evidences of film growth were suggested by step-growth phenomena, the thin films

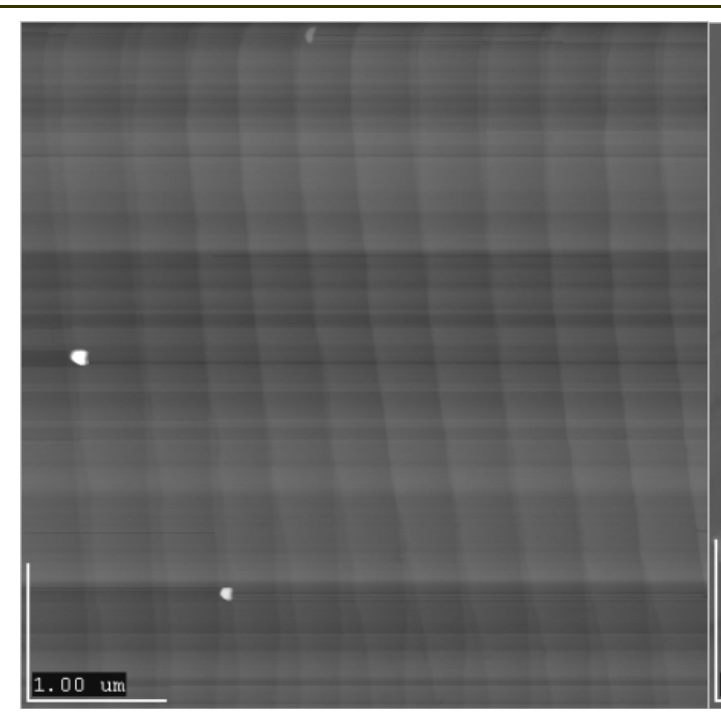

(a)

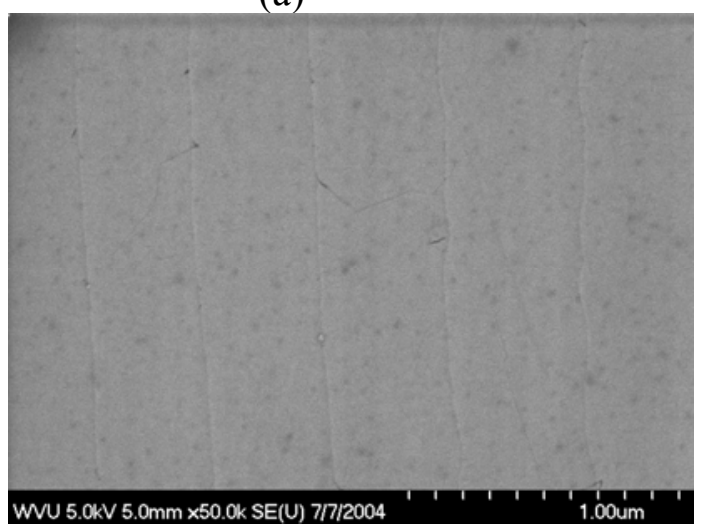

(c)

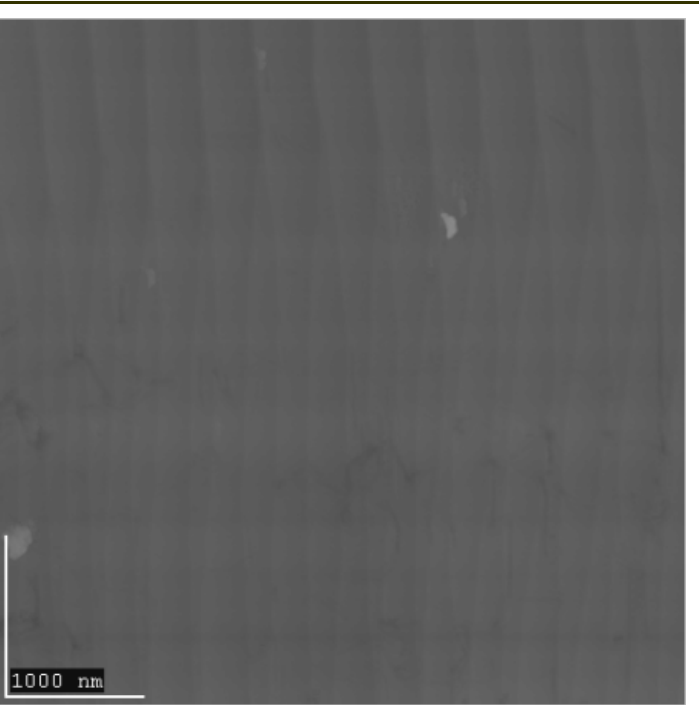

(b)

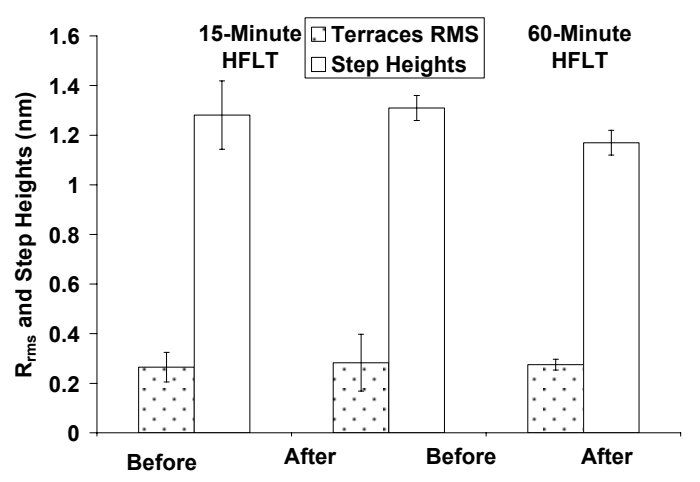

(d)

Figure 4-18. AFM images of the $6 \mathrm{H}-\mathrm{SiC}$ substrate before growth (a) and after 60 minutes of growth using DMS under HFLT conditions (b); FESEM image of the film after 15 minutes of growth using DMS under HFLT conditions (c); Surface roughness and step height data before and after film growth using DMS under HFLT conditions (d). 
were unable to "cure" the atomic steps unveiled by high temperature hydrogen etching of the substrates, nor was it able to create a larger smooth surface as we had hoped.

As seen in Figure 4-18 (c), the step height remained essentially constant before and after growth under HFLT conditions using DMS as source gas. A similar result was observed for growth under LFLT conditions. Examination of cross-sections using FESEM revealed no indication of the film-substrate interface.

\subsection{Heteroepitaxial Growth on Si Substrates}

\subsubsection{High Flux High Temperature Heteroepitaxial MS Growth}

In this set of experiments, films were grown on $\mathrm{Si}$ substrate at $800^{\circ} \mathrm{C}$ front face temperature as measured by the optical pyrometer. The MS flux was $1.9 \mathrm{ML} / \mathrm{sec}$ (high flux). Growth conditions will simply be abbreviated as HFHT (high flux high temperature) in this section. The total growth time was either 15 or 60 minutes. Under high flux (HF) conditions, the total carbon fluence was $1.7 \times 10^{3} \mathrm{ML}$ for 15 minutes and $6.8 \times 10^{3} \mathrm{ML}$ for 60 minutes.

To eliminate the effects of variations in the Auger instrument operations from one growth run to another, the peak-to-peak intensity of the Si-LMM spectra for all pre-growth analyses were referenced back to a single pre-growth Si-LMM scan. The ratio of the reference Si-LMM peak-to-peak intensity to that of a pre-growth Si-LMM scan was calculated, and this ratio was applied to all Si-LMM and C-KLL spectra of the particular growth run.

The AES spectra for the HFHT runs are shown in Figure 4-19. At the completion of 15minute growth run, the Si-LMM spectrum still exhibits a very strong elemental Si component. This can be due to either of three possibilities. First it is possible that the film is continuous but very thin, and the elemental Si component originates from the Si substrate below the film. Second, the film is continuous but the Si may have segregated to the surface by out-diffusion 
through small pinholes along grain boundaries, or through the film itself. (Since SiC is a good diffusion barrier, this requires a very thin film, although possibly thick enough to obscure the substrate peak.) The third possibility is that the film is not continuous (incompletely coalesced), and the elemental signal originates from the uncovered substrate. The likely source of the elemental Si signal will be discussed later with the aid of AFM and FESEM images.

As growth proceeds from 15 to 60 minutes, the Si-LMM line no longer exhibits the

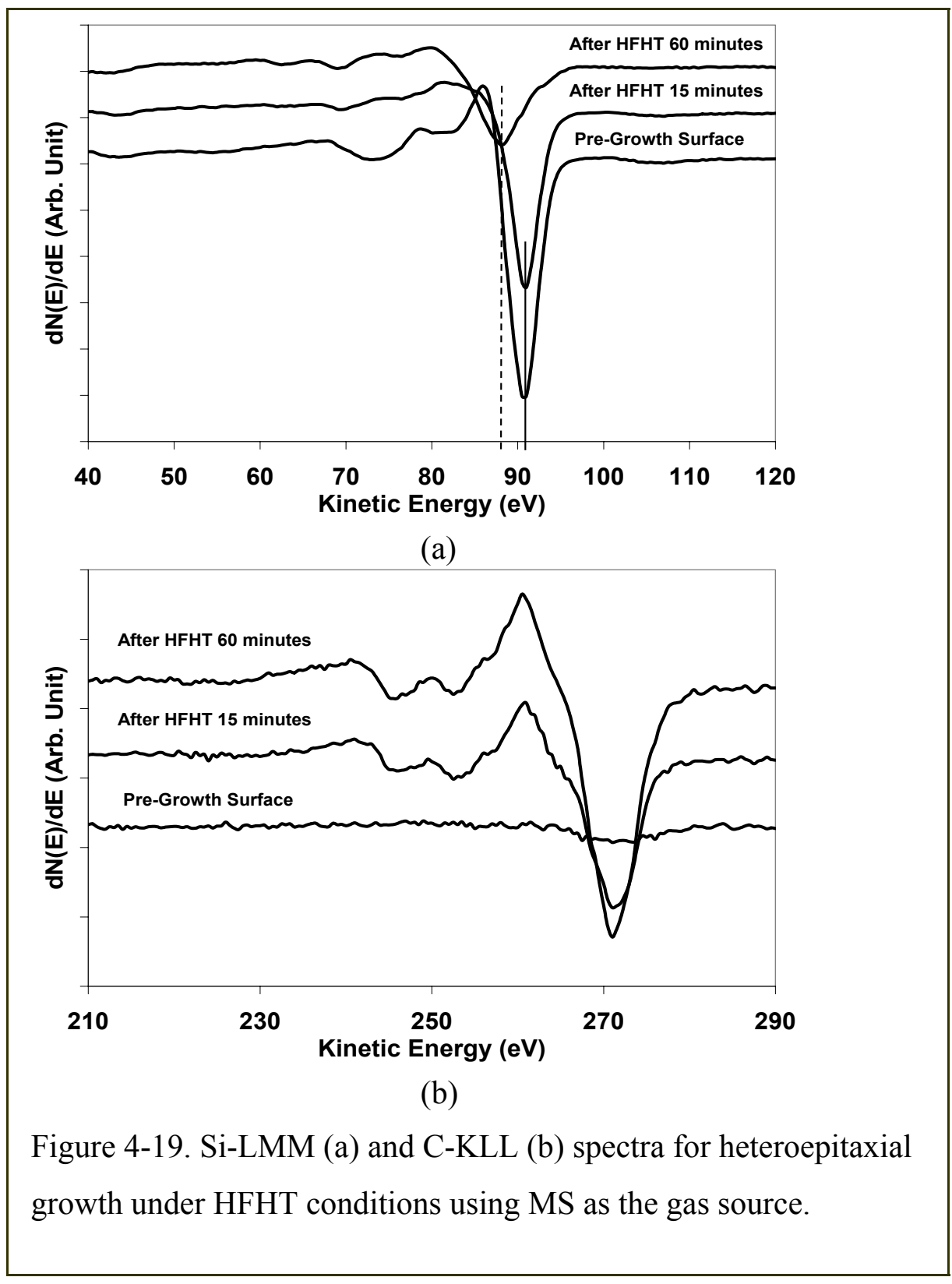




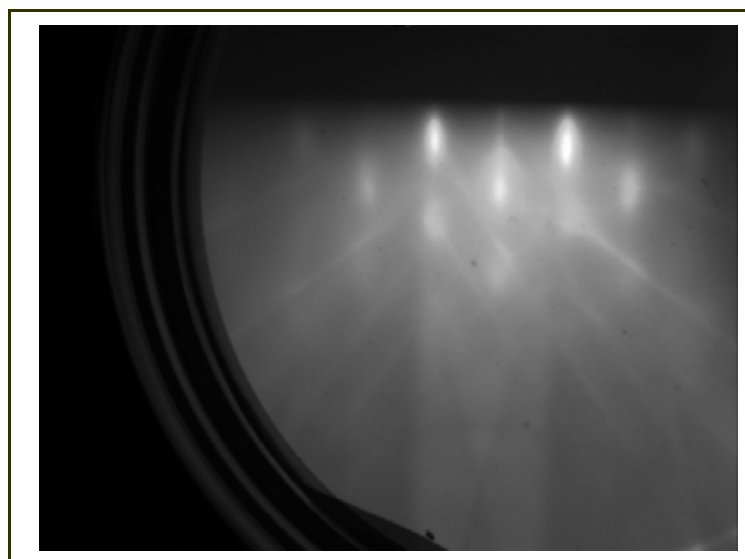

(a)

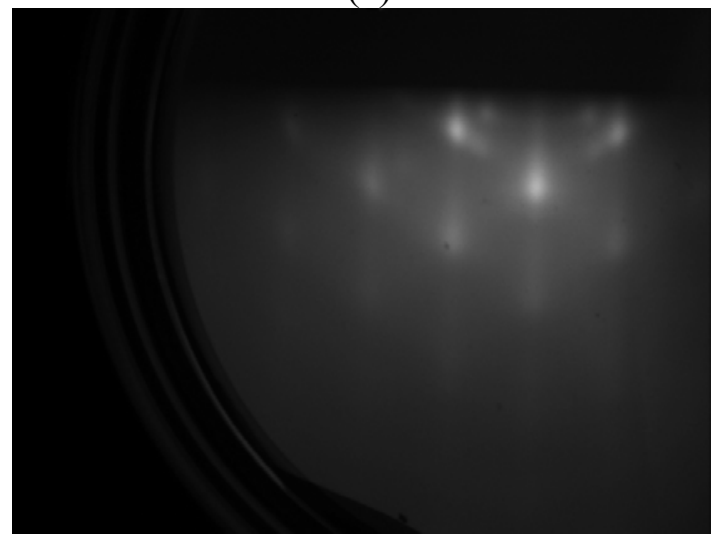

(b)

Figure 4-20. RHEED patterns for $\mathrm{Si}$ substrate before (a) and after (b) 15 minutes of growth using MS under HFHT conditions. elemental component. The peak position had shifted to $87 \mathrm{eV}$, matching the kinetic energy position of SiLMM in SiC. In fact, the 60-minute HFHT AES spectrum is nearly identical to those of the pregrowth $6 \mathrm{H}-\mathrm{SiC}$ substrate. In addition, the peak-topeak intensity of Si-LMM has decreased dramatically, and intensity of C-KLL has increased. For both 15-minute and 60-minute growth runs, the C-KLL line shape were very carbide-like. This is indicative of the case where each $\mathrm{C}$ is bonded to four $\mathrm{Si}$ atoms. There is no evidence for $\mathrm{sp}^{2}$ or $\mathrm{sp}^{3} \mathrm{C}-\mathrm{C}$ bond formation in the C-KLL line shape.

Figure 4-20 shows the RHEED patterns for the Si substrate before growth and after a 15-minute HFHT growth using MS. Note that the RHEED pattern became more spotty after growth. This is a indication of 3-D type of growth on the surface. That is, surface became rougher. The spacing between streaks grew larger corresponding to a decrease in d-spacing of $21 \%$ for both 15 - and 60-minute growth under HFHT conditions using MS. This is in good agreement with the $20 \%$ theoretical lattice parameter mismatch between the substrate $(\mathrm{Si})$ and the film $(3 \mathrm{C}-\mathrm{SiC})$.

The AFM images shown in Figure 4-21 clearly indicate the surface were much rougher after growth than the substrate. Post growth RMS roughness was $0.61 \mathrm{~nm}$ and $2.3 \mathrm{~nm}$ for 15 - 
and 60-mintue growth, respectively. As a comparison, Fenner-etched pre-growth Si substrate has a $\mathrm{R}_{\mathrm{rms}}$ value of between $0.1-0.3 \mathrm{~nm}^{48}$

Figure 21 (c) shows a top view of the sample grown for 60 minutes under HFHT conditions. The image was obtained using a $5 \mathrm{kV}$ electron beam which increased imaging depth. Here voids may be obscured below the film surface, and under close examination, it may be seen that all the voids are "sealed". Figure 4-20 (d) shows the cross sectional view of the film. Here a single void has been isolated. It is clear that the void has been overgrown and sealed by the

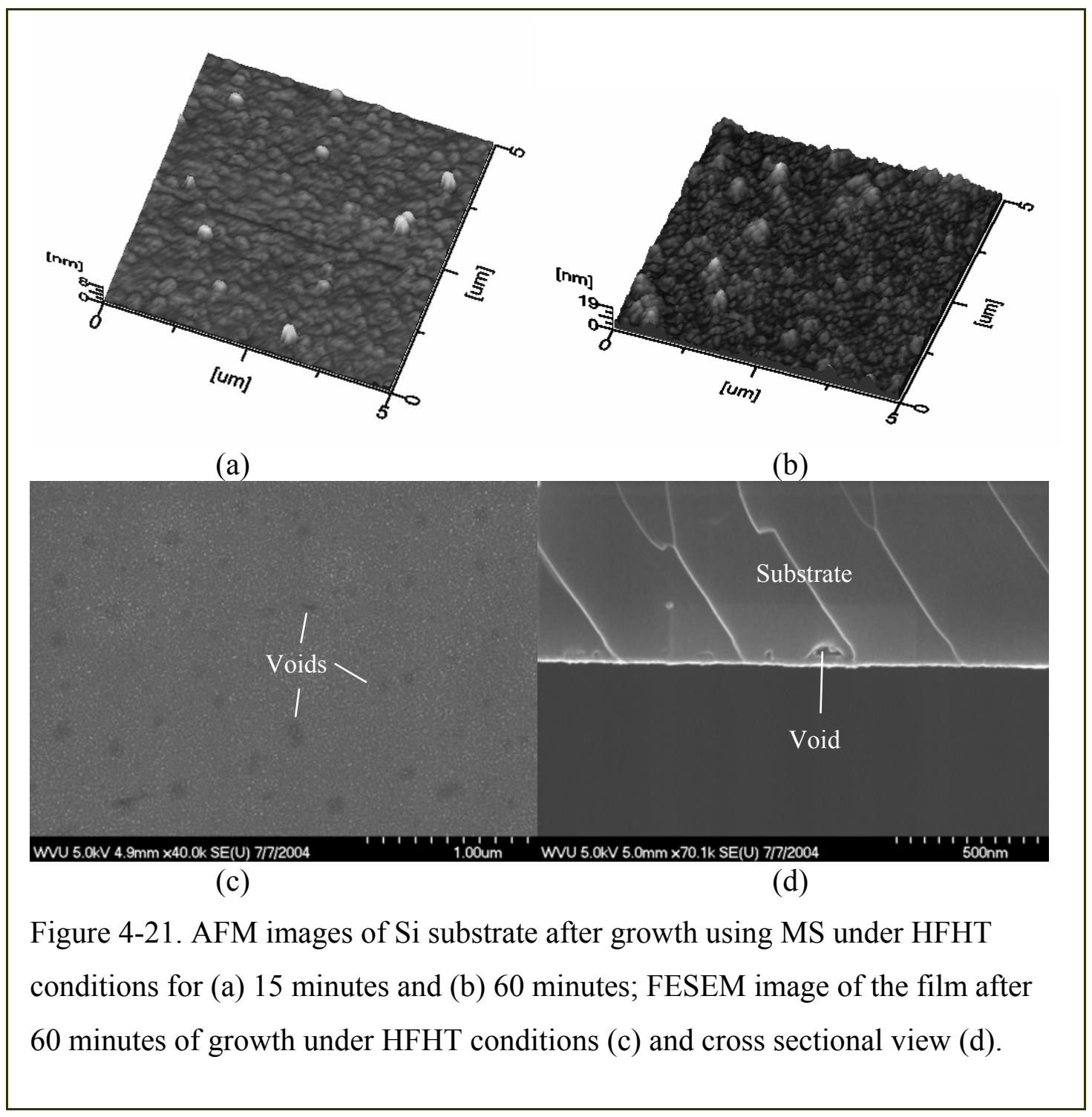


film. This is consistent with the absences of pin holes in the AFM images. Similar observations were made on the HFHT 15-minute sample. The film thickness, based on measurements of thin film covering the voids, was about $19 \mathrm{~nm}$ for the 15 -minute growth run and $46 \mathrm{~nm}$ for the 60 minute growth run.

With this information, we can now answer the question posed earlier about the source of elemental Si component for 15-minute HFHT growth. Si-LMM Auger electrons have a escape depth of about $\sim 2 \mathrm{~nm}$ through $\mathrm{SiC}$ film. Consequently, with a $19 \mathrm{~nm}$ thick, coalesced $\mathrm{SiC}$ film present, the elemental component of the Si-LMM peak could not have originated from the underlying Si substrate. Instead, it must be due to a segregated Si layer on the surface of the SiC film. As mentioned previously, this Si can be supplied by out-diffusion, and, in the presence of added carbon, it can participate in the growth of the $\mathrm{SiC}$ film. ${ }^{48,89,90}$ The Si that produced this segregated layer must in part be provided by the Si lost in forming the voids (growth temperature were too low to evaporate Si). Since the voids were sealed for the $19 \mathrm{~nm}$ thick film, the conclusion is that there were sufficient defects to allow out-diffusion. By the time the film reached $46 \mathrm{~nm}$ thick, these diffusion channels must have been sealed off since the elemental component of the Si-LM peak is no longer present.

\subsubsection{Low Flux High Temperature Heteroepitaxial MS Growth}

In this set of experiments, films were grown on the $\mathrm{Si}$ substrate at $800^{\circ} \mathrm{C}$ front face temperature as measured by the optical pyrometer. The MS flux was $0.2 \mathrm{ML} / \mathrm{sec}$ (low flux). Growth conditions will simply be abbreviated as LFHT (high flux high temperature) in this section. The total growth time was either 15 or 60 minutes. Under low flux (LF) conditions, the total carbon fluence was $0.2 \times 10^{3} \mathrm{ML}$ for 15 minutes and $0.7 \times 10^{3} \mathrm{ML}$ for 60 minutes. 
The AES spectra for LFHT runs using MS as gas source are shown in Figure 4-22. At the completion of the 15-minute growth run, the Si-LMM spectrum was unchanged. Specifically, it still had a perfect elemental Si-LMM line shape. Moreover, the C-KLL peak, though increased in intensity, did not have the line shape of SiC-C. In fact, this particular C-

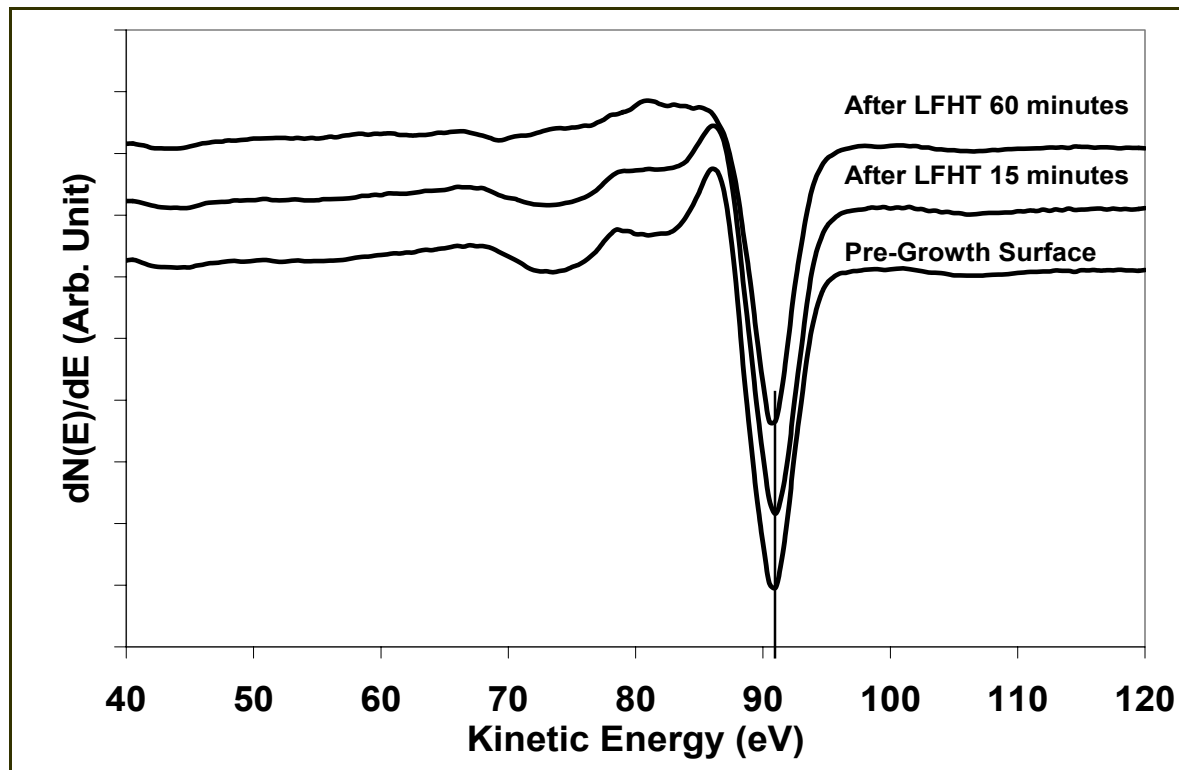

(a)

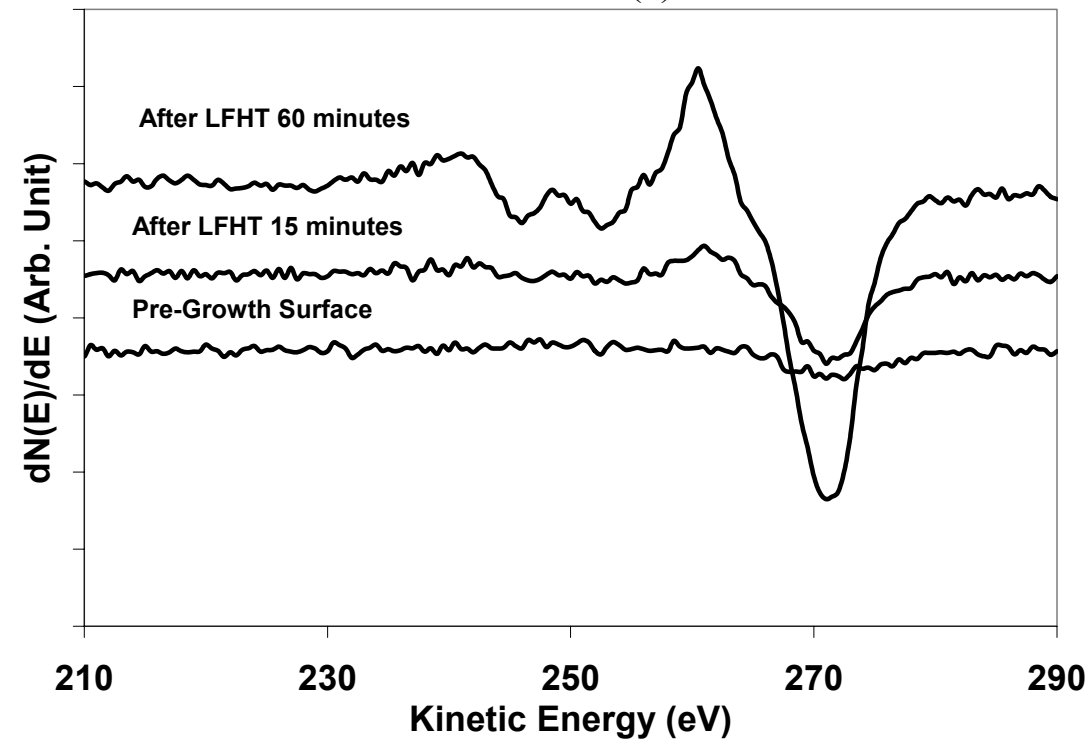

(b)

Figure 4-22. Si-LMM (a) and C-KLL (b) spectra for heteroepitaxial growth under LFHT conditions using MS as the gas source. 
KLL peak did not resemble of any of the three bulk carbon species, $\mathrm{SiC}, \mathrm{sp}^{2}-\mathrm{C}$ (graphite), or $\mathrm{sp}^{3}-$ $\mathrm{C}$ (diamond).

This suggests that although some type of carbon has started to nucleate on the surface, it was not sufficiently developed to display the valence band of any of these bulk species. As growth proceeded, the C-KLL developed a well defined carbide line shape, and the Si-LMM line shape evolved into a convolution of the elemental and SiC-Si. Once again, the Si component of

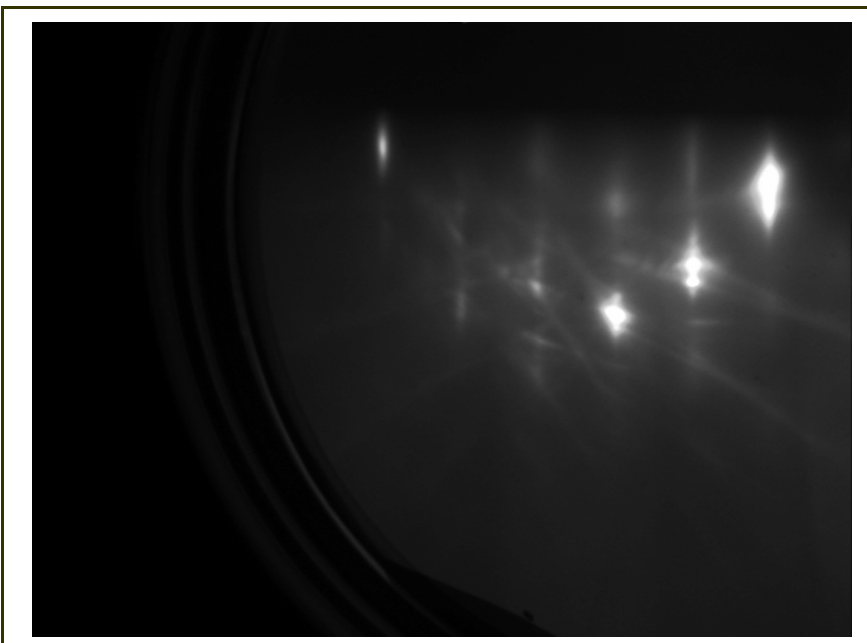

(a)

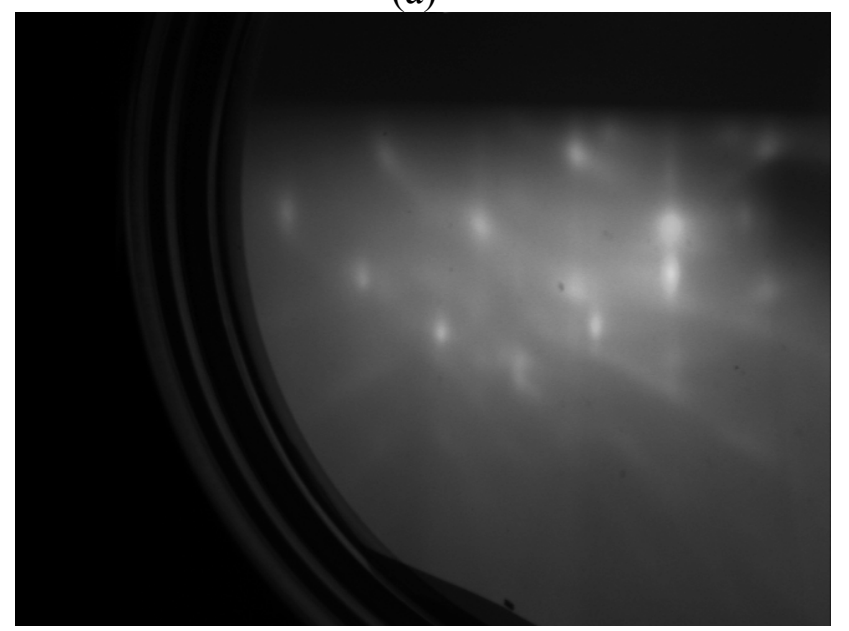

(b)

Figure 4-23. RHEED patterns for $6 \mathrm{H}-\mathrm{SiC}$ substrate before (a) and after (b) 15 minutes of growth using MS under LFHT conditions. the elemental Si-LMM, needs to be determined and may be associated with any of the potential sources described previously. This will be discussed with the aid of the RHEED and AFM data shown below.

Figure 4-23 shows the RHEED patterns of Si substrate before growth and after the 15 minutes of growth under LFHT conditions. The RHEED patterns became more spotty after growth, which was an indication of 3-D type of growth on the surface. As in HFHT growth runs, spacing between streaks increased and corresponding to a reduction in d-spacing drop of $20 \%$ for both the 15 -minute and the 60-minute runs. This corresponds well 
with the change expected for $\beta$-SiC formation. For the 60 -minute growth run, this change is consistent with carbide-like AES spectra earlier. At first look, however, there may seem to be a contradiction between the AES spectra for LFHT conditions after 15 minutes of growth, which showed no carbide characteristics, and the carbide-like RHEED patterns. In actuality, however, this illustrates the fundamental difference between AES and RHEED characterization. Apparently the long range order of the $\beta$-SiC crystal was insufficient to establish bulk-like bond structure, but it was sufficient to establish the bulk-like RHEED pattern.

AFM images of post growth surface for the LFHT growth of 15 and 60 minutes are

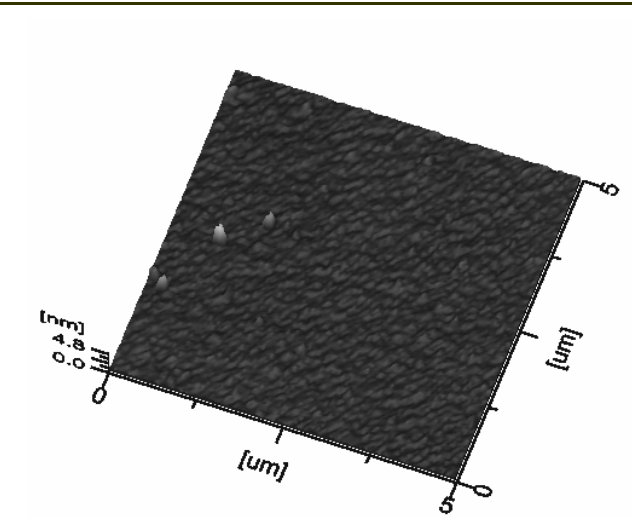

(a)

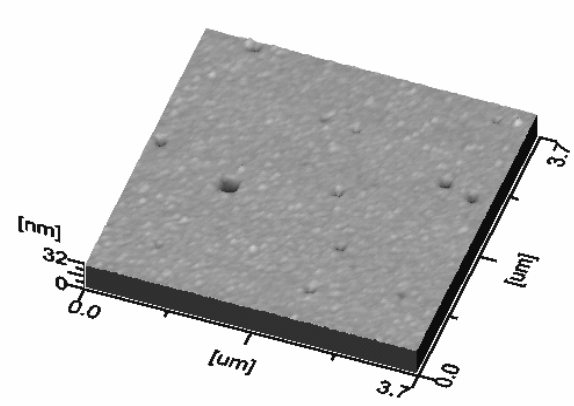

(b)

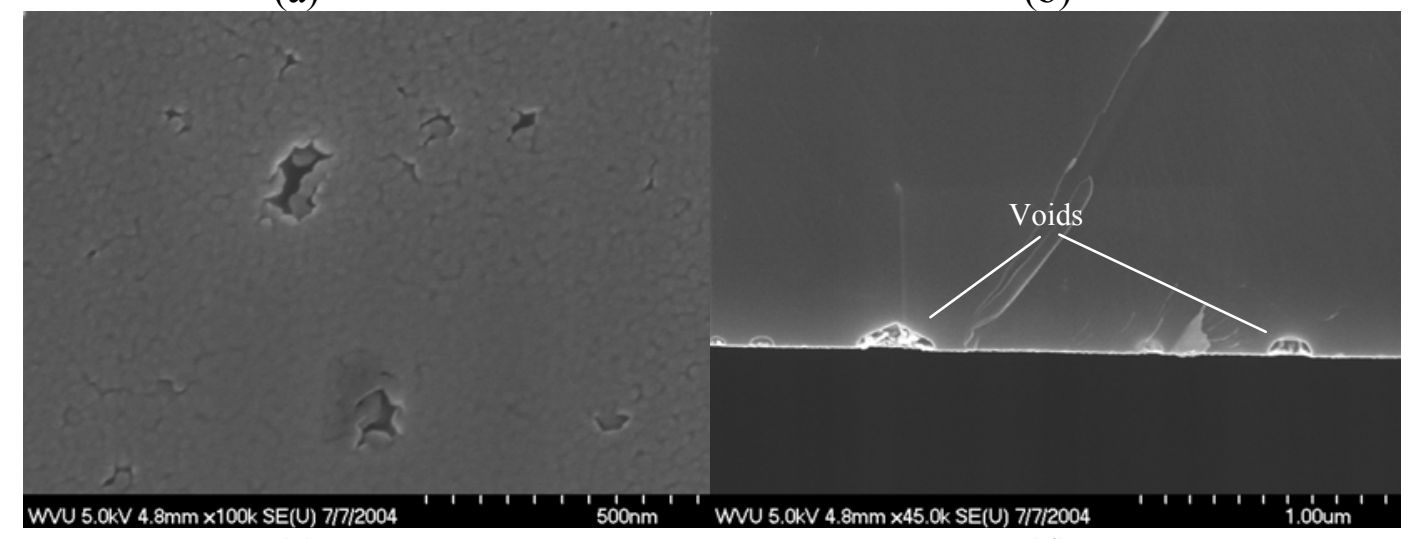

(c)

(d)

Figure 4-24. AFM images of Si substrate after growth using MS under LFHT conditions for (a) 15 minutes and (b) 60 minutes; FESEM image of the film after 60 minutes of growth under LFHT conditions (c) and cross sectional view (d). 
shown in Figure 4-24(a) and (b). Post growth RMS roughness was $0.4 \mathrm{~nm}$ and $1.2 \mathrm{~nm}$ for 15 and 60-mintue growth, respectively. This is much rougher than the initial Si substrate. Large open voids, which could serve as sources of elemental $\mathrm{Si}$, can be seen on the 60-minute LFHT growth sample. This is also evident from the FESEM image in Figure 4-24(c). Because this out-diffusion and segregation of Si to the surface, AES spectra showed elemental Si line shape though the film was already very carbidic as indicated by the C-KLL spectrum and RHEED.

In Figure 4-24 (d), several voids of different sizes can be seen in the cross sectional view of the LFHT 60-minute post-growth sample, same as the AFM data. The open voids, however, prevented proper measurement of actual film thickness. These voids were not sealed by film as in HFHT growth runs, making it impossible to estimate the film thickness from FESEM images. No void or evidence of film/substrate interface was seen on the cross-section of the sample grown under 15-minute LFHT conditions.

\subsubsection{High Temperature Heteroepitaxial DMS Growth}

In this set of experiments, films were grown at $800^{\circ} \mathrm{C}$ front face temperature as measured by the optical pyrometer. The DMS flux was either $0.2 \mathrm{ML} / \mathrm{sec}$ (low flux) or $1.9 \mathrm{ML} / \mathrm{sec}$ (high flux). Growth conditions will simply be abbreviated as HFHT (high flux high temperature) and LFHT (low flux low temperature) in this section. The total growth time was either 15 or 60 minutes. Under low flux (LF) conditions, the total carbon fluence to the samples surfaces was $0.3 \times 10^{3} \mathrm{ML}$ for 15 minutes and $1.3 \times 10^{3} \mathrm{ML}$ for 60 minutes. Under high flux (HF) conditions, the total carbon fluence was $3.4 \times 10^{3} \mathrm{ML}$ for 15 minutes and $13.6 \times 10^{3} \mathrm{ML}$ for 60 minutes.

The AES spectra for the HFHT DMS growth runs are shown in Figure 4-25. The SiLMM peaks for both 15 minutes and 60 minutes of growth are carbide-like with little if any elemental Si component. Interestingly, however, they have not shifted to the position $(87 \mathrm{eV})$ 


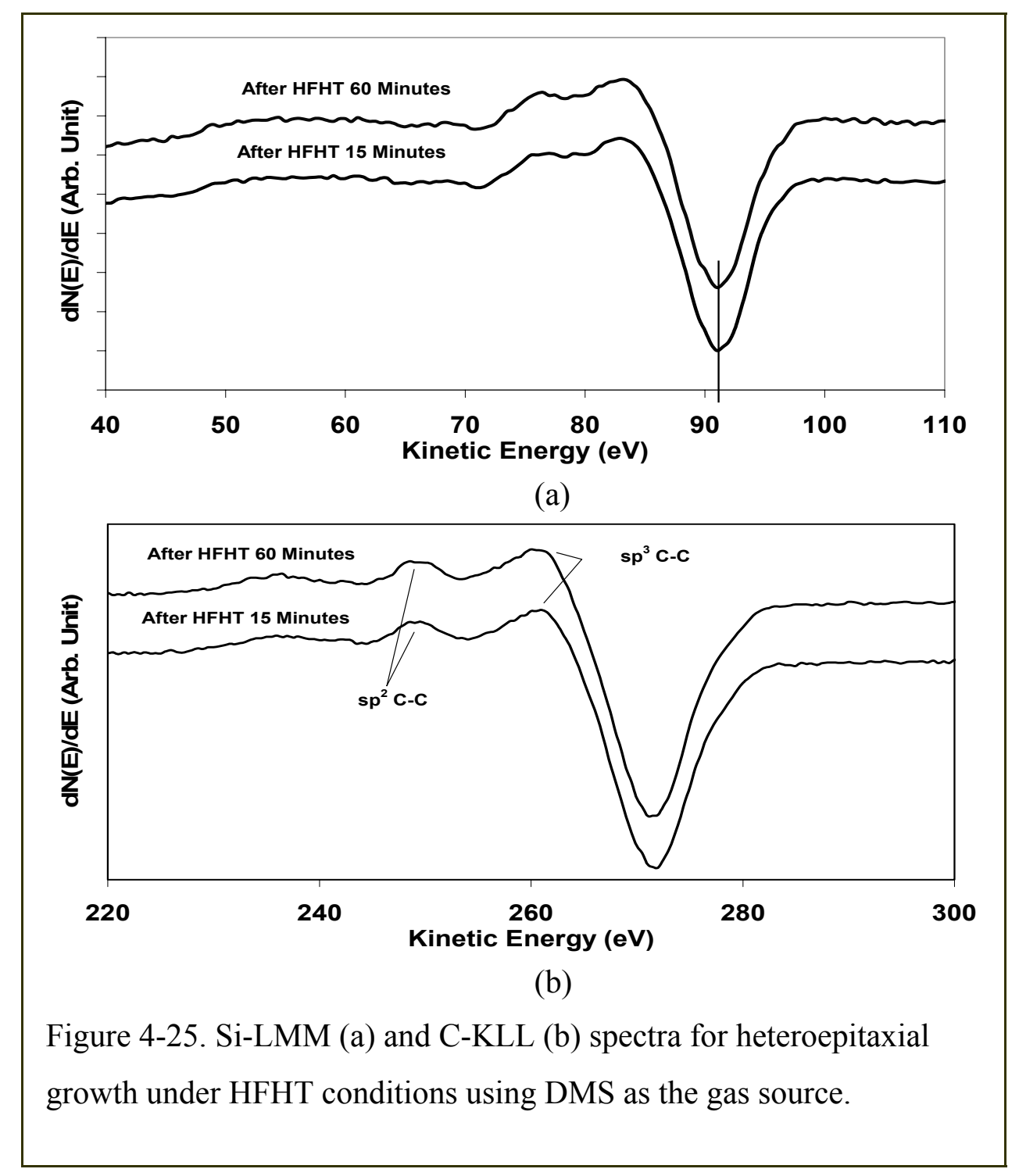

expected for $\mathrm{SiC}$ based on the bulk $\mathrm{SiC}$ standard and the HFHT film discussed earlier. The CKLL spectra exhibit contributions from $\mathrm{sp}^{2}$ and $\mathrm{sp}^{3} \mathrm{C}-\mathrm{C}$ species. This is not surprising since the C-Si ratio for DMS is two. It appears that every Si-atom in the film is coordinated with $4 \mathrm{C}$ atoms but at lease some carbon atoms are coordinated with one or more carbon atoms for the HFHT growth conditions using DMS.

The FESEM images for the 60 minute HFHT sample are shown in Figure 4-26. For this sample, no voids were observed, but based on the fracture pattern, the film appears to be quite 


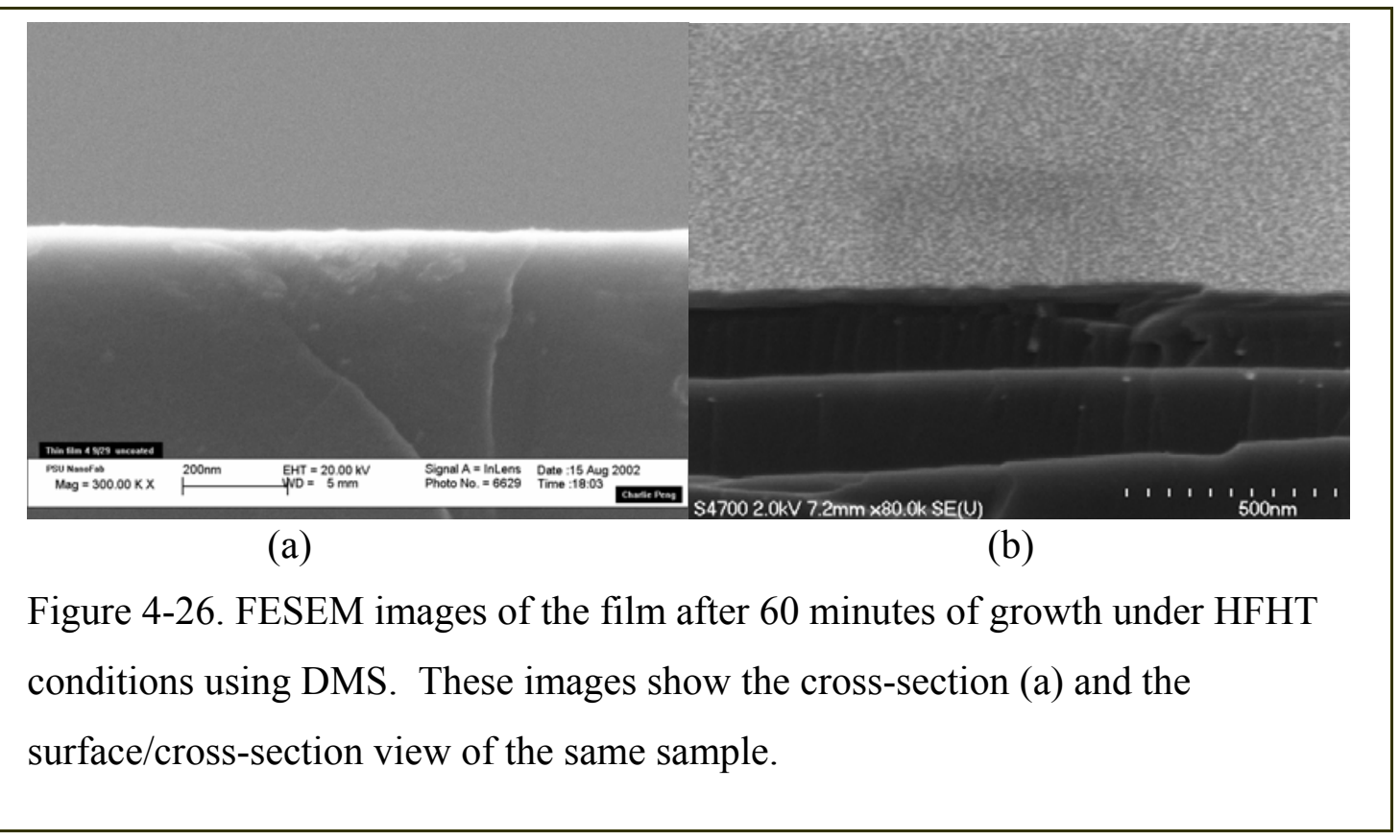

thick. The best estimate is that the film is $\sim 32 \mathrm{~nm}$ thick for the 60 -minute growth under HFHT conditions. The situation is essentially the same for the 15-minute growth at HFHT conditions except that the film is $\sim 10 \mathrm{~nm}$ thick. It appears that under the HFHT conditions, $\mathrm{SiC}$ growth was sufficiently rapid to stop Si out-diffusion before pits could form.

The Si-LMM and C-KLL spectra of the LFHT growth runs are displayed in Figure 4-27. Here the C-KLL spectra are clearly very carbide-like, but now the Si-LM spectra are a mixture of SiC-Si and elemental Si.

The FESEM images for the 60-minute growth run under LFHT conditions are shown in Figure 4-28. Here the top view and the cross section both show many unsealed voids, and from the cross section view, the thickness of the film is observed to be $19 \mathrm{~nm}$. This film was fully coalesced with the exception of the opening at the voids, and it was too thick for the elemental component of the Si-LMM peak to originate from the underlying Si substrate. In this case, the Si must be segregated onto the surface of the SiC film. Apparently, under the LFHT conditions, the growth was not sufficiently rapid to establish a good diffusion barrier. As a result, Si 


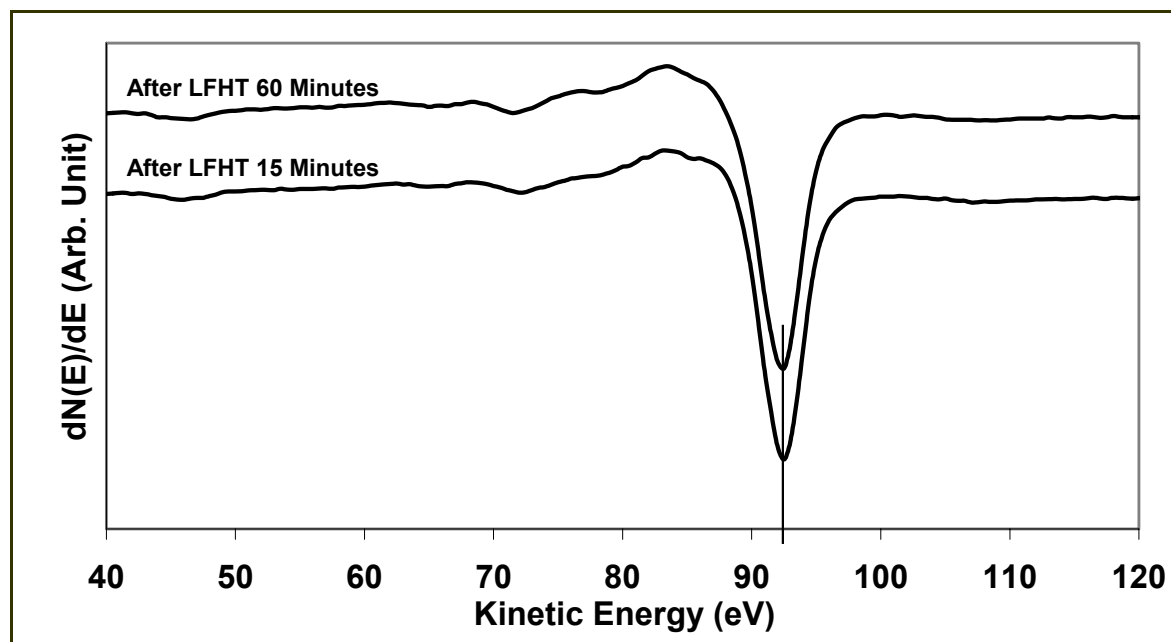

(a)

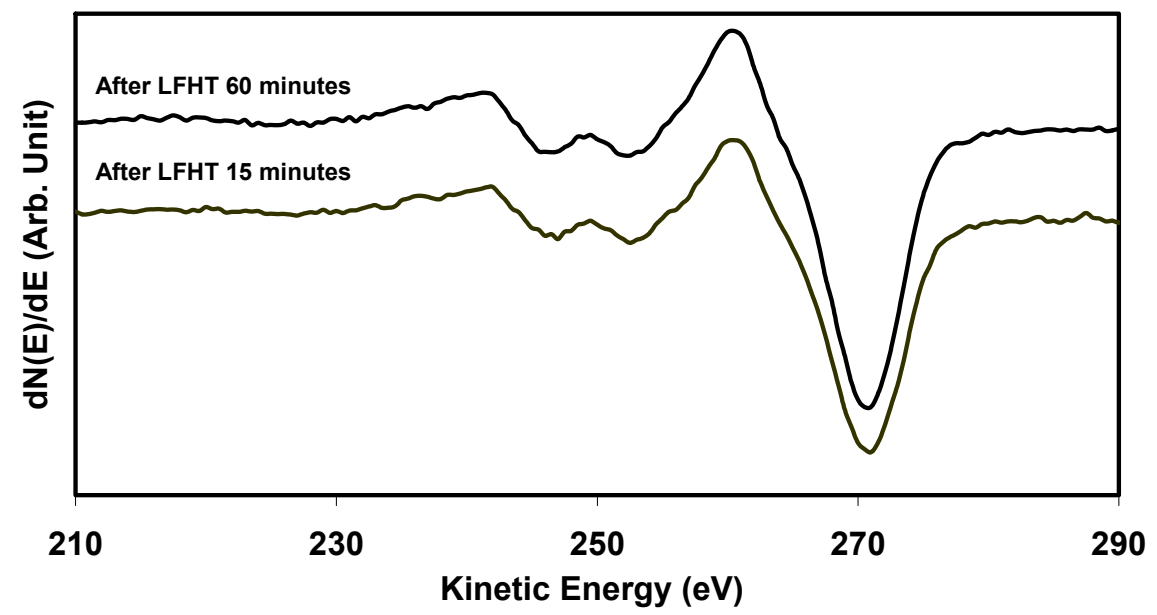

(b)

Figure 4-27. Differentiated Auger Si-LMM (a) and C-KLL (b) peak line shapes comparison at $800^{\circ} \mathrm{C}$ for low flux runs.

continued to out-diffuse and react with DMS to form SiC. Under these conditions, the voids will continue to grow until the SiC film is sufficiently thick to stop the diffusion process. Until that point is reached, the Si-LMM will continue to have both SC-Si and elemental Si components and the C-KLL will be carbide like. After that, the Si-LMM will become carbide-like and the CKLL will have $\mathrm{sp}^{2}$ and $\mathrm{sp}^{3}$ components.

This is distinctly different from the HFHT runs using DMS as gas source. As stated above, for HFTH the diffusion barrier is set up very quickly. Si from the substrate did not 


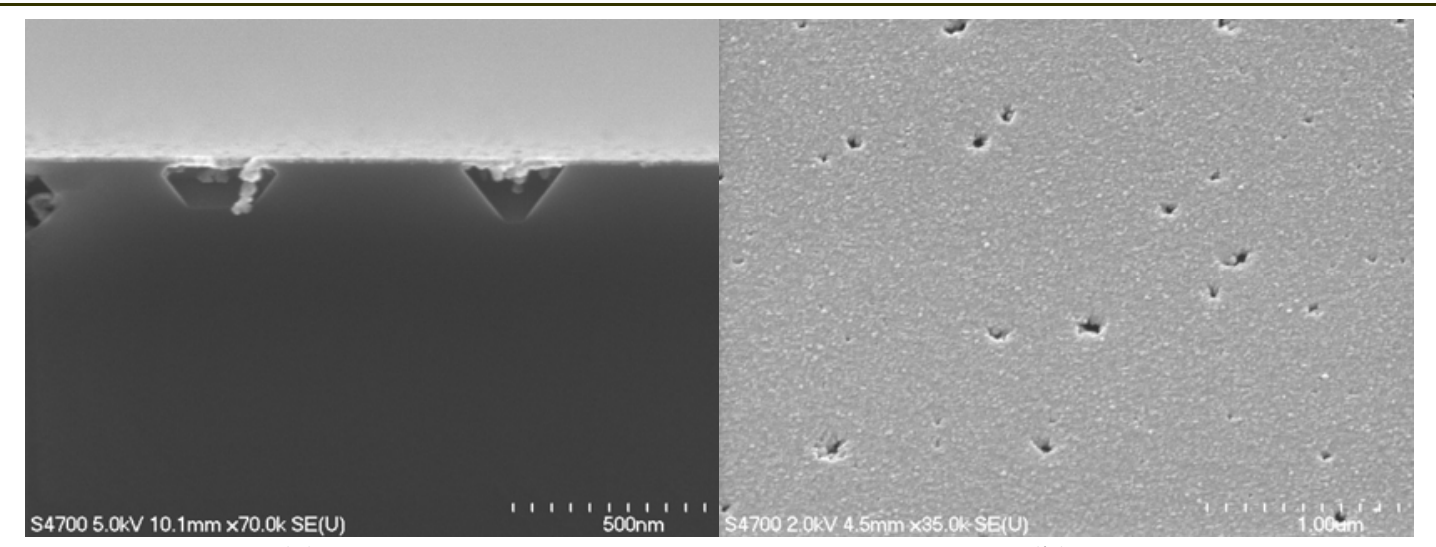

(a)

(b)

Figure 4-28. FESEM images of the film after 60 minutes of growth under LFHT conditions using DMS. There images show the cross-section (a) and the surface (b) view of the same sample.

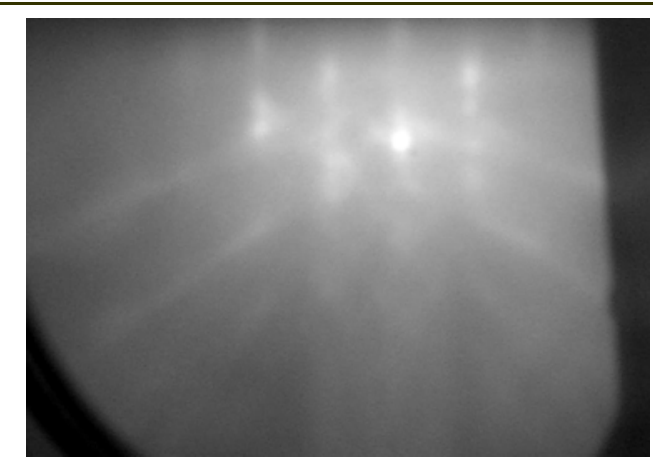

(a)

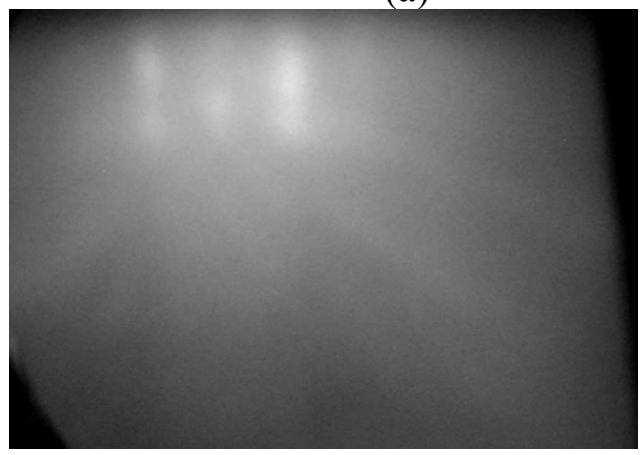

(b)

Figure 4-29. RHEED patterns for the Si substrate before (a) and after (b) 15 minutes of growth with DMS under LFHT conditions.. participate in the growth process. Consequently no voids are formed. From the onset, growth took place under carbon rich conditions, and so the Si-LMM was carbide-like and the C-KLL had both $\mathrm{sp}^{2}$ and $\mathrm{sp}^{3}$ components.

Figure 4-28 shows the RHEED patterns of Si substrate before and after a 15-minute LFHT growth. The spacing between streaks grew larger after the growth run, and corresponded to an $8 \%$ decrease in the $\mathrm{d}$-spacing, less 
than the theoretical lattice parameter of $20 \%$ between $\mathrm{Si}$ and $3 \mathrm{C}-\mathrm{SiC}$. We believe this is representative of the $\mathrm{Si}$ ad-layer on the $\mathrm{SiC}$ surface.

\subsubsection{Low Temperature Heteroepitaxial DMS Growth}

In this set of experiments, films were grown on $\mathrm{Si}$ substrate at $700^{\circ} \mathrm{C}$ front face temperature as measured by the optical pyrometer. Growth conditions will again be abbreviated as HFHT, LFHT, etc., in this section. The total growth time was either 15 or 60 minutes. Under DMS high flux (HF) conditions, the total carbon fluence was $3.4 \times 10^{3}$ ML for 15 minutes and $13.6 \times 10^{3} \mathrm{ML}$ for 60 minutes. Under DMS low flux (LF) conditions, the total carbon fluence to the samples surfaces was $0.4 \times 10^{3} \mathrm{ML}$ for 15 minutes and $1.4 \times 10^{3} \mathrm{ML}$ for 60 minutes.

The AES spectra for the LFLT DMS growth runs are shown in Figure 4-30. The SiLMM spectrum for the 15-minute growth clearly displays an elemental shoulder along with some $\mathrm{SiC}$ characteristics. After 60 minutes of growth, the shoulder is less obvious and the spectrum is more carbide-like. The line shape for 15-minute C-KLL showed that there was a mixture of $\mathrm{sp}^{2}$ and carbide bonds at that point. This was expected due to the extra methyl group in the source gas - DMS.

The FESEM for the low temperature runs revealed no voids, and the fracture patterns ran up to the surface of the substrate. Consequently, we believe the film is very thin, thus it is quite possible that the Si-LMM Auger electrons from the substrate were able to penetrate through film, that is, the film thickness was less than the AES sampling depth. This is consistent with the fact that the C-KLL spectra have significant $\mathrm{sp}^{2}$ and $\mathrm{sp}^{3}$ character which does not occur in the presence of a segregated Si ad-layer. 


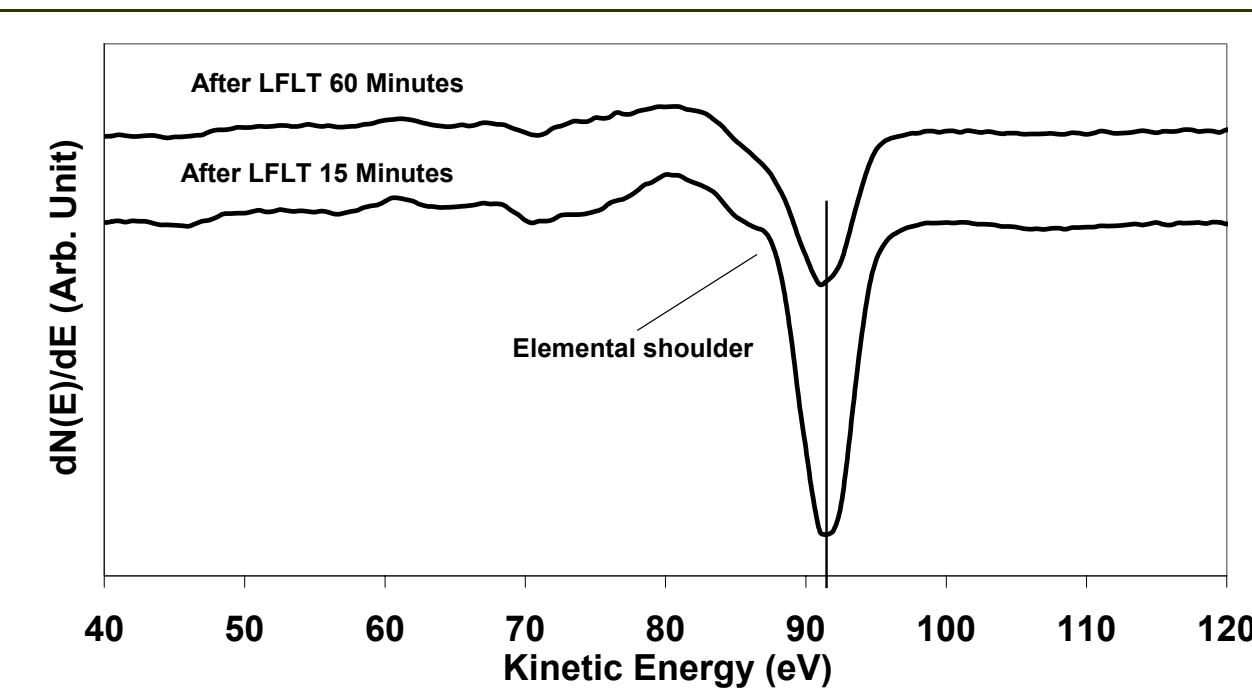

(a)

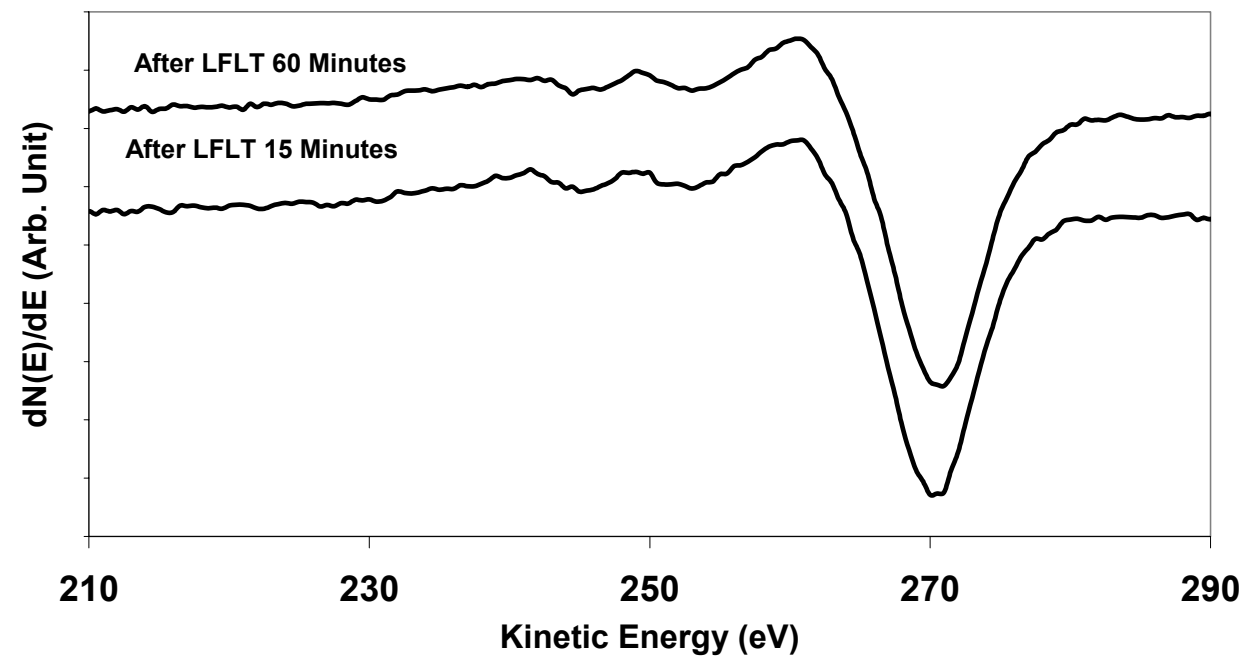

(b)

Figure 4-30. Si-LMM (a) and C-KLL (b) spectra for heteroepitaxial growth under LFLT conditions using DMS as the gas source.

The high flux growth spectra are shown in Figure 4-31. There was almost no elemental Si shoulder for the 15-minute growth and no evidence of elemental should for the 60-minute growth. As the growth time increased from 15 to 60 minutes under HFLT conditions, the film had grown thicker enough to attenuate the elemental Si Auger electrons originating from the substrate. In support of this, the Si-LMM peak-to-peak intensity decreased slightly. The C-KLL spectra exhibited significant $\mathrm{sp}^{2}$ and $\mathrm{sp}^{3}$ character. 


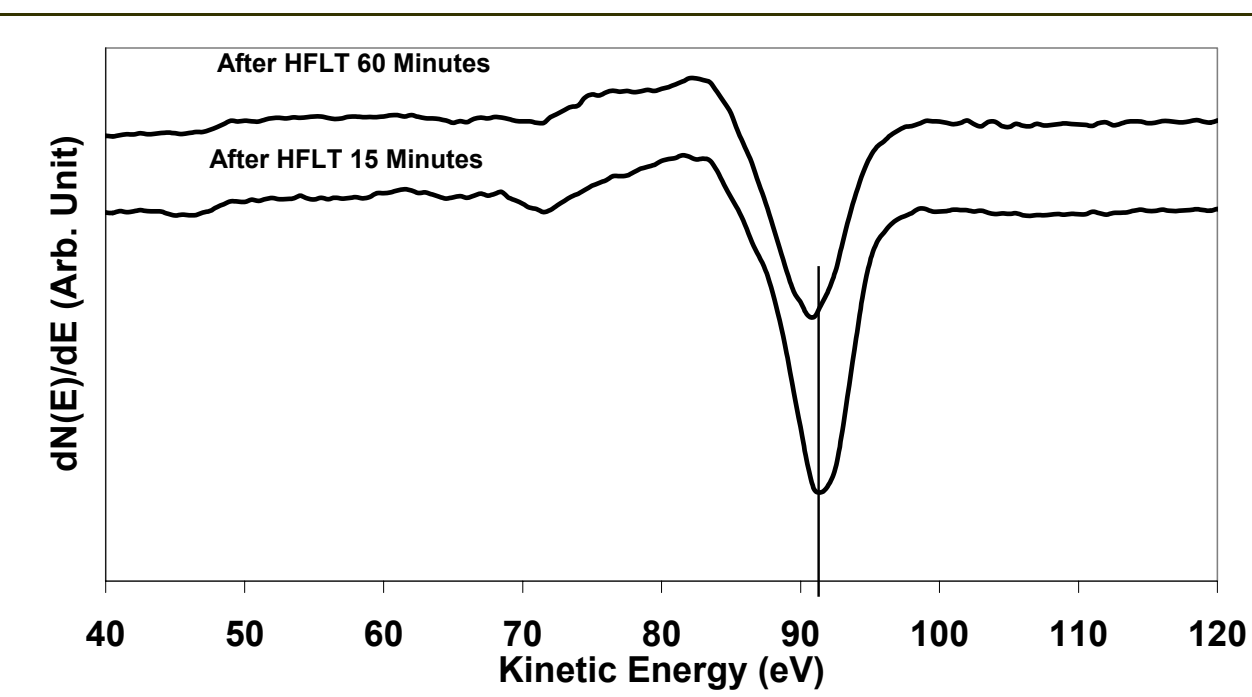

(a)

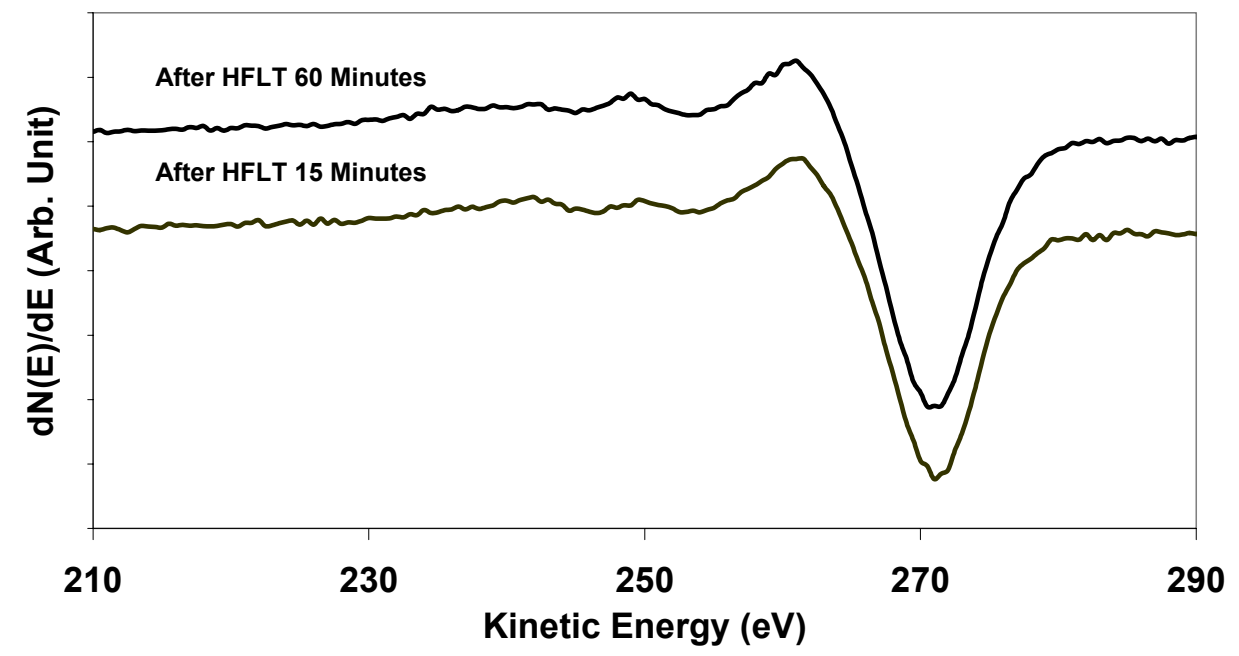

(b)

Figure 4-31. Si-LMM (a) and C-KLL (b) spectra for heteroepitaxial growth under HFLT conditions using DMS.

As stated earlier, FESEM images taken on the cross-section of the LFLT and HFLT samples did not reveal any voids or usable fracture patterns. The RHEED images also did not show any distinguishable differences before and after growth. 


\section{Chapter 5: CONCLUSIONS AND}

\section{RECOMMENDATIONS}

\subsection{Conclusions of Results}

\subsubsection{High Temperature Hydrogen Etching}

Using high temperature hydrogen etching, we have demonstrated the ability to produce periodically stepped $6 \mathrm{H}-\mathrm{SiC}$ substrates consistently. The typical terrace widths were $\sim 200 \mathrm{~nm}$ while step heights were $\sim 1.3 \mathrm{~nm}$ for an on-axis surface. For $3.5^{\circ}$ off-axis surfaces, step heights were $\sim 1.5 \mathrm{~nm}$ and terrace widths were $\sim 30 \mathrm{~nm}$. In both cases, the step height was nominally one unit cell or $6 \mathrm{Si}-\mathrm{C}$ bilayers.

Many experimental variables influence the etch rate and the quality of the etched surfaces. Those studied here included substrate temperature, dwell time, gas composition, and gas flow rate. The optimum parameters for our setup were $1600^{\circ} \mathrm{C}$ substrate temperature, no dwell time, $8 \mathrm{SCFH}(8.0 \mathrm{~cm} / \mathrm{s}$ linear velocity), and a gas composition of $5 \%$ hydrogen in argon. This set of optimum experimental parameters was used to produce all the $6 \mathrm{H}-\mathrm{SiC}$ substrates for the ensuing thin film growth studies. The process could be scaled up to handle large $\mathrm{SiC}$ wafers.

HF acid-based wet chemical surface pre-treatment methods were unable to create a hydrogen-terminated, oxide-free surface on $6 \mathrm{H}-\mathrm{SiC}$ substrates comparable to the Fenner-etched silicon substrate. An oxide was present at low levels on the surface of even our best $6 \mathrm{H}-\mathrm{SiC}$ surfaces. It is not clear whether the oxide was a result of bulk-growth during the etch process or contacting air as the sample was transported. 


\subsubsection{Epitaxial Growth of SiC}

For homoepitaxial growth on the 6H-SiC (0001) Si-face using MS and DMS, little or no change in surface chemical composition, crystal structure, or RMS roughness was observed. Decreases of step height from nominally $6 \mathrm{SiC}$ bilayers to 3 bilayers were observed and are consistent with the other observations of step flow growth on $6 \mathrm{H}-\mathrm{SiC}$ using chemical vapor deposition. Oxide on the surface of the substrate very likely played a role in limiting the homoepitaxial film growth. The carbon-rich gas source, DMS, did not initiate the growth of $\mathrm{sp}^{2}$ or $\mathrm{sp}^{3}$ hybridized C-C species as observed in heteroepitaxial growth on Si substrates. This may be due to formation of volatile species such as $\mathrm{CO}$ or $\mathrm{CH}_{\mathrm{x}} \mathrm{O}_{\mathrm{y}}$ by surface reactions between the excess carbon adspecies and the surface oxide.

For heteroepitaxial growth on the H-terminated $\mathrm{Si}(001)$ surface using $\mathrm{DMS}$ at $800^{\circ} \mathrm{C}$, our results showed that the growth process relied on both direct growth from the $\mathrm{Si}-\mathrm{C}$ species in DMS and on segregation and out-diffusion of Si from the substrate. Specifically, the segregated Si reacted with the excess carbon in the DMS molecule. Films previously grown with TMS were examined as a part of this study and they appear to grow by the same process. The out-diffusing silicon leaves voids at the substrate/film interface under high temperature $\left(800^{\circ} \mathrm{C}\right)$ growth conditions for both DMS and TMS sources gases. When the film is sufficiently thick to stop the Si out-diffusion, the excess carbon subsequently forms C-C species in addition to silicon carbide. For growth temperatures of $700^{\circ} \mathrm{C}$, Si has much less mobility and only very thin films are formed. In these cases, the C-KLL spectra clearly indicate the formation of C-C bonds.

MS, which has a one to one $\mathrm{Si} / \mathrm{C}$ molecular ratio, proved to be an excellent gas source for growing thicker carbide films without the formation of $\mathrm{C}-\mathrm{C}$ bonds. However, the current 
experimental parameters were not sufficient to create an effective diffusion barrier and many voids appeared. This problem should be eliminated by working at higher MS fluxes.

DMS growth under LFLT conditions is a good method for producing very thin $(\sim 1 \mathrm{~nm}$ thick) films on Si. For these conditions, the growth process seems to be self-limiting. This is possibly due to the very high stability of the C-C bonds terminating the surface.

\subsection{Significance of the Work}

This research project was funded partially by three different agencies: DARPA, ONR, DOE, and NASA, each with separate objectives. The study has completely or partially fulfilled these objectives, they include: the creation of a smoother, periodically stepped surface on $6 \mathrm{H}-$ $\mathrm{SiC}$ substrates using $\mathrm{H}$-etching, the development of a suitable $\beta-\mathrm{SiC} / \mathrm{Si}$ substrates for epitaxial growth of other advanced semiconductors such as $\mathrm{GaN}$, and the development of SiC-based sensors and electronics to be used in harsh environments.

To our knowledge, this work is the first to report observations of step-bunching during thin film growth under gas source molecular beam epitaxy conditions. Moreover, the growth of $\beta$-SiC at $800^{\circ} \mathrm{C}$ using MS, as demonstrated by RHEED and AES, appears to be the lowest reported epitaxial growth temperature. Finally, we are the first to report the self-limiting nature of the growth process at $700^{\circ} \mathrm{C}$.

\subsection{Recommendations}

The origin of oxide and formation mechanism on the surface of $6 \mathrm{H}-\mathrm{SiC}$ should be clarified. An in-situ ultra high vacuum etching method should be developed to remove the residual oxide prior to further processing of the $6 \mathrm{H}-\mathrm{SiC}$. The limited growth of homoepitaxial films on $6 \mathrm{H}-\mathrm{SiC}$ using either MS or DMS under the current growth parameters suggests the need 
to study higher growth temperatures and/or higher gas fluxes in addition to the use of oxide free surfaces.

Voids still occur at the film/substrates interface during heteroepitaxial growth using MS as the gas sources. Other experiments using TMS and DMS proved that with sufficient carbon flux, diffusion barriers thick enough to prevent Si out-diffusion can be formed. Increasing the MS gas fluxes may prompt the creation of such a barrier during the initial stages of growth at $800^{\circ} \mathrm{C}$, thus producing a void-free silicon carbide film on the substrate. 


\section{References}

${ }^{1}$ P.G. Neudek, R.S. Okojie, L.Y. Chen, "High-Temperature Electronics-A Role for Wide Bandgap Semiconductors?", Proceedings of the IEEE, Vol. 90, No. 6, June 2002.

${ }^{2}$ D.K. Ferry, "High-Field Transport in Wide-Band-Gap Semiconductors", Physics Review B, Vol. 12, 2361 (1975).

${ }^{3}$ R.S. Kern, R.F. Davis, "Deposition and Doping of Silicon Carbide by Gas-Source Molecular Beam Epitaxy," Applied Physics Letters, Vol. 71, No. 10, 1356 (1997).

${ }^{4}$ G.R. Fisher, P. Barnes, "Towards a Unified View of Polytypism in Silicon Carbide," Philosophical Magazine B, Vol 61, No. 2, 217 (1990).

${ }^{5}$ Majority of data here taken from Office of Naval Research website, numbers are up-to-date as June 21, 2004, www.onr.navy.mil/sci_tech/information/312_electronics/ncsr/materials/sic.asp

6 M. Mehregany, C. Zorman, N. Rajan, C. Wu, "Silicon Carbide MEMS for Harsh Environment," Proceedings of the IEEE, Vol. 86, No. 8, 1594(1998).

${ }^{7}$ N.G. Hingorani, K.E. Stahlkopf, "High-Power Electronics," Scientific American, Vol. 269, 78 (1993).

${ }^{8}$ R.J. Trew, J. Yan, P.M. Mock, "The Potential of Diamond and SiC Electronic Devices for Microwave and Millimeter-Wave Power Applications," Proceedings of the IEEE, Vol. 79, 598, 1991.

9 S.J. Przybulko, "Developments in Silicon Carbide for Aircraft Porpulsion System Applications," American Institute of Aeronautics and Astronautics, Washington, DC, Report June 1993.

10 W.C. Nieberding and J.A. Powell, "High Temperature Electronic Requirements in Aeropropulsion Systems," IEEE Transactions on Industrial Electronics, vol. 29, 103 (1982)

${ }^{11}$ M. Bhatnagar, B.J. Baliga, "Comparison of 6H-SiC, 3C-SiC, and Si for Power Devices," IEEE Transactions on Electron Devices, Vol. 40, 1112, 1994.

${ }^{12}$ R.F. Davis, "III-V Nitrides for Electronic and Optoelectronic Applications", Proceedings of the IEEE, Vol. 79, 702 (1991).

13 J.H. Edgar, "Prospects for Device Implementation of Wide Band Gap semiconductors", Journal of Materials Research, Vol. 7, No. 1, 235 (1992).

${ }^{14}$ Y.M. Tairov, V.F. Tsvetkov, 1981. "General Principles of Growing Large-Size Single Crystals of Various Silicon Carbide Polytypes," Journal of Crystal Growth, Vol. 52, 146 (1981). 
${ }^{15}$ A.G. Acheson, English Pattern, No. 17911, (1892).

${ }^{16}$ J.A. Lely, Berichte der Deutschen Keramischen Gesellschaft e.V, 32, 229, (1955)

${ }^{17}$ D.L. Barrett, J. P. McHugh, H. M. Hobgood, R. H. Hopkins, P. G. McMillin, R.C. Clark, W. J. Choyke, "Growth of Large SiC Single Crystal," Journal of Crystal Growth. Vol. 128, 356 (1993).

18 D.L. Barrett, R. G. Seidensticker, W. Gaida, R. H. Hopkins and W. J. Choyke, "SiC Boule Growth by Sublimation Vapor Transport," Journal of Crystal Growth, Vol. 109, Issue 1-4, 17 (1991).

19 S. Rendakova, V. Dmitriev, "Micropipe Overgrowth and Dislocation Density Reduction in Commercial 6H-SiC and 4H-SiC Wafers," Fall '97 MRS Meeting, December, 149 (1997).

${ }^{20}$ F.C. Frank, "Capillary Equilibria of Dislocated Crystals”, Acta Crystallographica, Vol. 4, 497 (1951).

${ }^{21}$ P.G. Neudeck, W. Huang, M. Dudley, "Breakdown Degradation Associated with Elementary Screw Dislocations in 4H-SiC p+n Junction Rectifiers", Materials Research Society Symposium - Proceedings, Vol. 483, 285 (1997).

${ }^{22}$ H. Iwata, U. Lindefelt, S. Oberg, Sven; P. Briddon, "Theoretical Study of Planar Defects in Silicon Carbide", Journal of Physics: Condensed Matter, Vol. 14, No. 48, 12733, (2002).

${ }^{23}$ R.C. Glass, L.O. Kjellberg, V.F. Tsvetkov, J.E. Sundgren, E. Janzen, "Structural macrodefects in 6H-SiC wafers", Journal of Crystal Growth, Vol. 132, No. 3-4, 504 (1993).

${ }^{24}$ M. Tuominen, R. Yakimova, R.C. Glass, T. Tuomi, E. Janzen, "Crystalline imperfections in 4H SiC grown with a seeded Lely method”, Journal of Crystal Growth, Vol. 144, No. 3-4, 267 (1994).

${ }^{25}$ L. Larson, CREE SiC Substrate Pricing Guide, August 2003.

${ }^{26}$ S. Nishino, J.A. Powell, H.A. Will, "Production of Large-Area Single-Crystal Wafers of Cubic SiC for Semiconductor Devices," Applied Physics Letters, Vol. 42, No. 5, 460 (1983).

${ }^{27}$ M. Mehregany, C. Zorman, "SiC MEMS: Opportunities and Challenge for Applications in Harsh Environments," Thin Solid Films, Vol. 355, 518 (1999).

${ }^{28}$ H. Nakazawa, M. Suemitsu, S. Asami, "Formation of High Quality SiC on Si (100) at $900^{\circ} \mathrm{C}$ using Monomethylsilane Gas-source MBE," Materials Science Forun, Vol. 338-342, 269 (2000).

${ }^{29}$ I.H. Khan, R.N. Summergrad, "The Growth of Single-Crystal Films of Cubic Silicon Carbide on Silicon," Applied Physics Letters, Vol. 11, No. 1, 12 (1967). 
${ }^{30}$ C.J. Mogab, H.J. Leamy, "Conversion of Si to Epitaxial SiC by Reaction with $\mathrm{C}_{2} \mathrm{H}_{2}$," Journal of Applied Physics, Vol. 45, No. 3, 1075 (1974).

31 S. Nishino, Y. Hazuki, H. Matsunami, T. Tanaka, "Chemical Vapor Deposition of Single Crystalline $\beta$-SiC Films on Silicon Substrate with Sputtered SiC Intermediate Layer, ” Journal of the Electrochemical Society, Vol. 127, No.12, 2674 (1980).

${ }^{32}$ H. Ono, Master's thesis, Kyoto University, Kyoto, Japan 1981.

${ }^{33}$ H. Yamada, "Low-temperature 3C-SiC heteroepitaxial film growth on Si by reactive-ion-beam deposition", Journal of Applied Physics, Vol. 65, 2084 (1988).

34 S. Motoyama, N. Morikawa, M. Nasu, S. Kaneda, Journal of Sppled Physics, Vol. 56, 101 (1990).

35 L.B. Rowland, S. Tanaka, R.S. Kern, R.F. Davis, Proceedings $4^{\text {th }}$ Internation Conference Amorphous and Crystalline Silicon Carbide IV, Santa Clara, 84 (1992).

36 T. Yoshinobu, T. Fuyuki, H. Matsunami, "Interface Modification by Hydrocarbon Gas Molecular Beams in Heteroepitaxy of SiC on Si”, Japanese Journal of Applied Physics, Vol. 30, L1086 (1991).

37 T. Yoshinobu, H. Mitsui, Y. Tarui, T. Fuyuki, H. Matsunami, Japanese Journal of Applied Physics, Vol. 31, L1580 (1992).

${ }^{38}$ T. Fuyuki, Y. Tarui, T. Yohsinobu, T Fuyuki, H. Matsunami, Journal of Crystal Growth, Vol. 150, 934 (1995).

39 T. Hatayama, N. Tanaka, T.Fuyuki, H. Matsunami, "Low-Temperature Interface Modification by Hydrocarbon Radicals in Heteroepitaxy of 3C-SiC on Si Clean Surface," Journal of Electronic Materials, Vol. 26, No. 3160 (1997).

${ }^{40}$ C. H. Wu, C. Jacob, X. J. Ning, S. Nishino, P. Pirouz, "Epitaxial Growth of 3C-SiC on Si(111) from Hexamethyldisilane," Journal of Crystal Growth, Vol. 158, No. 4, 480 (1996).

${ }^{41}$ I. Golecki, F. Reidinger, J. Marti, "Single-Crystalline, Epitaxial Cubic SiC Films Grown on (100) $\mathrm{Si}$ at $750^{\circ} \mathrm{C}$ by Chemical Vapor Deposition," Applied Physics Letters, Vol. 60, No. 14, 1703 (1992).

${ }^{42}$ S. Madapura, A. J. Steckl, M. Loboda, "Heteroepitaxial Growth of SiC on Si(100) and (111) by Chemical Vapor Deposition Using Trimethylsilane," Journal of Electrochemical Society, Vol 146, No. 3, 1197 (1999). 
${ }^{43}$ J. H. Boo, S. A. Ustin, W. Ho, "Supersonic Jet Epitaxy of Single Crystalline Cubic SiC Thin Films on Si Substrates from t-Butyldimethylsilane," Thin Solid Films, Vol. 324, 124 (1998).

${ }^{44}$ H. Nakazawa, M. Suemitsu, S. Asami, "Formation of High Quality SiC on Si (100) at $900^{\circ} \mathrm{C}$ using Monomethylsilane Gas-source MBE," Materials Science Forun, Vol. 338-342, 269 (2000).

45 H. Nakawaza, M. Suemitsu, N. Miyamoto, "Gas Source MBE of $\mathrm{SiC} / \mathrm{Si}$ using Monomethylsilane," Thin Solid Films, Vol. 369, 269 (2000).

${ }^{46}$ J.M. Lannon Jr., Nucleation and Growth Studies Relevant do Diamond Thin Film Synthesis, Dissertation, Physics Department, West Virginia University, Fall (1996).

${ }^{47}$ J. S. Gold, Characterization of a Novel Methyl Radical Source and Related Thin Film Growth Studies, Dissertation, Physics Department, West Virginia University, Fall (2000).

${ }^{48}$ K. S. Ziemer, Studies of the Initial Stage of Silicon Carbide Growth on Silicon, Dissertation, Chemical Engineering Department, West Virginia University, Spring (2001).

${ }^{49}$ E.G.Acheson, “On Carborundum”, Chemical News, Vol. 68179 (1893).

${ }^{50}$ J.A.Lely, "Darstellung von Einkristallen von Silicium Carbid und Beherrschung von Art und Menge der eingebauten Verunreinigungen", Ber. Dt. Keram. Ges., Vol. 8229 (1955).

${ }^{51}$ C. Goldberg, C.Z. LeMay, L.F. Wallace, Silicon Carbide, A High Temperature Semiconductor, Ed. J.R. O'connor, J. Smiltens, Pergamon, Oxford, 453 (1960).

${ }^{52}$ Y.M. Tairov, V.F. Tsvetkov, "Investigation of Growth Processes of Ingots of Silicon Carbide Single Crystals", Journal of Crystal Growth, Vol. 43, 208 (1978).

${ }^{53}$ Y.M.Tairov, V.F.Tsvetkov, "General Principles of Growing Large-Size Single Crystals of Various Silicon Carbide Polytypes", Journal of Crystal Growth, Vol. 52, 146 (1981).

${ }^{54}$ St.G. Muller, R.C. Glass, H.M. Hobgood, V.F. Tsvetkov, M. Brady, D. Henshall, J.R. Jenny, D. Malta, C.H. Carter Jr., "The status of SiC bulk growth from an industrial point of view", Journal of Crystal Growth, Vol. 211, No. 1-4, (325) 2000.

${ }^{55}$ H. Jacobson, R. Yakimova, P. Raback, M. Syvajarvi, J. Birch, E. Janzen, "Lateral Enlargement of Silicon Carbide Crystals", Materials Science Forum, Vols. 389-393, 39 (2002).

${ }^{56}$ J.W. Faust, Silicon Carbide, Eds. J.R. O'Connor and J. Smiltens, Oxford Pergamon Publishing, pages 403-419, (1960).

${ }^{57}$ V.L. Jennings, A. Sommer, H.C. Chang, "The Epitaxial Growth of Silicon Carbide”, Journal of the Electrochemical Society, Vol. 113, 728 (1966). 
${ }^{58}$ R.W. Brander, A.L. Boughey, "The Etching of $\alpha$-SiC", British Journal of Applied Physics, Vol. 18, 905 (1967).

${ }^{59}$ E.A. Gulbransen, K.F. Andrew, F.A. Brassart, "Oxidation of Silicon at High Temperatures and Low Pressure Under Flow Conditions and the Vapor Pressure of Silicon”, Journal of the Electrochemical Society Vol. 113, No. 8, 834 (1966).

${ }^{60}$ T. L. Chu, R. B. Campbell, "Chemical Etching of Silicon Carbide with Hydrogen”, Journal of the Electrochemical. Society, Vol. 112, No. 9, 955 (1965).

${ }^{61}$ M. Kumagawa, H. Kuwabara, S. Yamada, "Hydrogen Etch of Silicon Carbide", Japanese Journal of Applied Physics, Vol. 8, No. 4, 421 (1966).

${ }^{62}$ E. Modrak, I. Swiderski, T. Niemyski, "Etching of $\alpha$-SiC Crystals in Hydrogen and Argon Flow", Journal of Crystal Growth, Vol. 22, 181 (1974).

${ }^{63}$ M.S. Saidov, M.A. Medyrov, K.A. Shamuratov, "Hydrogen Etching of the Surface of Silicon Carbide", Fizika i Khimiya Obrabotki Materialov, Vol. 19, No. 4, 136 (1985).

${ }^{64}$ S. Karlsson, N. Nordell, “AFM Study of in-situ Etching of 4H and 6H SiC Substrates”, Materials Science Forum, Vols. 264-268, 363 (1998).

${ }^{65}$ V. Ramachandran, M.F. Brady, A.R. Smith, R.M. Feenstra, D.W. Greve, "Preparation of Atomically Flat Surfaces on Silicon Carbide Using Hydrogen Etching”, Unpublished Report.

${ }^{66}$ Figures based on http://www.chem.qmw.ac.uk/surfaces/scc/scat5 2.htm

${ }^{67}$ A. M. D. Assa'd and M. M. El Gomati, "Backscattering Coefficients for Low Energy Electrons”, Scanning Microscopy, Vol. 12, No. 1, 185 (1988).

${ }^{68}$ Physical Electronics Industries, Inc., Handbook of Auger Electron Spectroscopy, 2nd ed., Eden Prarie, MN (1978).

${ }^{69}$ I. Lindau, W. E. Spicer, "The Probing Depth in Photoemission and Auger-Electron Spectroscopy", Journal of Electron Spectroscopy and Related Phenomena, Vol. 3, No. 5, 409 (1974).

${ }^{70}$ C. J. Powell, “Attenuation Lengths of Low-Energy Electrons in Solids”, Surface Science, Vol. 44, No. 1, 29 (1974).

${ }^{71}$ M. P. Seah, W. A. Dench, "Quantitative Electron Spectroscopy of Surfaces: A Standard Data Base for Electron Inelastic Mean Free Paths in Solids", Surface and Interface Analysis, Vol. 1, No. 1, 2 (1979). 
${ }^{72}$ C. J. Powell, A Jablonski, NIST Electron Inelastic-Mean-Free-Path Database, U.S. Department of Commerce, Technology Administration Standard Reference Data Program, Dec. 2000 .

${ }^{73}$ J. T. Yates, Jr., F. Bozso, W. J. Choyke, L. Muehlhoff, "Studies of SiC Formation on Si(100) by Chemical Vapor Deposition", Journal of Applied Physics, Vol. 57, 771 (1998).

${ }^{74}$ C. S. Fadley, R. Baird, W. Siekhaus, T. Novakov, S. A. L. Bergstrom, "Surface analysis and angular distributions in x-ray photoelectron spectroscopy", Journal of Electron Spectroscopy and Related Phenomena, Vol. 4, No. 3, 93 (1974).

${ }^{75}$ T. S. Sun, D. K. McNamara, J. A. Ahearn, J. M. Chen, B. Ditchek, J. D. Venables, "Interpretation of AES Depth Profiles of Porous Al Anodic Oxides", Applications of Surface Science, Vol. 5, No. 4, 406 (1980).

${ }^{76}$ P. H. Holloway, "Quantitative Auger Electron Spectroscopy EM - Problems and Prospects", Scanning Electron Microscopy (Proceedings of the Annual Scanning Electron Microscope Symposium), No. 1, 361 (1978).

${ }^{77}$ O. K. T. Wu, E. M. Butler, "Auger Signal Intensity Dependence on Surface Area (Roughness)", Journal of Vacuum Science and Technology, Vol. 20, No. 3, 455 (1981).

${ }^{78}$ M. G. Lagally and D. E. Savage, "Quantitative Electron Diffraction from Thin Films," MRS Bulletin 18, 24 (Jan. 1993).

${ }^{79}$ Concepts of drawings taken from Shi, Hongtao. (2002). Exchange Bias and Its Angular Dependence in FexZn1-xF2 / Co Bilayers, Dissertation, West Virginia University, [On-line Abstract]. Available: http://etd.wvu.edu/templates/showETD.cfm?recnum=2732

${ }^{80}$ S. Holloway, J.L. Beeby, "The origins of streaked intensity distributions in reflection high energy electron diffraction”, Journal of Physics C: Solid State Physics, Vol. 11, L247 (1978).

${ }^{81}$ U. Korte, G. Meyer-Ehmsen, "Transmission Features in RHEED from Flat Surfaces", Vacuum, Vol. 41, Iss. 1-3, 343 (1990).

${ }^{82} \mathrm{http}: / /$ www.stoner.leeds.ac.uk/techniques/rheed.htm

${ }^{83}$ G. Binning, C. Quate, C. Gerber, “Atomic Force Microscope,” Physics Review Letters, 56, 930 (1986).

${ }^{84}$ ASME B46.1-1995: "Surface Texture (Surface Roughness, Waviness, and Lay): An American National Standard", American Society of Mechanical Engineers, New York, 1996

${ }^{85}$ D.B. Fenner, D.K. Biegelsen, R.D. Brigans, Journal of Applied Physics, 66, 419 (1989). 
${ }^{86}$ L.S. Hirsch, Preparation of Substrates for Semiconductor Growth Using Atomic Hydrogen, Ph.D. Dissertation, Morgantown, WV, West Virginia University (1998).

${ }^{87} \mathrm{~K}$. Masahara, et al., "Pre-growth treatment of $4 \mathrm{H}-\mathrm{SiC}$ substrates by hydrogen etching at low pressure", Material Science Forum, Vols. 338-342, 1037 (2000).

${ }^{88}$ T. Kimoto, A. Itoh, H. Matsunami, "Step bunching mechanism in chemical vapor deposition on 6H- and 4H-SiC 0001$\} "$, Journal of Applied Physics, Vol 81, 3494 (1997).

89 J.M. Lannon, Jr., J.S. Gold, and C.D. Stinespring, "Evidence for surfactant mediated epitaxy of diamond", Applied Physics Letters, Vol. 73226 (1998).

${ }^{90}$ C.D. Stinespring, J. Wormhoudt, "Surface studies relevant to silicon carbide chemical vapor desposition”, J. Appl. Phys. Vol. 65, 1733 (1989). 


\section{Appendices}

\section{Appendix A: Optical Pyrometer and Emissivity}

The determination of emissivity of $\mathrm{SiC}$ consisted of two different experiments. The first part was of the calibration of the optical pyrometer using a black body heat source. The second part established the relationship between substrate emissivity and substrate temperature.

The optical pyrometer measurements were taken using an Omega OS1562 Fiber Optic Infrared Sensor. A Keithley 2000 Multimeter was used to display the readout. The calibration of optical pyrometer was made using a hollow-sphere type black body heat source. As it heated up to $1000^{\circ} \mathrm{C}$, the temperature and corresponding optical pyrometer measurements were recorded. Since, during the actual growth experiments, the pyrometer measured the substrate temperature across a glass view port window from outside the UHV chamber, an extra step was

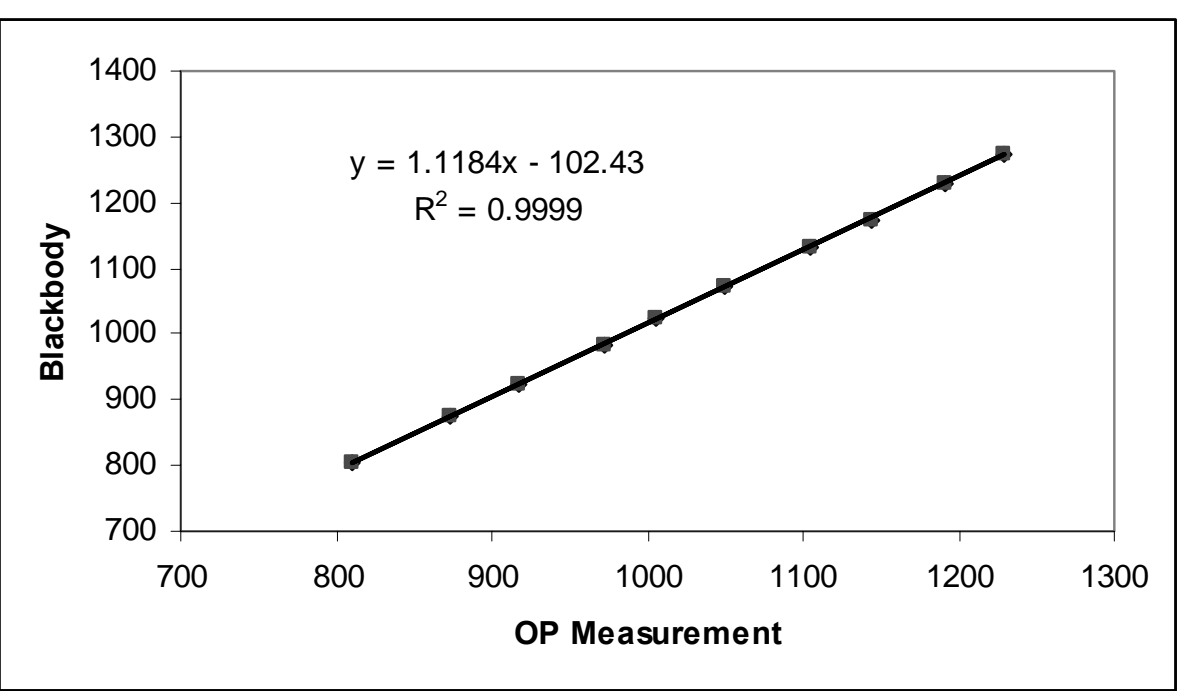

Figure A-1. Black body Optical Pyrometer calibration including losses caused by windows. 
needed to determine the temperature loss with the presence of the window. This calibration curve including the window loss was plotted and shown in Figure A-1. The black body source was believed to have an error of $\pm 3^{\circ} \mathrm{C}$ for the range of temperature here.

The second part of the experiment was done in our high temperature hydrogen furnace. With the water-cooled adapters taken off, the tube was slowed heated up to $1000^{\circ} \mathrm{C}$. A $6 \mathrm{H}-\mathrm{SiC}$ substrate already hydrogen-etched was placed inside the furnace vertically on a carborundum stand, and optical pyrometer measurements were taken on the polished and etched side of the substrate. A B-type thermocouple encased in a ceramic protection tube was inserted into the furnace to directly measure the substrate surface temperature, and the measurement was displayed with an Omega DP462 digital readout. The thermocouple had a manufacturer specified tolerance of $1^{\circ} \mathrm{C}$ for temperature up to $1100^{\circ} \mathrm{C}$. As the substrate was allowed to cooled down slowly (regulated by high temperature furnace heater controller), the temperatures as reported by optical pyrometer and corresponding B-type thermocouple measurements were recorded.

To calculate the relative emissivity (of $\mathrm{SiC}$ to a perfect black body surface), the StefanBoltzmann law was used.

$$
E=s T^{4}
$$

where $\mathrm{E}=$ total energy emitted from a unit area of a surface per second $\mathrm{T}=$ absolute temperature of the surface (i.e. the temperature expressed in $\mathrm{K}$ ) $\mathrm{s}=$ Stefan-Boltzmann constant.

The Stefan-Boltzmann law is valid for any surface that is a perfect absorber of incident radiation - black bodies. Certainly, $\mathrm{SiC}$ substrates were not perfect emitters or absorbers of 


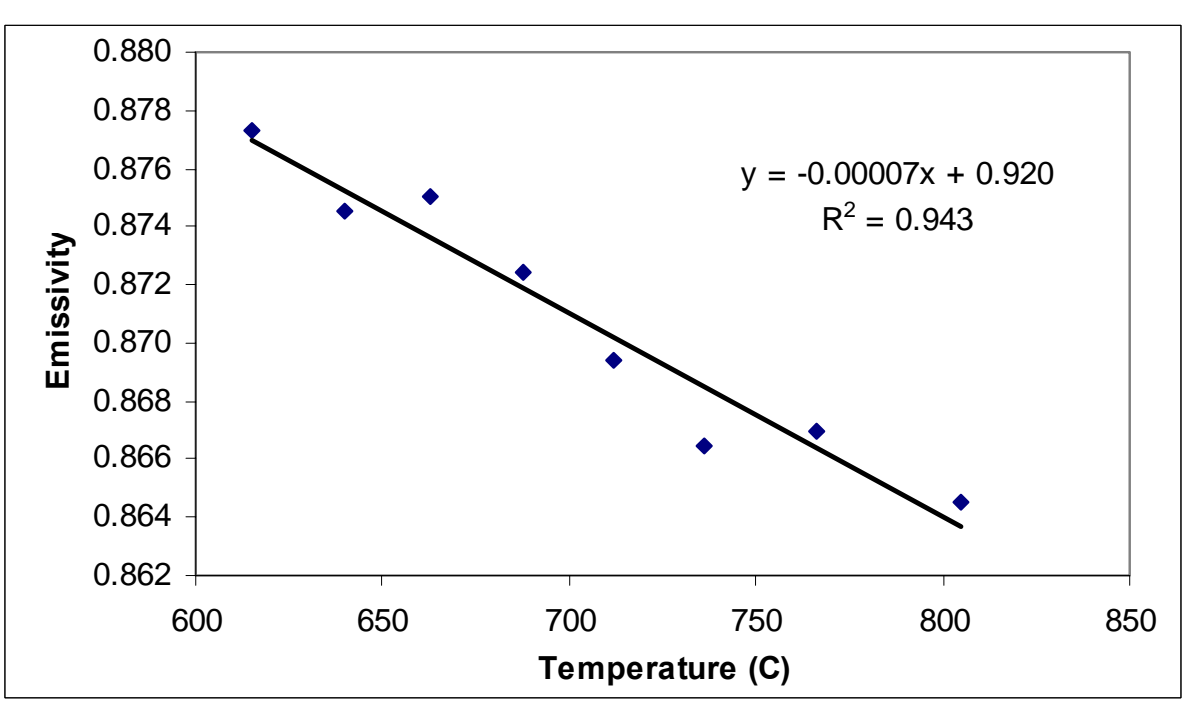

Figure A-2. Emissivity of $6 \mathrm{H}-\mathrm{SiC}$ at substrate temperature range of $600^{\circ} \mathrm{C}$ $800^{\circ} \mathrm{C}$.

radiation (sometime referred to as gray bodies). Emissivity $(\varepsilon)$ was the quantity that represented the ratio that measured energy emitted of a gray body to that of a black body.

$$
\varepsilon=\frac{E_{g}}{E_{b}}=\frac{T_{g}^{4}}{T_{b}^{4}}
$$

Here, $\mathrm{T}_{\mathrm{g}}$ would be the temperature as measured by the optical pyrometer while $\mathrm{T}_{\mathrm{b}}$ would be the actual surface temperature calibrated using a black body. Emissivity of SiC was thus e is dimensionless and should have a value between 0 and 1. Emissivity could then be calculated for individual temperature measurement taken. A plot of emissivity vs. temperature is seen in Figure A-2. 


\section{Appendix B: Relative sensitivity factor of Si-LMM and C-KLL}

Relative sensitivity factor of C-KLL to Si-LMM was needed for calculation of relative composition of the two species in epitaxial growth studies.

It was decided the best reference candidate was that of a heteroepitaxial $\beta$-SiC film grown using MS under high flux and high temperature conditions for 60 minutes. The scan had a good carbidic C-KLL line shape, and Si-LMM line shape also displayed characteristics of SiC bonds. Since the film was thick $(\sim 50 \mathrm{~nm})$ and all the out-diffusion voids had been long sealed, the AES spectra would not be subjected to elemental Si "contamination" from the substrate. In addition, the gas source of methylsilane provided $\mathrm{Si}: \mathrm{C}=1: 1$ ratio, so the surface should not suffer from excess carbon-carbon bonded C-KLL influences. The actual AES spectrum taken is shown in Figure A-3.

The details of Auger relative sensitivity derivations are seen in Section 3.4, and it can be

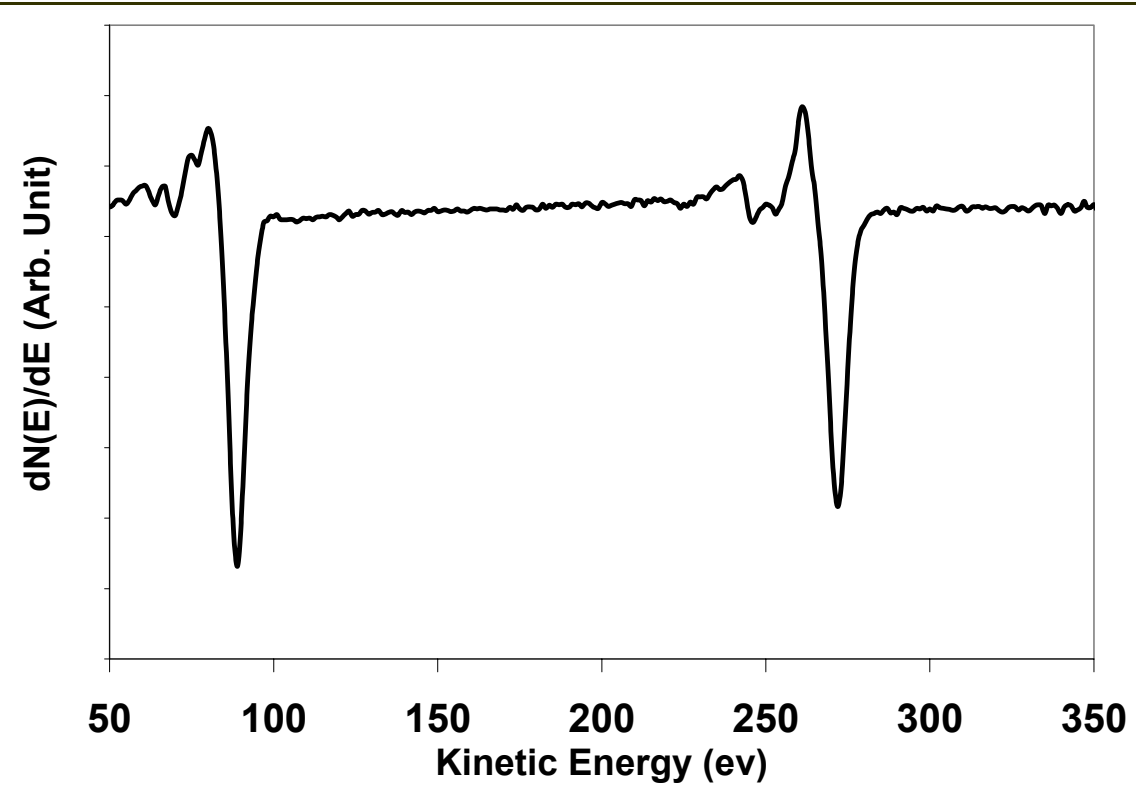

Figure A-3. AES survey spectrum for 60 minutes of heteroepitaxial growth under HFHT conditions using MS. 
summarized as

$$
\frac{I_{i}}{I_{r e f}}=\frac{N_{i} \cdot S_{f-i}}{N_{r e f} \cdot S_{f-r e f}}\left(\frac{A_{i} \cdot \lambda_{i}}{A_{r e f} \cdot \lambda_{r e f}}\right)
$$

Here, $I_{i} / I_{r e f}=$ peak-to-peak intensity ratio as measured by AES;

$$
\begin{aligned}
& \mathrm{N}=\text { the volumetric atomic density }=\left[\text { number of atoms } / \mathrm{cm}^{3}\right] \\
& \mathrm{S}_{\mathrm{f}}=\text { AES sensitivity factor; } \\
& \mathrm{A}=\text { AES scanning area; } \\
& \lambda=\text { Auger electrons mean free paths. }
\end{aligned}
$$

Rearranging the equation for sensitivity factors of C-KLL to Si-LMM yields

$$
\frac{S_{f-i}}{S_{f-r e f}}=\frac{I_{i} N_{r e f}}{I_{r e f} N_{i}}\left(\frac{A_{r e f} \cdot \lambda_{r e f}}{A_{i} \cdot \lambda_{i}}\right)
$$

For our purposes, $\mathrm{N}_{\mathrm{Si}}=\mathrm{N}_{\mathrm{C}}$ due to the assumed stoichiometry of the $\mathrm{SiC}$ film, $\mathrm{A}_{\text {ref }}=\mathrm{A}_{\mathrm{i}}$ since area of AES scan remained the same, and $\lambda_{\mathrm{Si}}=5.2 \AA$ and $\lambda_{\mathrm{C}}=9.16 \AA .^{72}$

Based on the peak-to-peak intensity measurements for the Si-LMM and C-KLL peaks using a $2 \mathrm{keV}$ primary electron beam and a beam current of $1.3 \mu \mathrm{A}$, we determined $\frac{S_{C}}{S_{S i}}=0.68$. 